EFFECTS OF ADULT ATTACHMENT ON HEALTH OUTCOMES AMONG COUPLES

FACING OVARIAN CANCER AND THE MEDIATING ROLE OF DYADIC COPING

by

Cassandra Crangle

Master of Arts, Ryerson University, October 2012

Bachelor of Arts, McGill University, June 2009

A dissertation presented to Ryerson University

in partial fulfillment of the requirements for the degree of

Doctor of Philosophy in the program of Psychology

Toronto, Ontario, Canada, 2017

CCassandra Crangle 2017 


\section{AUTHORS DECLARATION FOR ELECTRONIC SUBMISSION OF A DISSERTATION}

I hereby declare that I am the sole author of this dissertation. This is a true copy of the dissertation, including any required final revisions, as accepted by my examiners.

I authorized Ryerson University to lend this dissertation to other institutions or individuals for the purpose of scholarly research.

I further authorize Ryerson University to reproduce this dissertation by photocopying or by other means, in total or in part, at the request of other institutions or individuals for the purpose of scholarly research.

I understand that my dissertation may be made electronically available to the public. 
Effects of Adult Attachment on Health Outcomes Among Couples Facing Ovarian Cancer and the Mediating Role of Dyadic Coping

Doctor of Philosophy 2017

Cassandra Crangle

Psychology

Ryerson University

\begin{abstract}
Background: Ovarian cancer is commonly diagnosed at a late stage of illness, raising significant challenges to health-related quality of life (QOL). Increasingly, cancer is conceptualized as an interpersonal stressor that significantly impacts patients as well as their spouses. It has been suggested that adult attachment significantly impacts health outcomes by way of dyadic processes. The present study used the actor partner interdependence model (APIM) to examine the dyadic effects of adult attachment and dyadic coping on QOL, and whether dyadic coping mediated the associations between attachment and QOL. Methods: Couples $(\underline{N}=106)$ facing ovarian cancer were recruited from a comprehensive cancer centre in Toronto, ON. Attachment was measured by the Experiences in Close Relationships Scale - Revised, dyadic coping was measured by the Dyadic Coping Inventory, and QOL was measured by the Functional Assessment of Cancer Treatment. APIM analyses evaluated within person and cross-dyadic effects. Role (patients compared with spouses) was examined as a moderator. Results: There were significant within person effects of anxious attachment on physical, social, emotional, and functional QOL. There were also significant within person effects of avoidant attachment on social, emotional, and functional QOL. Positive and negative dyadic coping demonstrated significant within person effects on social and functional QOL. There were no significant cross-
\end{abstract}


dyadic effects nor was there evidence of moderation by role. The within person association between avoidant attachment and social QOL was significantly mediated by positive dyadic coping. The within person association between anxious attachment and social QOL was significantly mediated by positive and negative dyadic coping. Additionally, there was a significant indirect cross-dyadic effect, such that greater anxious attachment reported by one partner was associated with less positive dyadic coping and subsequently less social QOL reported by the other partner. Discussion: Adult attachment and coping as a couple are important considerations in understanding QOL among both patients and spouses. Intervention strategies to address attachment, and the behavioural system associated with attachment, such as dyadic coping, may be useful in addressing impaired individual well-being. Future studies should consider additional dyadic processes that may account for the negative effects of attachment on QOL such as intimacy. 


\section{Acknowledgements}

I would like to sincerely thank my supervisor, Dr. Tae Hart, for your encouragement and guidance. Throughout the past 8 years, I have felt supported, valued, and respected. I am truly grateful for your mentorship and support. I would also like to express my appreciation to Dr. Stephanie Cassin, Dr. Candice Monson, Dr. Faith Donald, and Dr. Karen Kayser for their time, careful consideration, and feedback. Thank you to Lindsey and Mekhala, and our many volunteers, who worked with me to make this project possible. Finally, to Sam, mom, dad, and Becky, thank you for your unconditional love and support - and your patience! 


\section{Table of Contents}

Authors Declaration For Electronic Submission of a Dissertation ............................................ ii

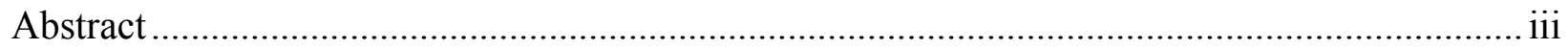

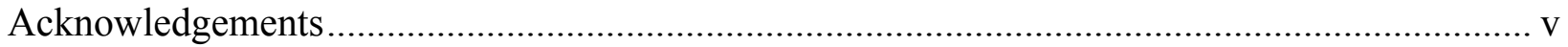

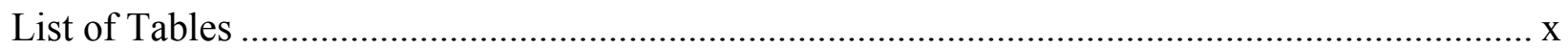

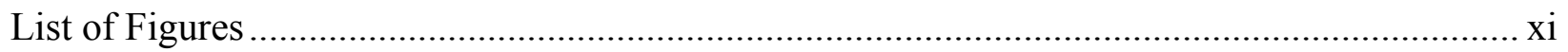

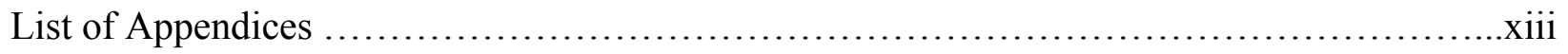

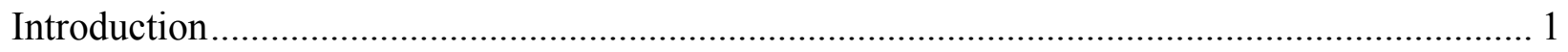

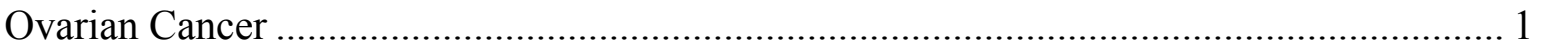

Physical and psychological consequences of ovarian cancer............................. 2

Physical and psychological consequences of ovarian cancer for the partner.......... 3

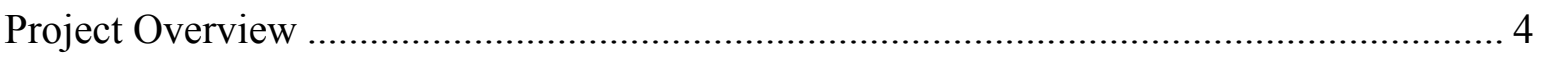

Theoretical Framework for Relationships and Health ................................................ 5

Relationship Orientation: Attachment as a Predictor of Health Outcomes ....................... 8

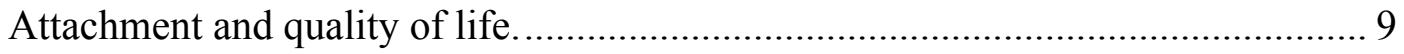

Cross-dyadic effects of attachment on quality of life....................................... 11

Dyadic Processes: Dyadic Coping as a Mediator of the Relationship between Attachment

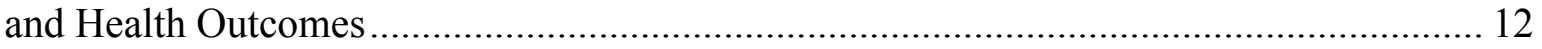

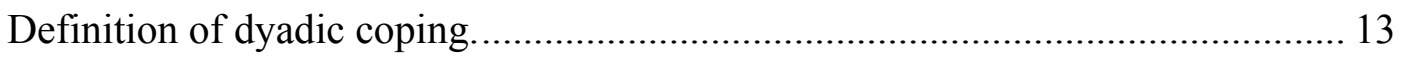

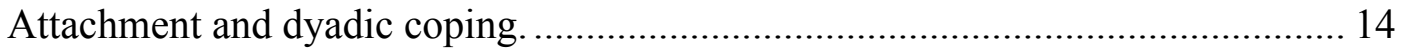

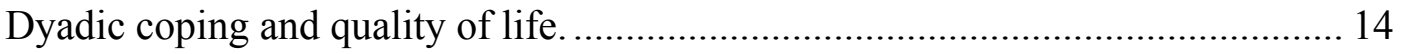

Cross-dyadic effects of dyadic coping on quality of life................................. 15

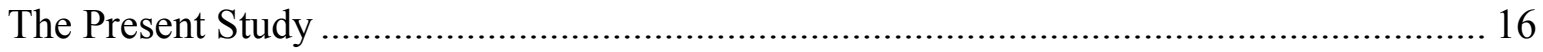


Aims and Hypotheses

Aim 1: To investigate attachment as a predictor of patients' and spouses' healthrelated quality of life.

Aim 2: To investigate dyadic coping as a predictor of patients' and spouses'

quality of life.

Aim 3: To investigate the role of dyadic coping as a mediator between attachment and quality of life.

Method 19

Participants

Procedure

Measures

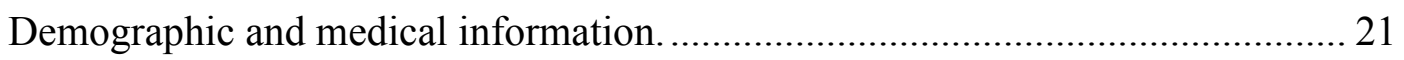

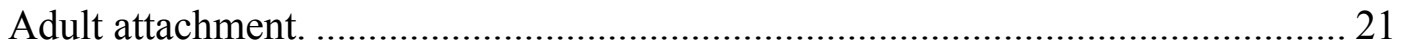

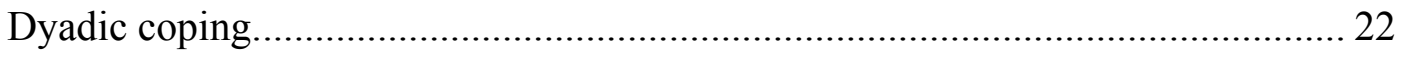

Health-related quality of life. ........................................................................ 22

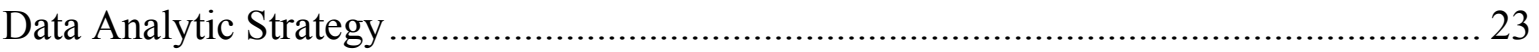

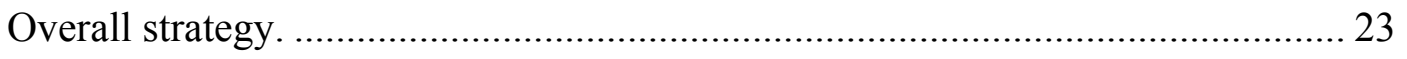

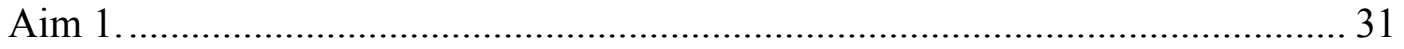

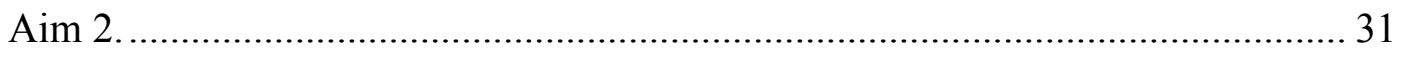

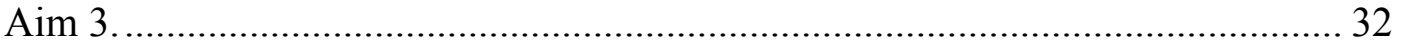

Power Analysis ........................................................................................................... 33

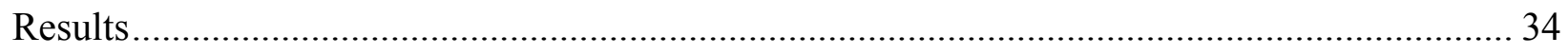

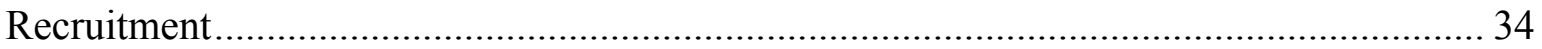


Descriptive Statistics and Preliminary Analyses ............................................................. 34

Aim 1: To Examine the Association between Attachment and Quality of Life .................. 46

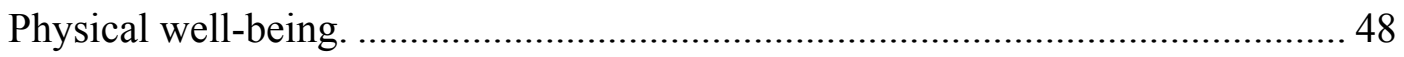

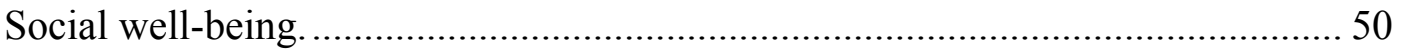

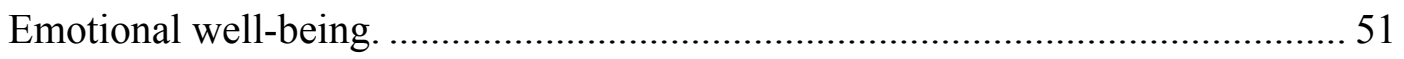

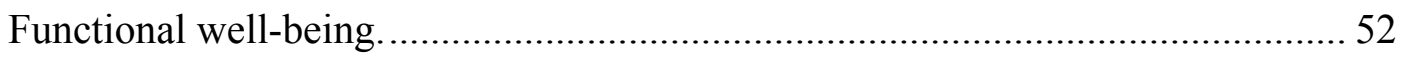

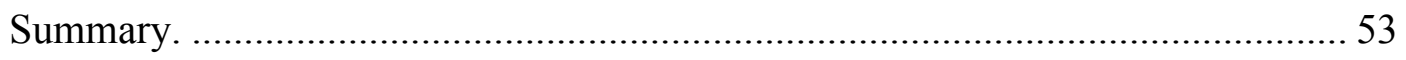

Aim 2: To Examine the Association between Dyadic Coping and Quality of Life ............ 53

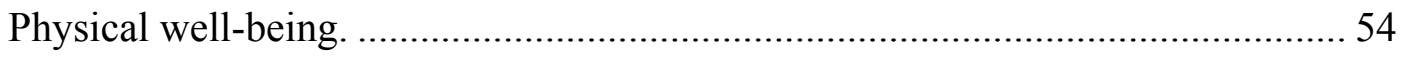

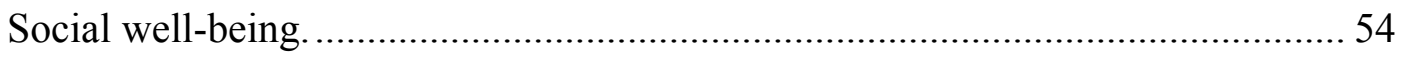

Emotional well-being. ……………….............................................................. 57

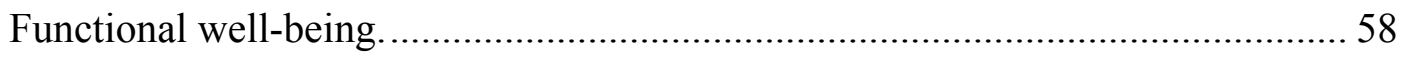

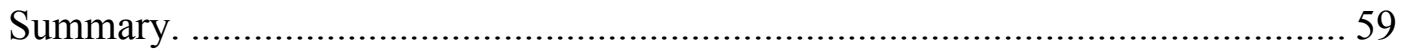

Aim 3: To Examine Dyadic Coping as a Mediator Between Attachment and Quality of Life

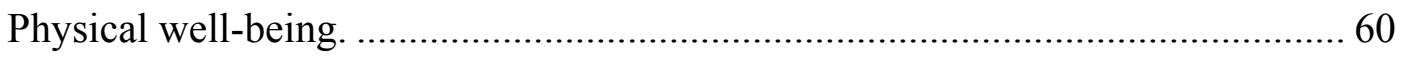

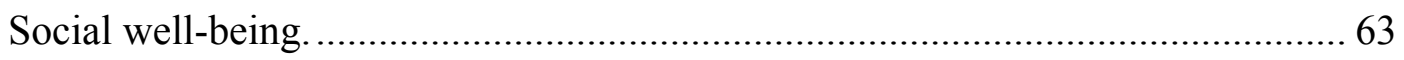

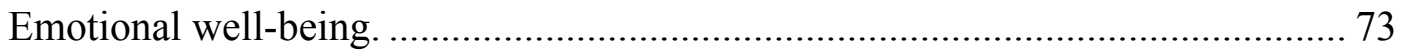

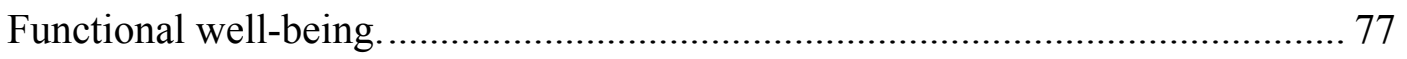

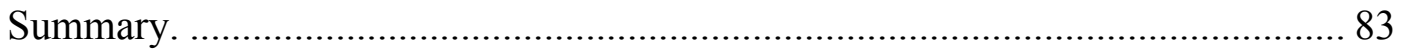

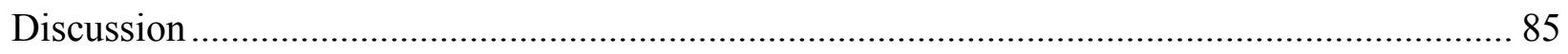

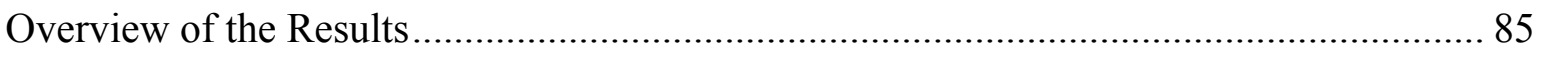

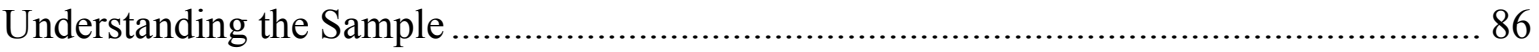




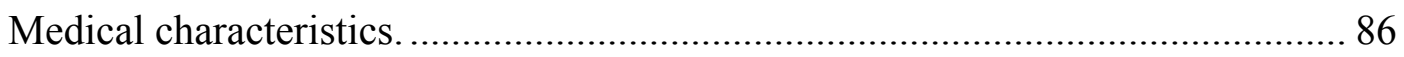

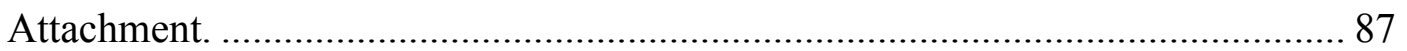

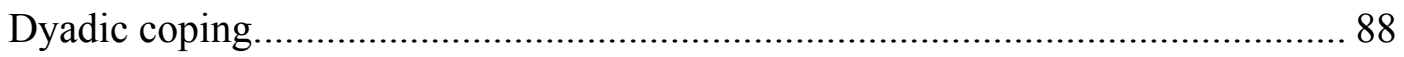

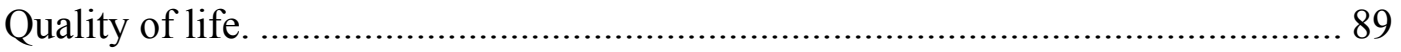

Dyadic Effects of Attachment on Quality of Life........................................................ 90

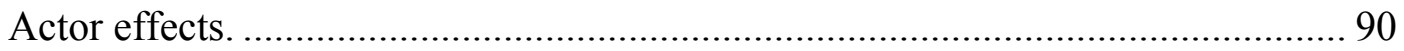

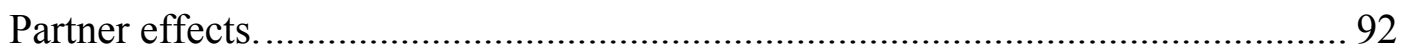

Dyadic Effects of Dyadic Coping on Quality of Life ......................................................... 95

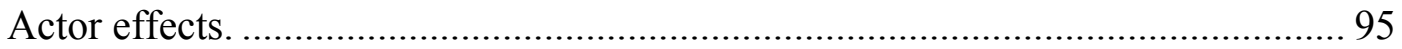

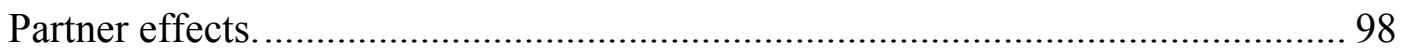

Dyadic Coping as a Mediator Between Attachment and Quality of Life .......................... 100

Actor-actor indirect effects for social well-being.............................................. 100

Partner-actor indirect effect for social well-being.............................................. 101

Mediation of functional well-being................................................................ 102

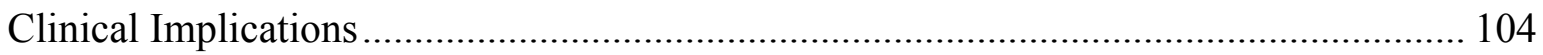

Implications for healthcare professionals........................................................ 104

Clinical implications of the role of attachment. ................................................. 105

Clinical implications of the role of dyadic coping. ............................................. 107

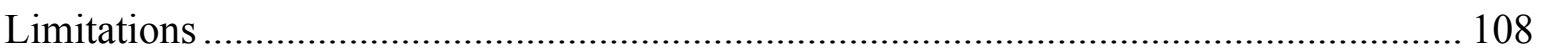

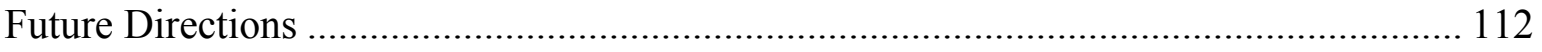

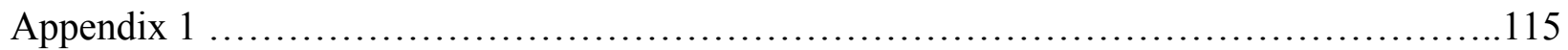

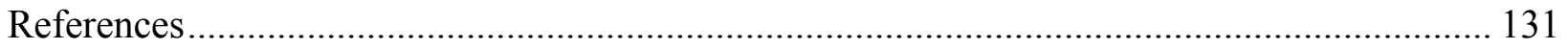




\section{List of Tables}

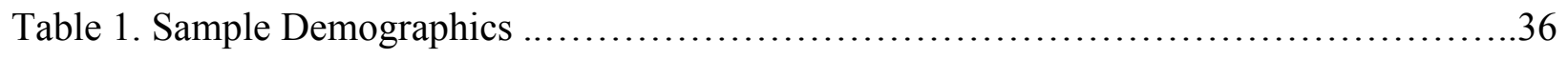

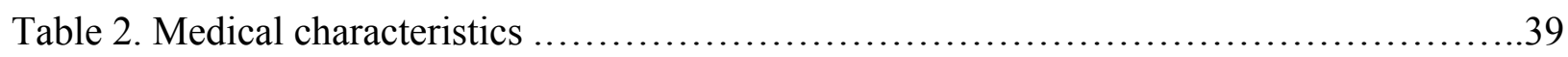

Table 3. Descriptive Statistics of Primary Study Variables $\ldots \ldots \ldots \ldots \ldots \ldots \ldots \ldots \ldots \ldots \ldots \ldots \ldots . \ldots \ldots$

Table 4. Correlations Between Study Variables .......................................45

Table 5. APIM Interaction Model Estimating Effects of Avoidant Attachment on Quality of Life

Table 6. APIM Interaction Model Estimating Effects of Anxious Attachment on Quality of Life

Table 7. APIM Interaction Model Estimating Effects of Positive Dyadic Coping on Quality of

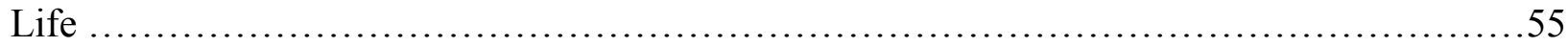

Table 8. APIM Interaction Model Estimating Effects of Negative Dyadic Coping on Quality of

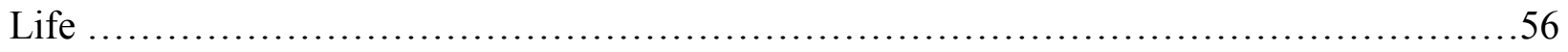




\section{List of Figures}

Figure 1. Reproduction of theoretical framework from Pietromonaco et al. (2013). ..............6

Figure 2. Example APIM interaction model ...........................................24

Figure 3. Example APIM two-intercept model ..........................................26

Figure 4. Example APIMeM interaction model ......................................29

Figure 5. Example APIMeM two-intercept model .......................................30

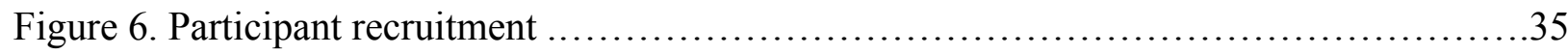

Figure 7. Positive dyadic coping as a mediator for avoidant attachment and physical well-being

Figure 8. Positive dyadic coping as a mediator for anxious attachment and physical well-being

Figure 9. Negative dyadic coping as a mediator for avoidant attachment and physical well-being

Figure 10. Negative dyadic coping as a mediator for anxious attachment and physical well-being

Figure 11. Positive dyadic coping as a mediator for avoidant attachment and social well-being

Figure 12. Positive dyadic coping as a mediator for anxious attachment and social well-being

Figure 13. Negative dyadic coping as a mediator for avoidant attachment and social well-being

Figure 14. Negative dyadic coping as a mediator for anxious attachment and social well-being 
Figure 15. Positive dyadic coping as a mediator for avoidant attachment and emotional wellbeing .... .74

Figure 16. Positive dyadic coping as a mediator for anxious attachment and emotional well-being .75

Figure 17. Negative dyadic coping as a mediator for avoidant attachment and emotional wellbeing .76

Figure 18. Negative dyadic coping as a mediator for anxious attachment and emotional well-

being .78

Figure 19. Positive dyadic coping as a mediator for avoidant attachment and functional wellbeing

Figure 20. Positive dyadic coping as a mediator for anxious attachment and functional well-being

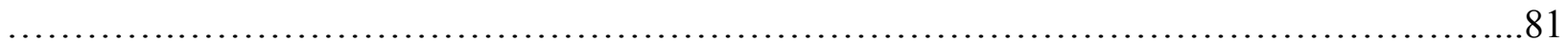

Figure 21. Negative dyadic coping as a mediator for avoidant attachment and functional well-

being

Figure 22. Negative dyadic coping as a mediator for anxious attachment and functional wellbeing .84 


\section{List of Appendices}

Appendix 1. Questionnaires ......................................................... 115 
Ovarian cancer is a prevalent and devastating disease currently facing women and their intimate partners. Among the various physical and psychological challenges facing couples coping with ovarian cancer, quality of life has emerged from the literature as a particularly important health outcome. A theoretical model has been proposed to integrate the fields of health and relationships (Pietromonaco, Uchino, \& Dunkel Schetter, 2013); this model suggests that adult attachment can help explain health outcomes by way of dyadic processes among couples facing chronic illnesses. Parts of this model have been examined, with the results supporting intrapersonal and cross-dyadic associations of attachment with quality of life among patients facing cancer and their partners. Moreover, as dyadic coping has been associated with both attachment and health outcomes, it has emerged as an important dyadic process that may account for these relationships. This study investigated the dyadic effects of attachment and dyadic coping on quality of life, and the extent to which dyadic coping mediated the association between attachment and quality of life, among couples coping with ovarian cancer.

\section{Ovarian Cancer}

Ovarian cancer is the fifth most diagnosed type of cancer among Canadian women, with estimates that 1 in 72 are expected to develop ovarian cancer in their lifetime (Canadian Cancer Society's Advisory Committee on Cancer Statistics, 2016). Ovarian cancer has been referred to as the silent disease; early symptoms such as abdominal pain and bloating, increased urinary urgency, bowel changes, abdominal fullness, and fatigue are common physical symptoms in the general population, which makes early detection difficult (Yawn, Barrette, \& Wollan, 2004). Moreover, early detection methods are insufficient; studies have indicated that screening has no effect on morbidity or mortality (Yawn et al., 2004). 
Given this difficulty with early detection, approximately $60 \%$ of incident cases of ovarian cancer are diagnosed at later stages with stage III or IV tumors (Yawn et al., 2004). The 5-year survival rate in Canada is, on average, $41 \%$ (Canadian Cancer Society's Advisory Committee on Cancer Statistics, 2016). There is an approximate $89 \% 5$-year survival rate for women with stage I tumors, $66 \%$ for women with stage II, $34 \%$ for women with stage III and $18 \%$ for women with IV tumors (Canadian Cancer Society's Advisory Committee on Cancer Statistics, 2016). Moreover, although there are disease-free intervals, up to $80 \%$ of women experience a cancer recurrence, necessitating multiple courses of chemotherapy (Lockwood-Rayermann, 2006). Couples coping with ovarian cancer face an incomparable, chronic stressor that, although typically terminal, is also unpredictable in its timing and course. Despite the poor prognosis, survival rates are improving and researchers have increasingly called for a focus on survivorship concerns (Lockwood-Rayermann, 2006; Roland, Rodriguez, Patterson, \& Trivers, 2013). The psychological and physical well-being of the women and their intimate partners coping with this grave and uncertain diagnosis is of substantial concern.

Physical and psychological consequences of ovarian cancer. Studies have demonstrated the significant physical and emotional effects of ovarian cancer. Women with ovarian cancer report a host of physical symptoms such as pain, fatigue, nausea, and neuropathy, which interfere with activities of daily living and functioning (Lockwood-Rayermann, 2006). Quality of life in ovarian cancer is of particular concern given the high rates of advanced stage diagnoses, aggressive treatment options, and preponderance of cancer recurrence. Surprisingly, studies of long-term gynecological cancer survivors have found no significant differences in overall quality of life compared to healthy controls (Gonçalves, 2010; Rannestad \& Skjeldestad, 2007), as have studies of ovarian cancer patients in particular (Hsieh, Chen, Hsiao, \& Shun, 
2014). These studies have, however, provided evidence of specific deficits that are more prevalent among ovarian cancer patients compared to controls (Gonçalves, 2010). Compared with population norms, ovarian cancer patients have reported specific deficits such as impaired physical, functional, and emotional well-being, and reported greater social well-being (von Gruenigen et al., 2010). It has been suggested that the greater social well-being observed reflects social support provided to individuals coping with such a stressful life event.

\section{Physical and psychological consequences of ovarian cancer for the partner. Cancer} has increasingly been conceptualized as a relational stressor due to the effect it has on both the patient and his or her spouse (Banthia et al., 2003; Berg \& Upchurch, 2007; Kayser, Watson, \& Andrade, 2007). Accordingly, spouses of cancer patients have endorsed significantly greater distress than healthy controls, and comparable or greater levels of distress than the patients themselves (Pietromonaco et al., 2013). Quality of life is an important consideration for spouses of individuals facing cancer. Studies of couples facing cancer have revealed significant "spill over" effects such that the impaired quality of life reported by cancer patients negatively affects the quality of life experienced by their spouse over time (Kershaw et al., 2015; Litzelman, Green, \& Yabroff, 2016).

In a study of couples facing ovarian cancer, descriptively, partners endorsed a lower rating of quality of life compared with both healthy controls and the patients themselves; however, statistical significance was unfortunately not explored as this was not an explicit research question (Arden-Close, Gidron, Bayne, \& Moss-Morris, 2013). Additionally, a 3-year longitudinal study of quality of life among women with ovarian cancer and their spouses described patterns in which the patient's well-being was high and maintained (for spiritual and social well-being) or improved over time (physical and functional well-being) while their 
partners experienced compromised social, emotional, and physical well-being that deteriorated over time (Frost et al., 2012). Analyses for statistical significance were again not completed as different measures were used for patient and spouses.

Studies of other cancer populations reveal poorer general and physical quality of life among partners of lung and breast cancer patients compared with healthy controls (Moreira \& Canavarro, 2013; Mosher, Bakas, \& Champion, 2013; Shor, Grinstein-Cohen, Reinshtein, Liberman, \& Delbar, 2015). Moreover, a study of 373 spouses of cancer patients reported that, although quality of life was more impaired in female spouses than male spouses, only quality of life among male spouses was positively affected by relationship quality and by the patients' quality of life (Bergelt, Koch, \& Petersen, 2008). The authors suggested that female spouses receive support from more sources than male spouses, who are more reliant on their ill spouses for emotional support. The results of this study highlight the particular importance of relationship variables and dyadic effects for female patient-male partner dyads.

\section{Project Overview}

Studies of the effect of cancer on relationships have frequently included only one member of the dyad, such as investigating just caregiver or patient quality of life, and have examined the effects of "relational variables" without assessing both members of the couple (i.e., Feldman \& Broussard, 2005). As elaborated below, the present study seeks to examine quality of life among patients facing ovarian cancer and their spouses from a dyadic perspective, drawing upon a theoretical framework proposed for understanding dyadic processes and health. Specifically, the present study will examine the associations between patient and partner attachment and dyadic coping on quality of life among both members of the dyad. Furthermore, 
the mediating role of dyadic coping will be assessed to determine whether this dyadic process accounts for the effect of attachment on quality of life.

\section{Theoretical Framework for Relationships and Health}

Pietromonaco and colleagues (2013) proposed a theoretical model integrating relationship and biopsychosocial processes with health outcomes in an attempt to address the dearth of theory-driven research in the field of health psychology. The model developed by Pietromonaco and colleagues (2013) is depicted in Figure 1. The model suggests that attachment orientations (i.e., internal working models of relationships) are associated with health outcomes (such as physiology, subjective health, and survival) by way of dyadic processes, including behaviours within the relationship (such as support seeking, caregiving, and conflict) and relational mediators and outcomes (such as dyadic adjustment and commitment; Pietromonaco et al., 2013). For example, a study of 127 couples facing lung cancer found that greater patient insecure attachment was significantly associated with greater patient depression and anxiety and more impaired patient functional and social well-being (Porter et al., 2012).

The model by Pietromonaco and colleagues (2013) proposes that dyadic processes such as support giving and cancer-related communication may account for these associations between attachment and health outcomes. Relationship processes may encourage greater self-care, increase confidence in one's ability to cope, as well as assuage daily physical struggles when ill by providing instrumental support or assistance with chores and other activities of daily living. For example, greater secure attachment may be associated with better communication with one's spouse about symptom related impairments and subsequent needs, which may then be associated with reduced illness-related disturbance. Although not examined as a mediator, a study of ovarian cancer patients demonstrated that the degree to which patients held back sharing 
Partner A

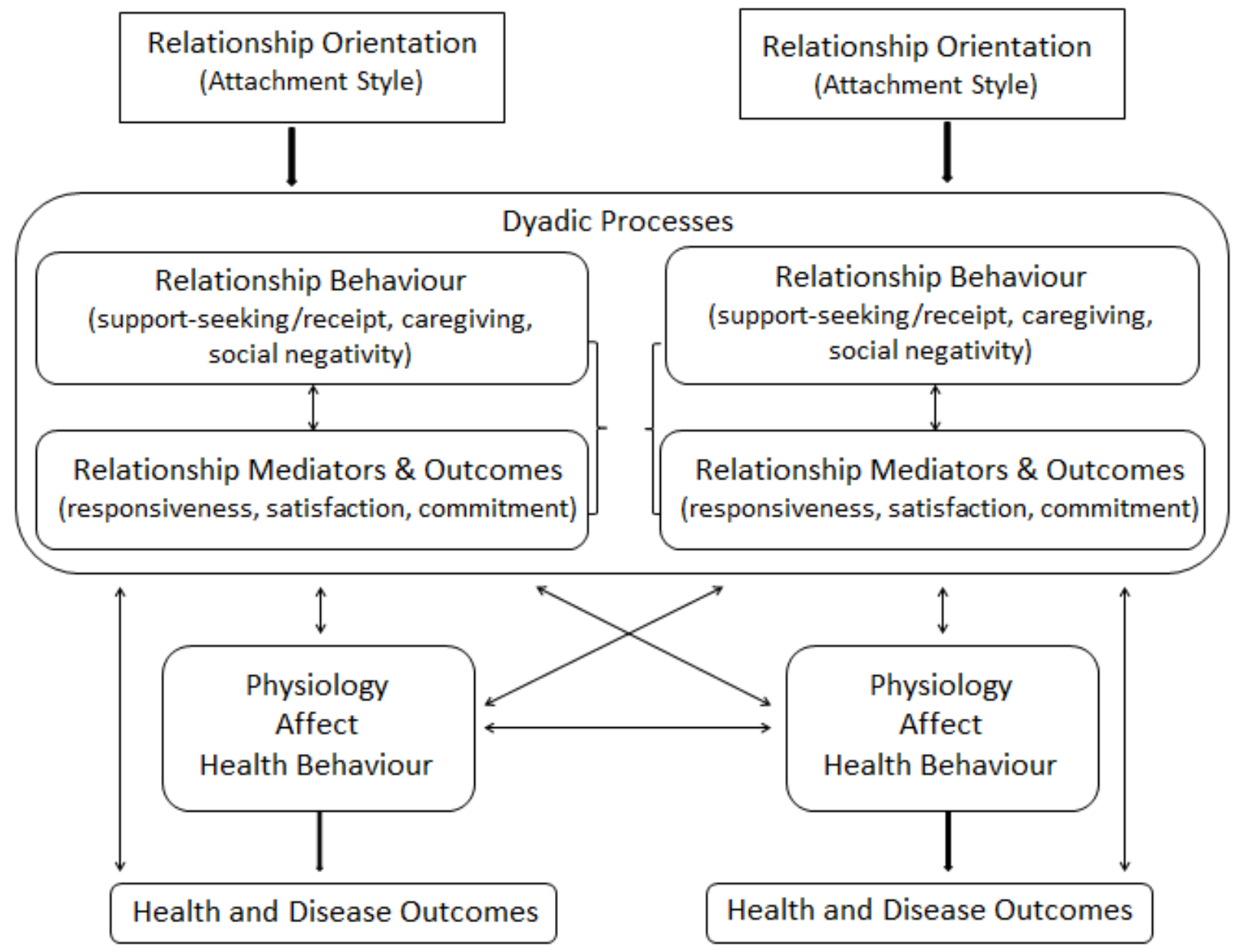

Figure 1. Reproduction of theoretical framework from Pietromonaco et al. (2013). 
concerns about things like finances, relationships, and their physical functioning was negatively associated with their physical and emotional well-being (Manne et al., 2014).

Although the model proposed by Pietromonaco and colleagues (2013) identifies a variety of health-related outcomes such as distal and physiological health outcomes like tumor growth and survival, the present study utilized health-related quality of life to operationalize health outcomes. It is noteworthy that the authors state that the present model is a general one from which researchers can and should derive more specified models (Pietromonaco et al., 2013). The aim of this model is to form a general framework illustrating how to integrate relationship and health research to generate unique, theoretically- and empirically-based research questions and hypotheses. Thus, the flexibility of this model allows and encourages researchers to examine both a subset of constructs specifically identified (i.e. subjective health outcomes), as well as to integrate additional constructs (i.e., dyadic coping as a dyadic process proposed to be a mediator) based on the general framework.

This proposed framework is fundamentally a dyadic model, suggesting dynamic interactions between both the patients suffering from illnesses and their spouses. It explicates the need to evaluate both actor and partner effects; actor effects refer to the association between an individual's own predictors on their own outcomes, whereas partner effects refer to the effects of an individual's predictors on his or her partner's outcomes, or cross-dyadic associations (Kenny, Kashy, \& Cook, 2006). In this way, the term "partner" refers to effects of one member of the dyad on the other dyad member, and not the spouse of the cancer patient. For simplicity, for this present study, partners of cancer patients will be hereafter be referred to as spouses, regardless of marital status, to minimize confusion with the partner effect. Pietromonaco and colleagues (2013) state that, although only a few partner effects are depicted for the aim of simplicity in 
their model, such as the effect of one partner's dyadic processes on the other partner's outcomes, many more partner effects are likely to exist, such as between one individual's attachment and his/her partner's dyadic processes. Indeed, in the aforementioned study of lung cancer patients, greater spouse insecure attachment was significantly associated with greater spouse caregiver strain, depression, anxiety, and anger (i.e., actor effects), as well as more patient pain and impaired patient functional well-being (i.e., partner effects) (Porter et al., 2012). The model suggests that these associations may be mediated by both the patients' and spouses' dyadic processes. For example, both patient and spouse avoidant coping may mediate the effect of spouse insecure attachment on patient pain. Although individual studies may test parts of this model, it highlights the need for studies that directly test mediation by these relational processes (Pietromonaco et al., 2013).

\section{Relationship Orientation: Attachment as a Predictor of Health Outcomes}

One of the key elements of the model by Pietromonaco and colleagues (2013) is that it involves attachment style as an originating construct, a line of research that has garnered significant empirical support over the past 25 years within the fields of both relationship research and health psychology. Attachment refers to working models of relationships with close others, internalized from early relationships with primary caregivers (Cassidy \& Shaver, 2008; Ciechanowski, Walker, Katon, \& Russo, 2002; Pietromonaco et al., 2013; Schmidt, Nachtigall, Wuethrich-Martone, \& Strauss, 2002). Based on these early interactions, individuals develop assumptions and expectations regarding strategies for handling stressors, the viability of support seeking, and the reliability and dependability of others (Cassidy \& Shaver, 2008; Schmidt et al., 2002). Adult attachment typically concerns one's spouse as the primary attachment figure, and refers to an internal framework that guides processes such as expectations of relationships and of 
close others, attributions about self and other behaviour, care-seeking and care-providing behaviour, and views of self and others (Doherty \& Feeney, 2004).

Attachment has been conceptualized both categorically and dimensionally. Two continuous dimensions of attachment have been identified: avoidant and anxious attachment (Ravitz, Maunder, Hunter, Sthankiya, \& Lancee, 2010). Individuals high in avoidant attachment are characterized by a positive view of themselves and a negative view of others (Cassidy \& Shaver, 2008). These individuals tend to use deactivating coping strategies such as minimizing their dependence on others, being overly self-reliant, and avoidance or minimizing of distressrelated cues. Individuals high in anxious attachment are characterized by a negative view of themselves and a positive view of others (Cassidy \& Shaver, 2008). These individuals tend to use hyperactivating coping strategies such intensified signals of distress, excessive support seeking, and seeking reassurance from others.

The model proposed by Pietromonaco and colleagues (2013) suggests that greater anxious or greater avoidant attachment, generally referred to as greater insecure attachment, is significantly predictive of negative health outcomes including negative affect, poorer health behaviours, and decreased subjective health. This model can be applied to various health concerns, including cancer.

Attachment and quality of life. Attachment has been shown to have a significant effect on quality of life in populations facing cancer and other chronic illnesses (Martin, Vosvick, \& Riggs, 2012). For example, among couples facing lung cancer, patients high in insecure attachment reported significantly more impaired quality of life; specifically, patients high in avoidant attachment reported lower social and functional well-being, and patients high in anxious attachment reported more impaired social well-being (Porter et al., 2012). Additionally, 
a study of breast cancer patients examined the effects of attachment on the physical, psychological, and personal relations domains of quality of life (Ávila, Brandão, Teixeira, Coimbra, \& Matos, 2015). Greater anxious and avoidant attachment were associated with worse psychological and personal relations-related quality of life (Ávila et al., 2015).

To date, only a single study has examined attachment and quality of life in gynecological cancer patients (Hsieh et al., 2014). Eighty-five ovarian, cervical, and endometrial cancer survivors and 85 age-matched controls were assessed at a single time-point. Among the gynecologic cancer patients, attachment anxiety was significantly associated with physical quality of life after accounting for sociodemographic and general health variables; in fact, attachment anxiety was found to be the strongest predictor for worse physical quality of life among cancer survivors. In contrast, among the healthy controls, neither attachment anxiety nor avoidance were significantly associated with physical quality of life (Hsieh et al., 2014). The authors suggested that this association between attachment anxiety and worse physical quality of life may be due to intensified perceptions of life stress, greater use of maladaptive coping strategies, or poorer adherence to a healthy lifestyle (such as diet and exercise). Although they did not explicitly address why attachment is associated with physical quality of life among cancer patients and not controls, this result is in line with attachment theory as it is proposed that these internal working models are activated by stress (Cassidy \& Shaver, 2008).

Despite evidence of impaired quality of life, studies have not examined the associations between attachment and quality of life among spouses of patients facing cancer. Attachment among cancer caregivers has been associated with other outcomes, such as caregiving styles (Braun et al., 2012; Kim \& Carver, 2007) and psychological distress (Porter et al., 2012) with the results supporting the beneficial role of a more secure attachment style. For example, among 
spouses of lung cancer patients, greater avoidant attachment was significantly associated with impaired marital quality, greater caregiver strain, increased depression, and more anger, while greater anxious attachment was associated with greater anxiety (Porter et al., 2012).

Cross-dyadic effects of attachment on quality of life. Evidence of cross-dyadic effects of attachment on quality of life is limited. In a study of couples facing lung cancer, greater spouse avoidant attachment was significantly predictive of more patient reported pain and more patient impaired quality of life, specifically functional well-being (Porter et al., 2012). The reverse, that is, the effect of patient reported attachment on spouse quality of life, was not investigated.

Further evidence of the importance of cross-dyadic effects on health outcomes is found in studies of cross-dyadic effects of other psychosocial variables such as psychological distress or illness-appraisals on health-related quality of life (Kim et al., 2008; Merz et al., 2011; Wu, Mohamed, Winkel, \& Diefenbach, 2013). For example, among couples facing breast cancer, dyadic data analysis demonstrated significant cross-dyadic effects of psychological distress on quality of life (Kim et al., 2008). For both patients and spouses, greater psychological distress reported by one member of the couple was significantly associated with more impaired quality of life reported by the other member (Kim et al., 2008). Additionally, among women with breast cancer and their caregivers (husbands, daughters, friends, or other relatives), there were significant cross-dyadic effects on quality of life indicators (Segrin et al., 2005). That is, there was a positive effect of patient stress on caregiver depression, positive effect of patient negative affect on caregiver depression, and negative effect of caregiver positive affect on patient depression (Segrin et al., 2005). 
In sum, there is preliminary evidence of the importance of attachment to quality of life among ovarian cancer patients, however more data are needed to validate this association. Additionally further research is needed on the effects of attachment on health-related quality of life among spouses of ovarian cancer patients. Moreover, studies that examine cross-dyadic effects within couples for each member of the dyad are necessary.

\section{Dyadic Processes: Dyadic Coping as a Mediator of the Relationship between Attachment and Health Outcomes}

As seen in Figure 1, these associations described above between attachment and health outcomes, specifically quality of life, are predicted to be mediated by maladaptive interpersonal processes such as decreased social support, greater social negativity, and impaired relationship quality. One dyadic process that is particularly important for couples facing chronic illness is dyadic coping (Berg \& Upchurch, 2007). A study of coping among couples facing cancer highlighted the frequent theme in which couples discussed cancer stress as "ours," conceptualizing cancer to be a "we-disease" (Kayser et al., 2007). Greater dyadic coping has been consistently associated with better relationship adjustment among the general population, as well as samples of individuals coping with cancer (Badr, Carmack, Kashy, Cristofanilli, \& Revenson, 2010; Bodenmann, 2005; Hagedoorn, Buunk, Kuijer, Wobbes, \& Sanderman, 2000; Traa, De Vries, Bodenmann, \& Den Oudsten, 2015).

In a study of couples diagnosed with metastatic breast cancer, patients and spouses experienced significantly greater psychological distress when they perceived their spouses as being more unsupportive and when they reported using more negative dyadic coping (Badr et al., 2010). Additionally, among couples facing prostate cancer, more collaboration between spouses in daily decision making was associated with more positive and less negative daily affect (Berg 
et al., 2008). Despite the benefits associated with dyadic coping, it may be particularly difficult within the circumstances of a chronic stressor; a study of couples facing chronic obstructive pulmonary disease revealed more negative and less positive dyadic coping compared to healthy controls (Meier et al., 2012).

Definition of dyadic coping. Dyadic coping is a complex construct that has been operationalized in various ways, including individual efforts to cope within the context of a marriage, congruence or discrepancy in coping strategies between dyad members, and coping that is focused on bettering the functioning of the relationship (Bodenmann, 2005). However, Bodenmann's conceptualization of dyadic coping is that of a transactional process in which both members of the dyad are involved, communicate stress to one another, and cope together as a unit (Bodenmann, 2005; Bodenmann, Meuwly, \& Kayser, 2011). Stress that is experienced by one or both members of the dyad is communicated to the other spouse and subsequently can trigger joint coping.

Dyadic coping can occur in a variety of ways. For example, spouses can assist through activities such as aiding in instrumental activities, providing empathy, and communicating their belief in their spouses' capabilities (Bodenmann, 2005). Additionally, dyadic coping can include one spouse taking over the responsibilities of the other to reduce the stress they are experiencing as a couple (Bodenmann, 2005). Dyadic coping may also involve both spouses taking part in the coping equally through joint problem solving, seeking information together, or mutual emotionfocused coping (Bodenmann, 2005). There are also negative forms of dyadic coping, such as hostile interactions (like distancing or open disinterest), one spouse supporting the other unwillingly, and spouses providing insincere support to one another (Bodenmann, 2005). 
Attachment and dyadic coping. Research has supported the association between attachment and dyadic coping. For example, among healthy couples, there was a negative association between anxious and avoidant attachment and positive dyadic coping (Fuenfhausen \& Cashwell, 2013). In fact, positive dyadic coping significantly mediated the association between insecure attachment and impaired dyadic adjustment (Fuenfhausen \& Cashwell, 2013). However, more research is needed on the effects of attachment on dyadic coping in the context of a chronic illness. Indeed, attachment theory proposes that the attachment framework is activated with stress and uncertainty (Cassidy \& Shaver, 2008; Hunter \& Maunder, 2001), suggesting that attachment is particularly relevant to individuals coping with chronic illness, and may play a relatively greater role in understanding dyadic coping in these circumstances.

Dyadic coping and quality of life. Dyadic coping, broadly defined by constructs such as social support and communication within the dyad, has been significantly associated with improved health related quality of life. For example, among ovarian cancer patients, emotional support seeking, and not instrumental support seeking, was found to be significantly associated with better overall quality of life, social well-being, and functional well-being (Hill, 2016). Social support and dyadic communication were assessed among 134 prostate cancer patients and their spouses; open dyadic communication was operationalized as measuring the extent to which each dyad member perceives they share cancer-related issues with one another (Song et al., 2011). For both patients and spouses, greater social support and communication positively predicted health-related quality of life. Among women with cancer, more mutuality in their relationships (empathic and supportive expression of feelings), less protective buffering (previously defined as excluding one's spouse from the coping process to protect them from distressing situations), and less self-silencing (the belief that women must be self-sacrificing and 
silence themselves to maintain their relationships) were all significantly associated with better quality of life (Kayser \& Sormanti, 2002).

Bodenmann's systemic conceptualization of dyadic coping has been used to examine health-related outcomes. Among couples facing breast cancer, there were significant main effects of negative, delegated (a form of positive dyadic coping when one partner takes over the responsibilities of the other to reduce stress), and common (when both partner participate in the coping process more or less equally) dyadic coping on depression for both patients and spouses (Rottmann et al., 2015). Similarly, a study of couples facing prostate cancer revealed significant main effects of negative dyadic coping on anxiety and depression (Regan et al., 2014). A study of spouses of breast cancer patients reported that greater hostile dyadic coping, such as disparagement, distancing, and open disinterest, was significantly associated with greater illness intrusiveness, defined as illness-induced lifestyle disruptions to health, work, finances, family relations, and sex (Feldman \& Broussard, 2006). Although there is initial evidence, further research is needed within ovarian cancer patients and their spouses that specifically examines Bodenmann's systemic dyadic coping as well as research examining quality of life as an outcome. Moreover, studies that investigate the mediated role of dyadic coping between attachment and quality of life are necessary.

Cross-dyadic effects of dyadic coping on quality of life. There is limited research examining the cross-dyadic effects of dyadic coping on quality of life. Studies have demonstrated cross-dyadic effects of Bodenmann's conceptualization of dyadic coping and health-related outcomes. In a study of couples facing breast cancer, spouse negative dyadic coping was associated with greater patient depression, and patient positive dyadic coping was associated with less spouse depression (Rottmann et al., 2015). Consistently, a study of prostate 
cancer couples found significant negative cross-dyadic effects of supportive dyadic coping and both anxiety and depression (Regan et al., 2014). Additionally, among spouses of breast cancer patients, patient physical functioning was positively associated with spouse positive dyadic coping and negatively associated with spouse negative dyadic coping (Feldman \& Broussard, 2006). These studies, however, have yet to examine quality of life.

The association between dyadic coping and quality of life has been examined among chronic obstructive pulmonary disease patients and their partners. A longitudinal study examined dyadic effects of dyadic coping, using Bodenmann's conceptualization, on quality of life at baseline and at 3-months follow-up (Vaske et al., 2015). Two cross-dyadic effects emerged, greater patient delegated coping at baseline predicted greater partner quality of life at follow-up, and greater partner communication of stress at baseline predicted greater patient quality of life at follow-up (Vaske et al., 2015). Further studies are needed that utilize Bodenmann's conceptualization of dyadic coping to examine its cross-dyadic effect on quality of life, particularly within the ovarian cancer population.

\section{The Present Study}

The present study addresses the limitations of the existing literature by evaluating research questions generated from the framework proposed by Pietromonaco and colleagues (2013) within an ovarian cancer population, and examining both members of the dyad. This study additionally examines Bodenmann's systemic conceptualization of dyadic coping (Bodenmann, 1997; Bodenmann et al., 2011), specifically positive and negative dyadic coping, to address concerns about the wide variety of ways that dyadic coping is conceptualized in the literature. The present study examines the effects of both attachment and dyadic coping on quality of life, specifically each of physical, social, emotional, and functional well-being, using 
dyadic data analysis to examine both intrapersonal and cross-dyadic effects. Additionally, consistent with the model that suggests dyadic processes mediate the association between attachment and health outcomes, dyadic coping was examined as a mediator of the association between attachment and quality of life.

\section{Aims and Hypotheses}

\section{Aim 1: To investigate attachment as a predictor of patients' and spouses' health-} related quality of life.

Hypothesis 1. It was hypothesized that one's own greater avoidant and anxious attachment would be negatively associated with one's own physical, social, emotional, and functional well-being ("actor effects").

Hypothesis 2. It was hypothesized that one's own greater avoidant and anxious attachment would be negatively associated with one's partner's physical, social, emotional, and functional well-being ("partner effects").

Exploratory Analysis 1. This study examined whether the actor and partner effects of attachment on health-related quality of life were significantly moderated by role, to determine whether these associations differed in magnitude for patients versus spouses.

\section{Aim 2: To investigate dyadic coping as a predictor of patients' and spouses' quality}

of life.

Hypothesis 3. It was hypothesized that one's own greater positive dyadic coping and less negative dyadic coping would be associated with one's own greater physical, social, emotional, and functional well-being (“actor effects"). 
Hypothesis 4. It was hypothesized that one's own greater positive dyadic coping and less negative dyadic coping would be associated with one's partner's greater physical, social, emotional, and functional well-being ("partner effects").

Exploratory Analysis 2. This study examined whether the actor and partner effects of dyadic coping on quality of life were significantly moderated by role, to determine whether these associations differed in magnitude for patients versus spouses.

\section{Aim 3: To investigate the role of dyadic coping as a mediator between attachment}

\section{and quality of life.}

Hypothesis 5. It was hypothesized that the associations between one's own greater insecure attachment (i.e., anxious and avoidant attachment) and one's own lower health-related quality of life ("actor effect") and one's partner's lower health-related quality of life ("partner effect") would be significantly mediated by one's own and one's partner's less positive and more negative dyadic coping. 


\section{Method}

\section{Participants}

Participants were recruited from Princess Margaret Hospital's medical oncology, radiation and surgical oncology clinics for women with gynecologic cancers. Study staff approached patients in clinic. Study staff received a list of patients attending clinic each day and screened their electronic medical records to determine eligibility based on diagnosis. Study staff received permission from the patients' physician or another member of their healthcare team to approach the patient and then provided a brief introduction to the study, answered questions, and inquired about their interest in participating. If time permitted, verbal consent was obtained in the clinic. If the participant was interested but unable to provide consent in the clinic, study staff obtained the patients' contact information and completed verbal consent over the phone.

Informed consent for spouses took place using the same process; if they were present in clinic and time permitted, verbal consent was obtained in the clinic, otherwise study staff received permission to follow-up by phone. If the spouse was not present in clinic, study staff asked the patient to provide her spouse with an information sheet and to inquire about whether s/he would be interested; study staff then followed up with the patient to evaluate partner interest and received permission from the patient to contact her spouse by phone to complete the consent process. Patients and spouses each received an information letter with an overview of the study, as well as a written copy of the consent form.

Inclusion criteria for this study were if patients had received a diagnosis of invasive ovarian cancer - as opposed to a diagnosis of a borderline tumor, which does not grow into the supportive tissue of the ovary, were able to read and speak English, were over the age of 18, were able to provide informed consent, had been in a relationship for at least 3 months, and had a 
partner who also agreed to participate. Patients were excluded if medical staff recommended that they not participate in the study or if their partner did not complete the study. Spouses were eligible for this study if they were in a relationship with an individual who had been diagnosed with ovarian cancer who agreed to participate in the study, could read and speak English, were over the age of 18 , and were able to provide informed consent. Spouses were excluded from the study if the ovarian cancer patient decided not to participate.

\section{Procedure}

Interested participants provided verbal consent in clinic or over the phone according to preference or available time in clinic. The individual obtaining consent followed a consent script and initialed and signed consent forms to indicate that all relevant information had been provided. Participants were invited to ask questions or request clarification as needed. When it was deemed that the participant was clear on the study purpose and participation, verbal consent was obtained by explicitly asking the participant, "Do you consent to participate in this study?" Study staff provided participants with an information letter and a copy of the written consent form for their own records. These documents were provided in clinic when possible or were emailed or mailed to participants according to preference.

After providing informed consent, each couple was assigned a study ID to maintain confidentiality. Each participant completed a set of questionnaires individually that were completed online, or participants were able to request a paper-copy version. For the online questionnaire, participants were emailed a link to a web-address and given their study ID number to enter into the survey. Participants who chose to complete a paper-based version were mailed the questionnaire package with their study ID number, along with a pre-stamped and addressed return envelope. Participants were told that at any time during the survey they could change their 
mind and choose to stop or refuse to answer any item. For both online and paper questionnaires, they were also able to take a break and return to the survey at a later time. The surveys took approximately 60 minutes to complete. Patients and spouses each received a $\$ 20$ gift card for their participation.

\section{Measures}

For the purpose of the present study, measures assessing adult attachment, dyadic coping, and quality of life were used. Additionally, demographic and medically-relevant information was collected.

Demographic and medical information. Demographic and medical information were collected via self-report. Demographic variables collected from both patients and spouses included age, relationship duration, employment, education, income, and ethnicity. Medical variables that were collected from patients included stage of diagnosis, current treatment, past treatment, treatment progress, time since diagnosis, and the presence of a mutation of the BRCA gene. Mutation of the BRCA1 or BRCA2 gene is hereditary and results in an increased risk of several types of cancer, particularly ovarian and breast cancer (Stoppa-Lyonnet, 2016). Demographic and medical variables were examined as covariates, as elaborated in the data analysis section.

Adult attachment. The Experiences in Close Relationships Scale - Revised (ECR-R; Fraley, Waller, \& Brennan, 2000) was used to assess adult attachment among both patients and spouses. The ECR-R is an 18 item measure in which responses were rated to produce two continuous dimensions: avoidant and anxious attachment. Participants were asked to rate the extent to which they agreed with each statement on a Likert scale ranging from 1 (disagree strongly) to 7 (agree strongly). Higher scores represent greater insecure attachment. Cronbach's 
alpha coefficients of anxious attachment were 0.86 for patients and 0.70 for spouses. Cronbach's alpha coefficients of avoidant attachment were 0.81 for patients and 0.81 for spouses.

Dyadic coping. The Dyadic Coping Inventory (DCI; Bodenmann, 1997; Ledermann et al., 2010) was used to assess dyadic coping among both patients and spouses. The DCI is a 37item measure; participants were asked to rate the extent to which they engaged in a variety of coping behaviours and the extent their partners engaged in the same behaviours. Responses were rated on a 5 point scale, ranging from "very rarely" to "very often." Item examples include: "I ask my partner to do things for me when I have too much to do," "my partner shows empathy and understanding to me," "when my partner feels he/she has too much to do, I help him/her out," and "my partner does not take my stress seriously." For the purpose of the present study, the positive dyadic coping and negative dyadic coping aggregate scales were examined. Cronbach's alpha for negative dyadic coping was 0.76 for patients and 0.73 for spouses. Cronbach's alpha for positive dyadic coping was 0.92 for patients and 0.89 for spouses.

Health-related quality of life. Quality of life was measured with the Functional Assessment of Cancer Therapy - Ovarian (FACT-O) for patients and the FACT - general population (FACT-GP; Brucker, Yost, Cashy, Webster, \& Cella, 2005) for spouses. Both the FACT-O and FACT-GP were comprised of the following subscales, in addition to a total score: physical well-being, social well-being, emotional well-being, and functional well-being. For the purpose of the present study, the physical, social, emotional, and functional well-being scales were examined. Cronbach's alpha coefficients for physical well-being was 0.89 for patients and 0.62 for spouses. Cronbach's alpha coefficients for social well-being was 0.83 for patients and 0.79 for spouses. Cronbach's alpha coefficients for emotional well-being was 0.85 for patients 
and 0.78 for spouses. Cronbach's alpha coefficients for functional well-being was 0.86 for patients and 0.88 for spouses.

\section{Data Analytic Strategy}

Overall strategy. Statistically, dyadic data violate the fundamental assumption of independence of standard statistical methods that are meant for individuals. Dyadic data are inherently non-independent as the responses from each dyad member are related to the other members' response in a meaningful way; members of a dyad are likely to be more similar or more different to one another than people outside of the dyad. Using standard statistical methods with dyadic data results in biased standard errors and loss of degrees of freedom, which tend to negatively influence tests of significance (Kenny et al., 2006).

The actor-partner interdependence model (APIM) developed by Kenny and colleagues (2006) treats the dyad as the unit of analysis to account for non-independence. An example of the basic APIM model, referred to as the interaction model, is displayed in Figure 2. The effects represented by $a$ are called actor effects; actor effects are intrapersonal effects, or the influence of an individual's own predictor variable on his or her own outcome variable. For example, the actor effect refers to the effect of an individual's own attachment on one's own quality of life, or the effect of actor attachment on actor quality of life. The effects represented by $p$ are called partner effects; partner effects are cross-dyadic effects, or the influence of an individual's spouse's predictors on an individual's own outcome variable. For example, the partner effect refers to the effect of an individual's spouse's attachment on an individual's own quality of life, or the effect of partner attachment on actor quality of life. Noteworthy, the term partner refers to the other member of the dyad rather than the individual of the dyad without cancer. Thus each member of the dyad is both an actor and a partner. To reduce confusion, partners of cancer 


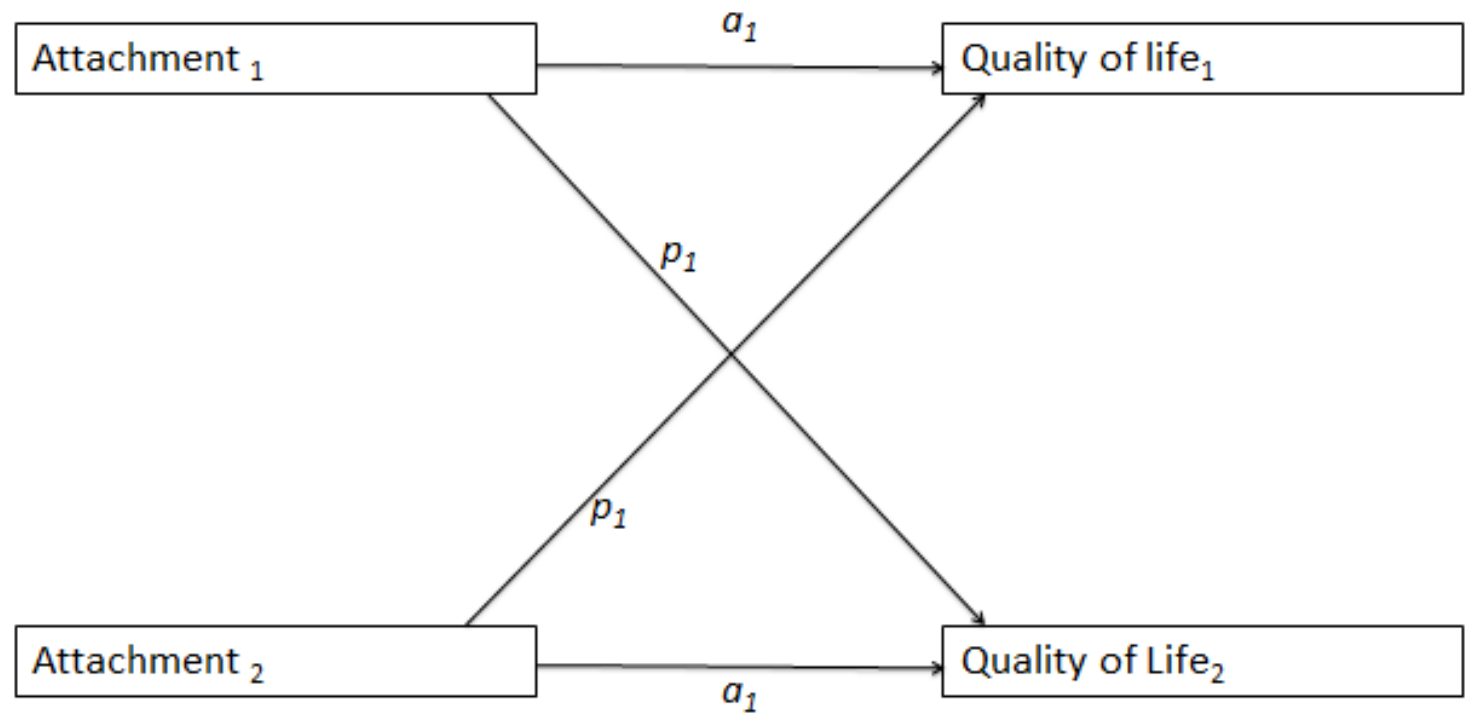

Figure 2. Example APIM interaction model.

Note. ${ }_{1}$ and ${ }_{2}$ refer to the different members of the dyad (person 1 and person 2).

$a=$ actor, $p=$ partner 
patients are referred to as spouses, and the term partner is reserved for referring to the partner effect as defined above. APIM simultaneously estimates actor and partner effects on an outcome variable. Analyses were completed using the Statistical Package for the Social Science (SPSS) version 22 .

Within APIM, dyads can be distinguishable or indistinguishable. Dyads are considered distinguishable when there is a consistent within-dyad variable: a variable that reliably differs between dyad members, but this variance is the same across the sample of dyads. For example, in the present study, dyads can be distinguished by role (patient versus spouse), because each dyad contains both a patient and a spouse. For distinguishable dyads, there are four paths that are estimated in the model, this is referred to as the two-intercept model, as displayed in Figure 3: the actor effect for patients, the actor effect for spouses, the partner effect for patients, and the partner effect for spouses. The actor and partner paths are always clarified by referring to the outcome variable. For example, the spouse partner effect $\left(p_{\text {spouse }}\right)$ refers to the effect of the patient predictor on the spouse's outcome variable.

Interaction model. APIM analyses with distinguishable dyads begin with the interaction model, see Figure 2 (Kenny et al., 2006). The interaction model estimates actor and partner effects averaged across dyad members, as well as whether the actor and partner effects are significantly moderated by the distinguishing variable, such as role (Kenny et al., 2006). The actor and partner predictor variables and the distinguishing variable(s) are each entered as main effects, as well as the interaction between the distinguishing variable with each of the actor and partner predictors. The results of this analysis indicates whether there are overall actor and partner effects of a predictor on an outcome, regardless of the distinguishing variable (i.e., role). Additionally, if there is a significant interaction between the distinguishing variable and the actor 


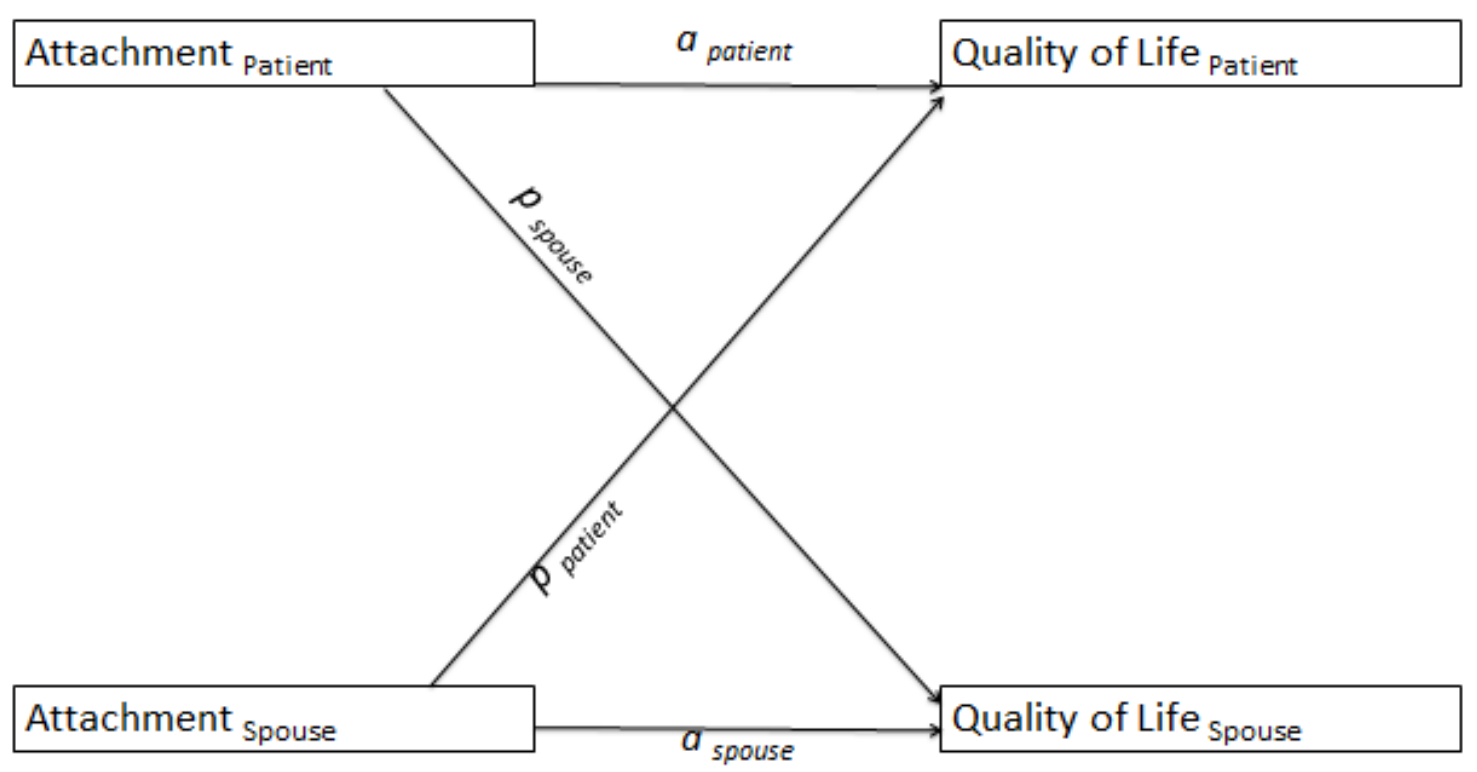

Figure 3. Example APIM two-intercept model.

Note. $a=$ actor, $p=$ partner 
and/or partner effect, this indicates that the magnitude of the estimated main effect is significantly different according to the distinguishing variable (i.e., role). A significant interaction term necessitates the use of the two-intercept model, as shown in Figure 3.

Two-intercept model. The two-intercept model is an extension of the interaction model, however, it allows for heterogeneity of variance across levels of the distinguishing variable (Kenny et al., 2006). That is, for the present study, the two-intercept model allows for different variances between patients and spouses. In this model, the distinguishing variable (i.e., role) is coded as a repeated measures variable, which allows for the significance of the actor and partner effects to be different for each dyad member. The results of this analysis indicate the significance of each of the actor and partner effects for each of the patients and spouses (specifically, the patient actor, spouse actor, patient partner, and spouse partner effects). If there is no significant interaction in the interaction model, the two-intercept model can still be examined, however, any differences between actor or partner effects by the distinguishing variable cannot be meaningfully interpreted as they are not statistically different. Therefore, for the purpose of the present study, the two-intercept model was only examined if the interaction model indicated a statistical difference in the actor or partner effects between patients and spouses.

Mediation. The standard APIM can be extended to include a mediator variable; this model has been referred to as the actor partner interdependence mediation model or APIMeM (Ledermann, Macho, \& Kenny, 2011). Tests of mediation in APIMeM include testing the paths from the predictor variable to the mediator (path $a$ ), the mediator to the outcome variable (path $b$ ), the predictor variable to the outcome variable (path $c$ ), and the same path after taking into consideration the mediator (path $c^{\prime}$ ). Mediation occurs under circumstances in which path $a b$ is significant and the $c$ ' path is significantly smaller than the $c$ path (Ledermann et al., 2011). The 
APIM framework is used to assess each path and a Sobel test is used to determine whether mediation has occurred (Kenny et al., 2006). Within APIMeM, actor and partner effects can be mediated with each of actor or partner mediators. For the interaction model, there are two effects to be mediated and two possible mediators, resulting in four possible paths of mediation; this is depicted in Figure 4. Specifically, there may be an actor-actor indirect effect in which the actor effect is mediated by the actor mediator; there may be a partner-partner effect in which the actor effect is mediated by the partner mediator; there may be a partner-actor effect in which the partner effect is mediated by the actor mediator; and there may be an actor-partner effect in which the partner effect is mediated by the partner mediator.

For the two-intercept model, there are four effects to be mediated and two possible mediators, resulting in eight possible paths of mediation. This is depicted in Figure 5. The eight paths are the same as above, for the interaction model, but specified for member of the dyad (for example, patient actor-actor effect and spouse actor-actor effect). Each of these paths of mediation are tested with the Sobel test.

Potential covariates. Relevant demographic and medical variables were included as covariates in the aforementioned analyses. While some of the extant literature has found positive associations between age and health-related quality of life (Sammarco, 2009; Song et al., 2011; von Gruenigen et al., 2010), a study on cervical cancer patients failed to demonstrate an association between age or stage of cancer and quality of life (Pasek, Suchocka, \& Urbański, 2013). A positive association between income and quality of life among both cancer patients and their spouses has been demonstrated (Mosher et al., 2013; Song et al., 2011). Education has been inconsistently associated with quality of life among couples facing cancer (Mosher et al., 2013; Song et al., 2011; Vacek, Winstead-Fry, Secker-Walker, Hooper, \& Plante, 2003). Moreover, 


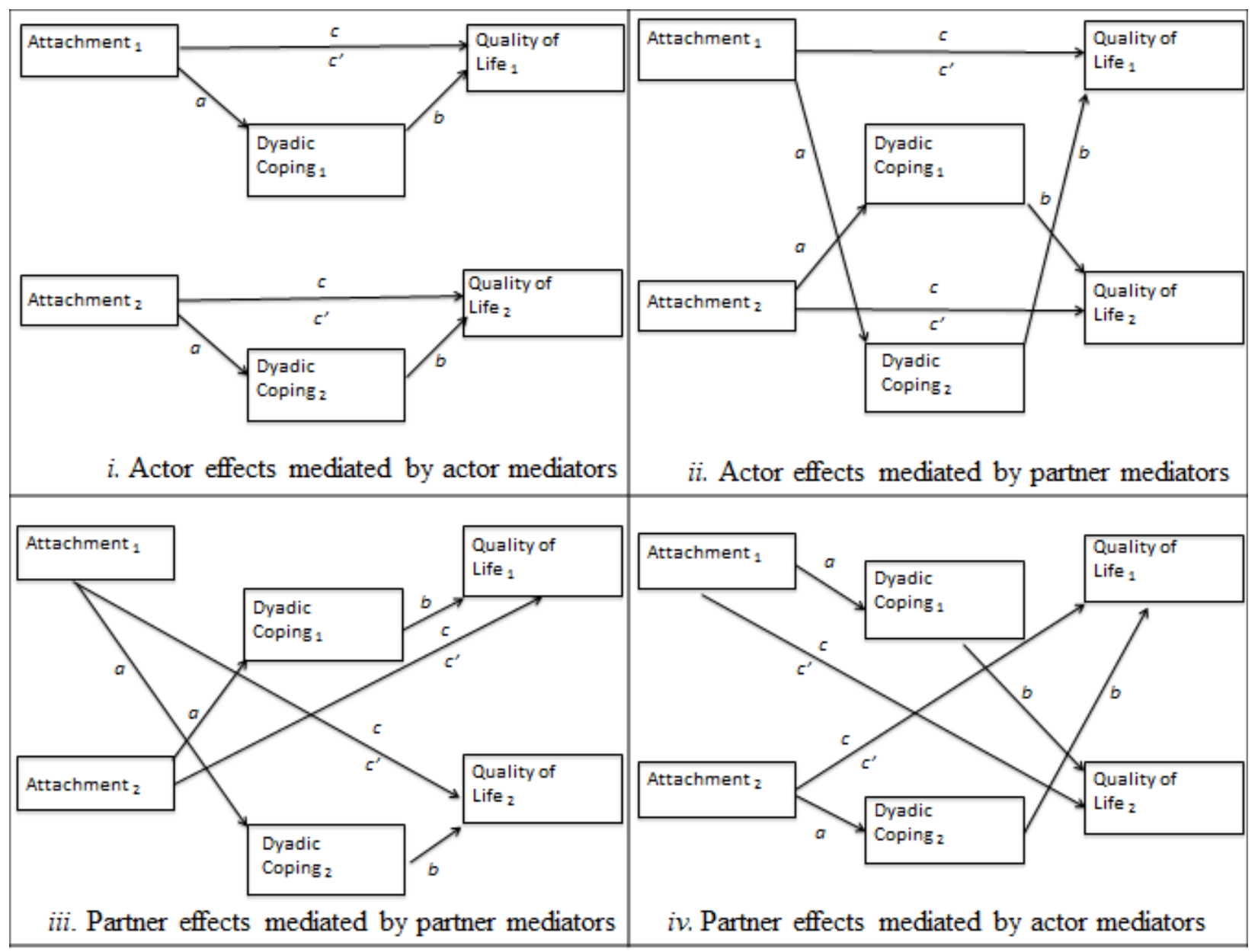

Figure 4. Example APIMeM interaction model.

Note. Each model will be run averaged across members of the dyad, prodvcing four models.

1 and 2 refer to different members of the dyad; a, b, c, and c' refer to the different paths within mediation 


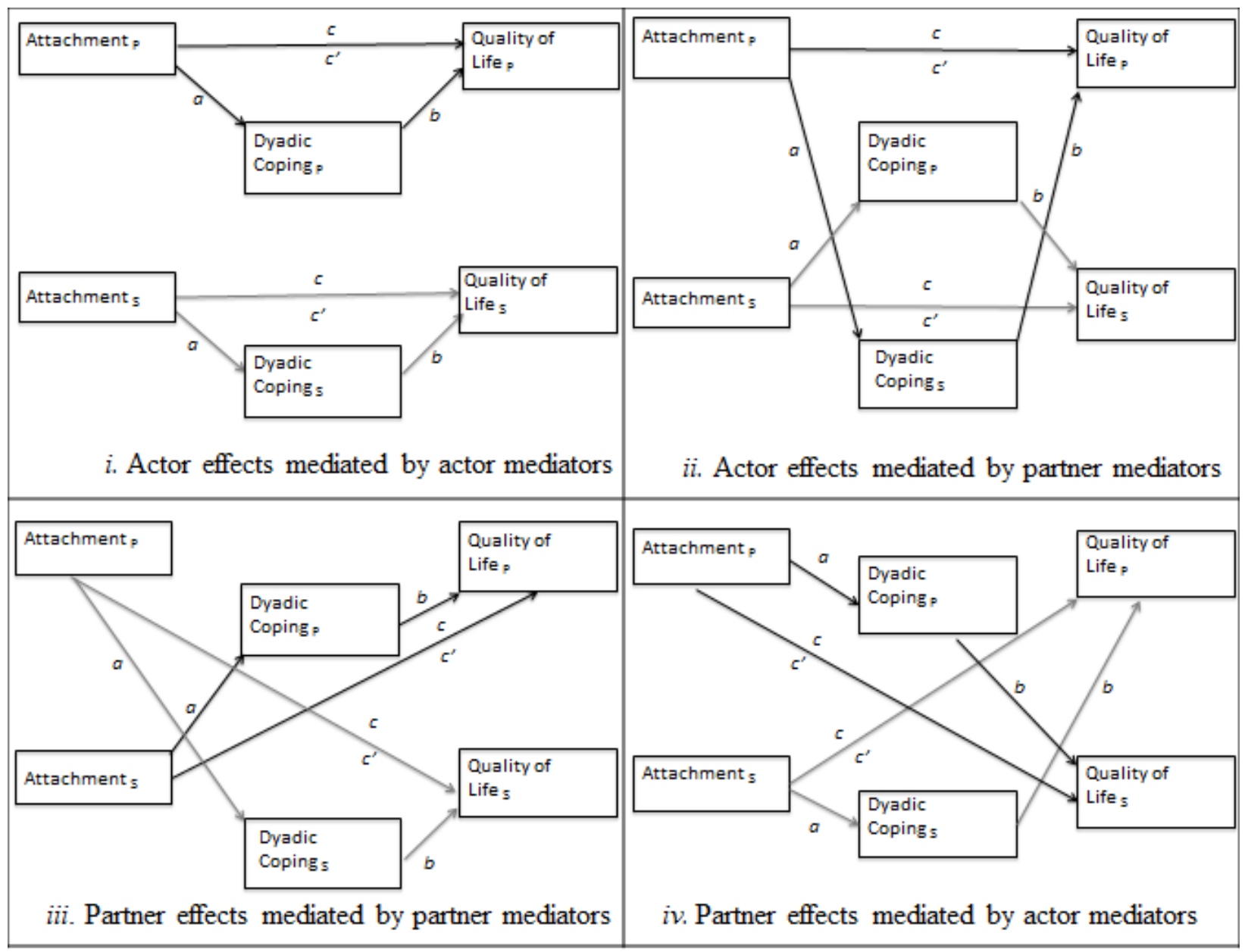

Figure 5. Example APIMeM interaction model.

Note. Each model will be run separately for patients and spouses, producing eight models.

$\mathrm{P}=$ patient and $\mathrm{g}$ = spouse; $\mathrm{a}, \mathrm{b}, \mathrm{c}$, and $\mathrm{c}$ ' refer to the different paths within mediation 
stage of illness, family history, and treatment modality have all been inconsistently associated with quality of life (Sammarco, 2009; Vacek et al., 2003). The associations of aforementioned demographic and medical variables with outcome variables were examined, and the main analyses included those covariates found to be significantly related.

Aim 1. It was expected that there would be significant actor (Hypothesis 1) and partner (Hypothesis 2) effects of attachment on patients' and spouses' quality of life, such that, greater anxious and avoidant attachment were expected to be associated with worse quality of life. The moderating influence of role was explored (Exploratory Analysis 1).

Associated analyses. Eight separate analyses examined the effects of anxious attachment and avoidant attachment on physical, social, emotional, and functional well-being. The APIM was used to estimate actor and partner effects. Each set of analyses began with the interaction model to determine the significance of actor, partner, and interaction effects. The fixed effects included relevant covariates, role, actor attachment, partner attachment, and the interactions between each of actor and partner attachment and role. Results of these interaction analyses revealed whether there were significant overall actor or partner effects as well as whether there was significant moderation by role on quality of life. As the results did not reveal any significant interaction between actor or partner attachment and role, two-intercept analyses were not necessary.

Aim 2. It was expected that there would be significant actor (Hypothesis 3) and partner (Hypothesis 4) effects of positive and negative dyadic coping on patients' and spouses' quality of life. The moderating influence of role was explored (Exploratory Analysis 2).

Associated analyses. Eight separate analyses examined the effects of positive and negative dyadic coping on physical, social, emotional, and functional well-being. The APIM was 
used to estimate actor and partner effects. As with the above analyses, each set of analyses began with the interaction model to determine the significance of actor, partner, and interaction effects. The fixed effects included relevant covariates, role, actor positive or negative dyadic coping, partner positive or negative dyadic coping, and the interactions between dyadic coping and role. Results of these interaction analyses revealed whether there were significant overall actor or partner effects as well as whether there was significant moderation by role for the effects of dyadic coping on quality of life. As the results did not reveal any significant interaction between actor or partner dyadic coping and role, two-intercept analyses were not necessary.

Aim 3. It was expected that the actor and partner effects of greater insecure attachment on more impaired quality of life (Hypothesis 6) would be significantly mediated by positive and negative dyadic coping. The moderating influence of role was explored (Exploratory Analysis 3).

Associated analyses. Although bootstrapping is currently the leading method of testing for mediation in non-dyadic analyses, within APIMeM, Sobel tests were used to test for mediation in the present study. Bootstrapping has been used for APIMeM before; however, it can only be accomplished through structural equation modeling, which would demand a larger sample size than was feasible for the current study (Ledermann et al., 2011). Furthermore, bootstrapping for APIMeM in SPSS is not currently possible. Consistent with the published literature on APIMeM (i.e. Manne \& Badr, 2010) and based on personal communications with David A. Kenny, it was decided that Sobel testing would be used to assess for mediation in the present study for APIMeM. For Hypotheses 8 and 9, APIMeM was used to assess whether dyadic coping mediates the associations between attachment and quality of life.

As there were no significant interactions with role, the interaction model was used for all the APIMeM analyses. Sobel tests were used to examine whether significant mediation had 
occurred. Anxious and avoidant attachment as predictors were each assessed in separate analyses; physical, social, emotional, and functional well-being as outcomes were also each assessed in separate analyses; finally, positive and negative dyadic coping as mediators were each assessed in separate analyses. In total, 16 mediation models were tested. Given the number of analyses completed for each aim, to control for Type 1 error a conservative $p$ value of .01 was employed throughout.

\section{Power Analysis}

A power analysis was completed to determine the number of participants necessary to sufficiently power the present study. As elaborated below, the present study used dyadic data analyses. Consequently, the power analysis was conducted using an online program designed to estimate power for the Actor-Partner Interdependence Model analyses (Ackerman, Ledermann, \& Kenny, Manuscript in progress). An estimation of effect sizes was used from a study of the dyadic effects of emotion-focused coping on the quality of life of prostate cancer patients and their spouses (Lafaye et al., 2014). A number of analyses were completed by Lafaye and colleagues (2014) examining the effects of emotion-focused coping on quality of life across three time points; to be conservative, the smallest effect sizes for each of actor and partner effects from that publication were selected for the present power analysis. The estimated effect size for the actor effect was .25 , the estimated effect size for the partner effect was .19, the estimated correlation of actor and partner variables was .30, and the estimated correlation of the errors was also set at .30 . The results of the power analysis suggested that to achieve the desired power of .80 with an alpha of $.05,99$ dyads were needed. 


\section{Results}

\section{Recruitment}

Recruitment was completed at Princess Margaret Hospital in the Gynaecology Oncology Clinic. See Figure 6 for an overview of the study recruitment flow. Study staff approached 576 patients. Twenty-five were classified as ineligible because they were diagnosed with a borderline or non-invasive tumour, 42 were unable to speak or read English, a member of the healthcare team recommended against participation for 7 patients, and 171 did not have a partner. Of the 331 eligible patients, 116 declined, 46 indicated that their partner would not be interested, and 8 did not give permission to contact their partner. There were 161 coupled patients who expressed an interest in the study. Five did not provide consent (2 patients declined and study staff were unable to follow-up with 3 patients) and 9 had partners who declined to consent. There were 147 consented couples. This reflects a consent rate of $44.4 \%$. Twenty-six patients did not complete the study ( 2 patients passed away and 24 withdrew), which left a total of 121 patients who completed the questionnaire. Fifteen were excluded from the present couples-level analyses as their partner did not complete the questionnaire. A total sample of 106 patients and their spouses were included in the present study. This reflects a couples' response rate (the percent of eligible couples who completed, excluding the two deceased patients) of $32.2 \%$, and a couples' completion rate (the percent of consented couples who completed, excluding the two deceased patients) of $83.4 \%$. Recruitment took place over a period of 10 months.

\section{Descriptive Statistics and Preliminary Analyses}

One hundred and six couples were included in the present study $(N=212)$. Table 1 displays the demographics of the present sample, separated by patients and spouses. The average age was 59 years for patients and 61 years for spouses. Average duration of relationship was 29 


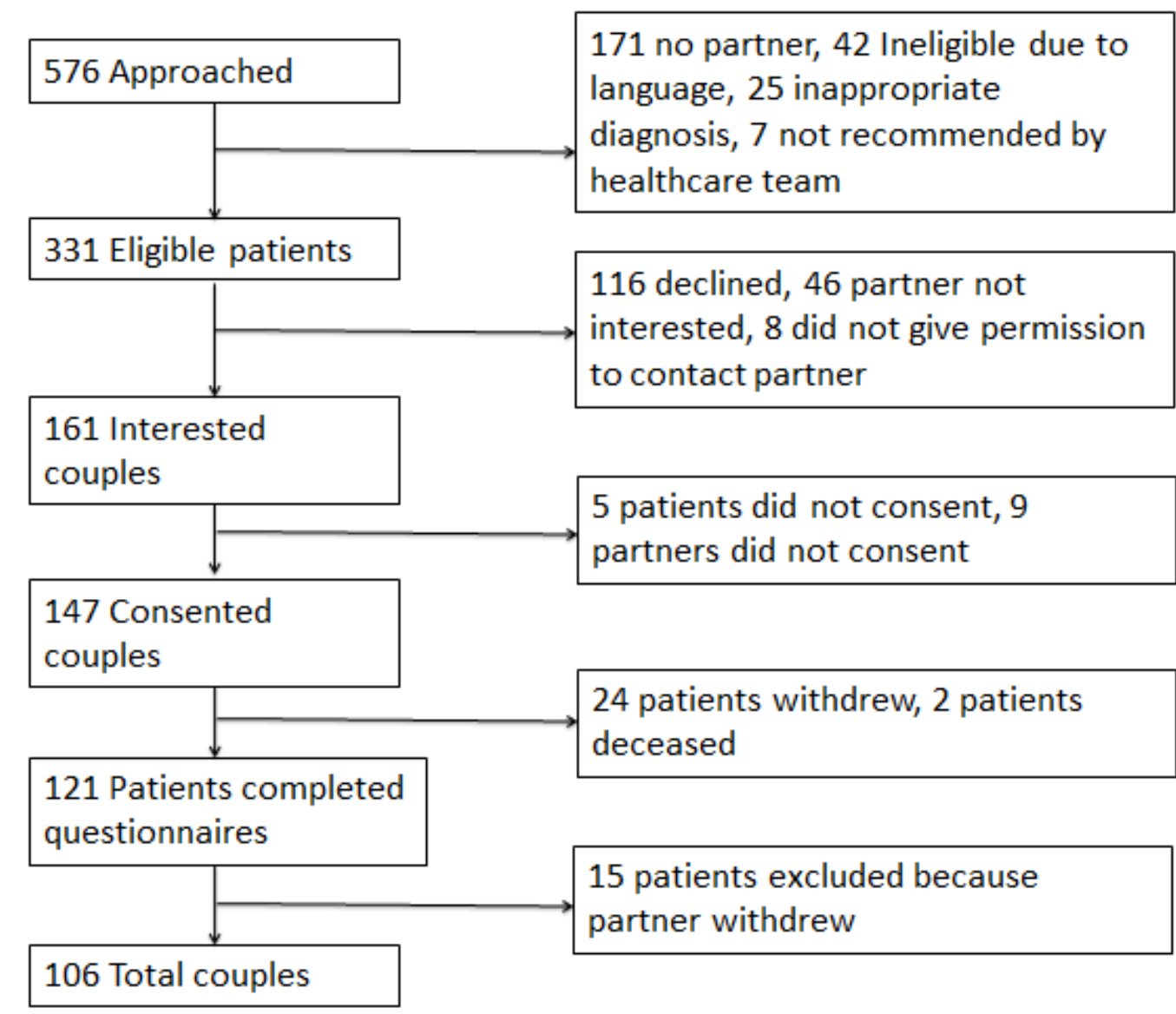

Figure 6. Participant recruitment. 
Table 1

Sample Demographics

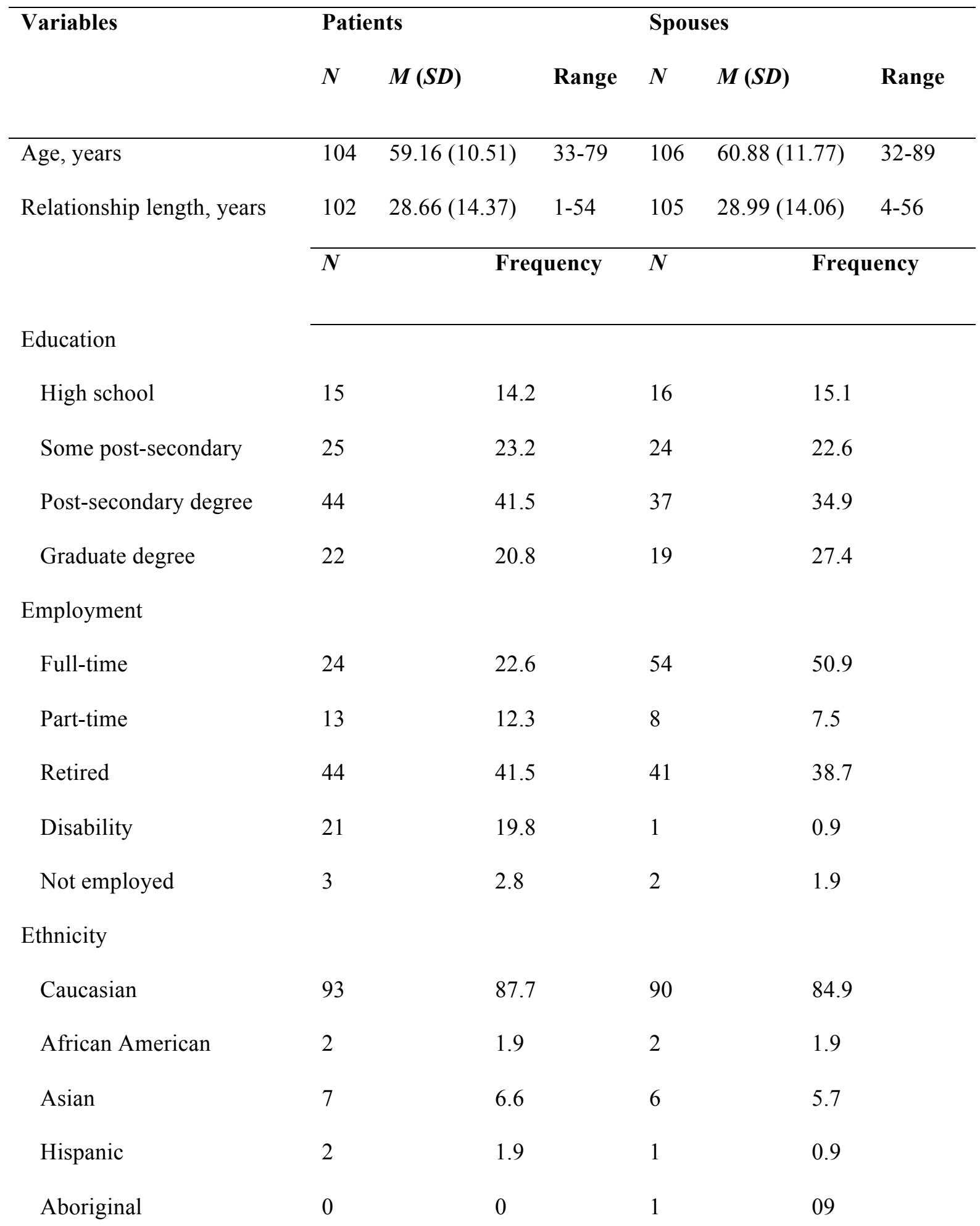


Other

Income

$0-40,000$

$40,000-75,000$

$75,000+$
22

2

42

36
1.9

39.6

20.8

34.0

Note. $M=$ mean, $S D=$ standard deviation

5

4.7 
years, with relationship length ranging from 1 to 56 years. There were 5 same-sex couples. The majority of the sample was Caucasian (85-88\%) and highly educated, with $62.3 \%$ of both patients and spouses reporting a college or university degree or higher education levels. There were no significant differences between patients and spouses on age, relationship length, education, or ethnicity. A chi-square analysis revealed significant differences between patients and spouses for employment, $\chi(4)=31.21, p=.001$. For patients, $35 \%$ were working full- or part-time, $42 \%$ were retired, and $20 \%$ were on disability. For spouses, $58 \%$ were working full- or part-time, $39 \%$ were retired, and $1 \%$ were on disability. A chi-square analysis also revealed significant differences between patients and spouses for income, $\chi(2)=16.10, p=.001$. Forty percent of patients reported an average income of between 0 to 40,000 dollars, whereas $45 \%$ of spouses reported their average annual income to be greater than 75,000 dollars.

Medical characteristics of the patients are displayed in Table 2. Majority of patients were diagnosed with late-stage illness- $71 \%$ of the sample were diagnosed with Stage 3 or 4 ovarian cancer. It had been an average of three and a half years since diagnosis. Ten percent of patients were receiving their first treatment for ovarian cancer, $4 \%$ were within 6 months of their first treatment, $9 \%$ were within 6 to 12 months of their first ovarian cancer treatment, $34 \%$ had completed their first ovarian cancer treatment more than one year prior, and $38 \%$ of participants had experienced a recurrence. Of the four participants who indicated 'other' for their current point in the cancer journey, one was palliative, one was scheduled for surgery to investigate a possible recurrence, and two did not specify. Fifty seven percent of patients were not actively receiving treatment, $19 \%$ were undergoing chemotherapy, and $15 \%$ were undergoing both surgery and chemotherapy. Of the participants not currently receiving treatment, $36 \%$ were more than a year away from their initial treatment and 32\% had experienced recurrence. Additionally, 
Table 2

Medical characteristics

\begin{tabular}{lll}
\hline Variables & $N$ & $\%$
\end{tabular}

Stage of cancer

Stage 1

18

17.0

Stage 2

5

4.7

Stage 3

57

53.8

Stage 4

18

17.0

Treatment progress

Primary treatment of ovarian cancer

11

10.4

Within 6 months of completing first treatment

4

3.8

Within 6-12 months of completing first treatment

10

9.4

Greater than one year from completed first treatment

36

34.0

After recurrence of cancer

40

37.7

Other

4

3.8

Current treatment

Surgery only

7

6.6

Chemotherapy only

20

18.9

Surgery and chemotherapy

16

15.1

Surgery, radiation, and chemotherapy

2

1.9

Not currently receiving treatment

60

56.6

Past treatment

Surgery only

10

9.4

Chemotherapy only

3

2.8

Surgery and chemotherapy

62

58.5 
Surgery and radiation therapy

Surgery, radiation, and chemotherapy

Not applicable

BRCA

Positive

Negative

Note. ${ }^{\mathrm{a}}$ These percentages do not add up to 100 because the remainder of participants either had not undergone genetic testing or did not report their test results.

$M=$ mean, $S D=$ standard deviation
3

13

12.3

13

12.3

17

16

46

$43.4^{\mathrm{a}}$ 
59 out of the 60 patients not current receiving treatment had received treatment in the past: $73 \%$ had surgery and chemotherapy, $13 \%$ had surgery, radiation, and chemotherapy, $5 \%$ had surgery only, and 3\% had each of chemotherapy only or surgery and radiation therapy. Amongst all the patients, the majority reported having been treated with surgery and chemotherapy in the past.

To determine covariates to be included in the statistical models, the correlations and comparisons of means between quality of life (physical, social, emotional, and functional wellbeing) and demographic and medical variables were examined. Age was significantly correlated with social well-being, $r=.21, p=.002$, and functional well-being, $r=.15, p=.035$, such that older age was associated with better social and functional well-being. Relationship duration was significantly correlated with social well-being, $r=.21, p=.002$, such that longer relationship duration was associated with better social well-being. Employment was recoded into working (full-time or part-time) and not working (retired, disability, or unemployed). An independent samples $t$-test revealed significant effects of employment on physical well-being, $t(204.42)=$ $3.16, p=.002, M D_{\text {working }- \text { not working }}=2.15$, social well-being, $t(208)=-2.62, p=.009, M D_{\text {working }}$ not working $=-1.99$, emotional well-being, $t(205)=2.65, p=.009, M D_{\text {working }- \text { not working }}=1.79$, and functional well-being, $t(208)=2.06, p=.04, M D_{\text {working }- \text { not working }}=1$.64. Individuals who were employed reported significantly better physical, emotional, and functional well-being. Individuals who were not currently working reported significantly better social well-being. Oneway analyses of variance found non-significant effects of education, ethnicity, and income on all quality of life subscales.

Current treatment was regrouped into active treatment or no current treatment. An independent samples $t$-test revealed significant effects of current treatment on physical wellbeing, $t(75.24)=-3.15, p=.002$. Individuals not currently receiving treatment reported 
significantly better physical well-being $(M=22.98, S D=4.94)$ than individuals currently receiving active treatment $(M=19.18, S D=6.77)$. Treatment progress was regrouped into primary treatment of ovarian cancer, disease free interval, and after cancer recurrence. A oneway analysis of variance revealed a significant effect of treatment progress on physical wellbeing, $F(2,96)=4.06, p=.02$. Individuals within their primary treatment for ovarian cancer reported significantly worse physical well-being $(M=16.82, S D=6.78)$ than individuals within a disease-free interval $(M=22.33, S D=5.52)$. One-way analyses of variance found nonsignificant effects of cancer stage and past treatment (which was regrouped into single mode of treatment, multimodal treatment, or no past treatment) on all quality of life subscales. Nonsignificant correlations were found for time since diagnosis and all quality of life subscales. BRCA test result was not examined as a covariate as BRCA test results were only reported by $60 \%$ of the sample. In sum, physical well-being was associated with being employed, not being on current treatment, and less treatment progress; social well-being was associated with older age, longer relationship duration, and being employed; emotional well-being was associated with being employed; and functional well-being was associated with older age and being employed.

Table 3 displays the means and standard deviations of attachment, dyadic coping, and quality of life variables for patients and spouses. The quality of life scores can be compared to existing data to further understand and contextualize the present results; a previous investigation into minimally important differences for the FACT scales determined that a two-point difference on one of the FACT subscales can be considered a meaningful difference (Yost \& Eton, 2005). Compared to the sample of ovarian cancer patients in the validation study of the FACT-O, patients in the present sample reported comparable physical $(M=22.25$ versus $M=21.39)$, social ( $M=23.33$ versus $M=22.52)$, and emotional well-being $(M=18.52$ versus $M=16.96$; 
Table 3

Descriptive Statistics of Primary Study Variables

\begin{tabular}{llc}
\hline Variables & Patients & Mean (SD) \\
& & Spouses \\
\hline Anxious attachment & $2.13(1.18)$ & $2.15(0.89)$ \\
Avoidant attachment & $2.48(1.17)$ & $2.63(1.14)$ \\
Positive dyadic coping & $3.90(0.59)$ & $3.78(0.53)$ \\
Negative dyadic coping & $2.03(0.50)$ & $1.99(0.47)$ \\
Physical well-being & $21.39(6.05)^{*}$ & $25.56(2.59)^{*}$ \\
Social well-being & $22.51(4.71)^{*}$ & $18.93(5.81)^{*}$ \\
Emotional well-being & $16.96(5.06)^{*}$ & $20.62(4.01)^{*}$ \\
Functional well-being & $17.93(5.92)^{*}$ & $20.72(5.35)^{*}$ \\
\hline
\end{tabular}

Note. $S D=$ standard deviation

* Significant difference between patients and spouses, $p<.001$ 
Basen-Engquist et al., 2001). Patients in the present sample endorsed less functional well-being ( $M=20.94$ versus $M=17.93$; Basen-Engquist et al., 2001). Compared to a sample of the general United States adult population, spouses in the present sample reported comparable emotional ( $M$ $=20.62$ versus $M=19.9)$ and social well-being $(M=19.1$ versus $M=18.93$; Brucker et al., 2005). Spouses in the present sample endorsed better physical $(M=25.56$ versus $M=22.7)$ and functional well-being ( $M=20.72$ versus $M=18.5$; Brucker et al., 2005) compared to normative data.

Paired samples $t$-tests revealed significant differences between patients and spouses for physical well-being, $t(103)=-6.36, p<.001$, social well-being, $t(104)=5.61, p<.001$, emotional well-being, $t(101)=-5.84, p<.001$, and functional well-being, $t(104)=-4.20, p<$ .001. Patients reported worse physical, emotional, and functional well-being. Spouses reported worse social well-being (see Table 3 for means). There were no significant differences between patients and spouses for attachment or dyadic coping. Prior to conducting APIM analyses, attachment and dyadic coping, as predictor variables, were centered to allow for a more straightforward interpretation of the results (Kenny et al., 2006).

Table 4 displays the correlations between attachment, dyadic coping, and quality of life for patients and spouses. Anxious attachment was positively correlated with avoidant attachment and negative dyadic coping, and negatively correlated with positive dyadic coping and physical, social, emotional, and functional well-being $(p \mathrm{~s}<.001)$. Avoidant attachment was positively correlated with negative dyadic coping, and negatively correlated with positive dyadic coping and social, emotional, and functional well-being $(p s<.001)$. Avoidant attachment was not correlated with physical well-being. Positive dyadic coping was negatively correlated with negative dyadic coping, and positively correlated with social and functional well-being ( $p$ s $<.01$ 
Table 4

Correlations Between Study Variables

\begin{tabular}{|c|c|c|c|c|c|c|c|c|}
\hline & 1 & 2 & 3 & 4 & 5 & 6 & 7 & 8 \\
\hline 1. Anxious attachment & .14 & $.55 * * *$ & $-.33 * * *$ & $.34 * * *$ & $-.18 * *$ & $-.41 * * *$ & $-.27 * * *$ & $-.32 * * *$ \\
\hline 2. Avoidant attachment & & $.33 * * *$ & $-.54 * * *$ & $.29 * * *$ & -.14 & $-.46 * * *$ & $-.25 * * *$ & $-.36 * * *$ \\
\hline 3. Positive dyadic coping & & & $.35 * * *$ & $-.43 * * *$ & .02 & $.53 * * *$ & .08 & $.21 * *$ \\
\hline 4. Negative dyadic coping & & & & $.52 * * *$ & $-.14^{*}$ & $-.26 * * *$ & -.13 & $-.20 * * *$ \\
\hline 5. Physical well-being & & & & & -.17 & .06 & $.49 * * *$ & $.60 * * *$ \\
\hline 6. Social well-being & & & & & & .09 & $.21 * *$ & $.38 * * *$ \\
\hline 7. Emotional well-being & & & & & & & -.09 & $.62 * * *$ \\
\hline 8. Functional well-being & & & & & & & & $.21 *$ \\
\hline
\end{tabular}

Note. Intraclass correlations between dyad members are displayed on the diagonal.

$* p<.05, * * p<.01, * * * p<.001$ 
to .001). Positive dyadic coping was not associated with physical or emotional well-being. Negative dyadic coping was negatively associated with physical, social, and functional wellbeing ( $p$ s $<.05$ to .001 ). Negative dyadic coping was not significantly associated with emotional well-being. Intraclass correlations between dyad members are displayed on the diagonal in Table 4. There were significant associations between dyad members for avoidant attachment, positive dyadic coping, negative dyadic coping, and functional quality of life. These results indicate that the more one individual endorsed greater avoidant attachment, positive and negative dyadic coping, and functional well-being, the more their partner also reported greater avoidant attachment, positive and negative dyadic coping, and functional well-being There were nonsignificant associations between dyad members for anxious attachment, as well as physical, social, and emotional well-being. These results suggest that the extent to which one individual reports anxious attachment or physical, social, and emotional well-being is not associated with their partners' anxious attachment or physical, social, and emotional well-being.

\section{Aim 1: To Examine the Association between Attachment and Quality of Life}

It was hypothesized that greater actor avoidant and anxious attachment would be associated with lower actor health-related quality of life. It was further hypothesized that there would be a significant negative relationship between greater actor avoidant and anxious attachment and less partner health-related quality of life. Avoidant and anxious attachment were examined in separate analyses for each of physical, social, emotional, and functional well-being. The APIM results for avoidant attachment and anxious attachment are displayed in Table 5 and 6 respectively.

Physical well-being. Employment, current treatment, and treatment progress were included as covariates in analyses of physical well-being. For avoidant attachment, an interaction 
Table 5 .

APIM Interaction Model Estimating Effects of Avoidant Attachment on Quality of Life

\begin{tabular}{|c|c|c|c|c|c|c|c|c|}
\hline \multirow[b]{2}{*}{ Predictors } & \multicolumn{2}{|c|}{ PWB } & \multicolumn{2}{|c|}{ SWB } & \multicolumn{2}{|c|}{ EWB } & \multicolumn{2}{|c|}{ FWB } \\
\hline & $b$ & $S E$ & $b$ & $S E$ & $\boldsymbol{b}$ & $S E$ & $b$ & $S E$ \\
\hline Role & $1.90 * * *$ & .35 & $-1.83 * * *$ & .33 & $1.72 * * *$ & .31 & $.86^{* *}$ & .32 \\
\hline Age & - & - & $.11^{* *}$ & .04 & - & - & $.15^{* * *}$ & .04 \\
\hline Relationship duration & - & - & .04 & .03 & - & - & - & - \\
\hline Employment & $-.54 *$ & .24 & $-.63 *$ & .31 & $-.64 *$ & .28 & $-1.71 * * *$ & .34 \\
\hline Current treatment & .53 & .47 & - & - & - & - & - & - \\
\hline Treatment progress & .48 & .35 & - & - & - & - & - & - \\
\hline Actor avoidant attachment & -.56 & .30 & $-2.11 * * *$ & .29 & $-1.23 * * *$ & .28 & $-1.91 * * *$ & .31 \\
\hline Partner avoidant attachment & -.07 & .30 & -.03 & .29 & .16 & .28 & .08 & .31 \\
\hline Role ${ }^{*}$ Actor avoidant attachment & .03 & .30 & -.17 & .30 & -.13 & .29 & -.15 & .35 \\
\hline Role * Partner avoidant attachment & .06 & .30 & -.17 & .30 & -.16 & .29 & .19 & .35 \\
\hline
\end{tabular}

${ }^{*} p<.05, * * p<.01, * * * p<.001$ 
Table 6.

APIM Interaction Model Estimating Effects of Anxious Attachment on Quality of Life

\begin{tabular}{|c|c|c|c|c|c|c|c|c|}
\hline & \multicolumn{2}{|c|}{ PWB } & \multicolumn{2}{|c|}{ SWB } & \multicolumn{2}{|c|}{ EWB } & \multicolumn{2}{|c|}{ FWB } \\
\hline Predictors & b & $S E$ & $b$ & $S E$ & $b$ & $S E$ & b & $S E$ \\
\hline Role & $1.89 * * *$ & .35 & $-2.02 * * *$ & .34 & $1.61 * * *$ & .31 & $.68^{*}$ & .32 \\
\hline Age & - & - & .08 & .04 & - & - & $.13 * *$ & .04 \\
\hline Relationship duration & - & - & .04 & .03 & - & - & - & - \\
\hline Employment & $-.51^{*}$ & .23 & $-.64 *$ & .31 & $-.64^{*}$ & .28 & $-1.71 * * *$ & .35 \\
\hline Current treatment & .77 & .45 & - & - & - & - & - & - \\
\hline Treatment progress & .33 & .35 & - & - & - & - & - & - \\
\hline Actor anxious attachment & $-.80 * *$ & .29 & $-2.01 * * *$ & .34 & $-1.33 * * *$ & .30 & $-1.68 * * *$ & .36 \\
\hline Partner anxious attachment & -.35 & .36 & -.48 & .32 & .24 & .32 & -.37 & .36 \\
\hline Role*Actor anxious attachment & .03 & .29 & -.24 & .35 & -.07 & .31 & .08 & .38 \\
\hline Role*Partner anxious attachment & .45 & .36 & .06 & .32 & -.20 & .32 & .22 & .37 \\
\hline
\end{tabular}


model was run to determine the significance of the actor and partner effects, and whether these effects were moderating by role. The main effects of role, employment, current treatment, treatment progress, actor avoidant attachment, and partner avoidant attachment, as well as the interaction terms of role by actor avoidant attachment and role by partner avoidant attachment were entered as fixed effects.

Results demonstrated a significant effect for role, $b=1.90, p<.001$, and employment, $b$ $=-0.54, p<.05$. Applying the conservative $p$-value of .01 to correct for multiple analyses, only role remained significant. These results demonstrated that being a patient was associated with poorer physical well-being. The actor and partner effects were not significant, suggesting the association between an individual's physical well-being was not significantly associated with one's own or their partner's avoidant attachment. Additionally, the interaction effects were nonsignificant, suggesting the magnitude of the actor and partner effects of avoidant attachment were not significantly different for patients and spouses. As there were no significant interactions, a two-intercept model was not completed. The pseudo $R^{2}$ for this model was .215; that is, the effects of role, employment, current treatment, treatment progress, actor avoidant attachment, and partner avoidant attachment accounted for $21.5 \%$ of the variance in physical well-being.

For anxious attachment, an interaction model was run to determine the significance of the actor and partner effects, and whether these effects were moderating by role. The main effects of role, employment, current treatment, treatment progress, actor anxious attachment, and partner anxious attachment, as well as the interaction terms of role by actor anxious attachment and role by partner anxious attachment were entered as fixed effects. Results demonstrated a significant effect for role, $b=1.85, p<.001$, and employment, $b=-.51, p<.05$. Additionally, there was a 
significant actor effect of anxious attachment, $b=-0.80, p<.01$. Correcting for multiple analyses, role and actor anxious attachment remained significant. These results suggest that being a patient was associated with poorer physical well-being. The significant actor effect indicates that an individual's own greater anxious attachment was associated with one's own less physical well-being. The partner effect was not significant suggesting the association between an individual's anxious attachment was not significantly associated with their partner's physical well-being. Additionally, the interaction effects were non-significant, suggesting the magnitude of the actor and partner effects for anxious attachment were not significantly different for patients and spouses. As there were no significant interactions, a two-intercept model was not completed. The pseudo $R^{2}$ for this model was .242; that is, the effects of role, employment, current treatment, treatment progress, actor anxious attachment, and partner anxious attachment accounted for $24.2 \%$ of the variance in physical well-being.

Social well-being. Age, relationship duration, and employment were entered as covariates for analyses of social well-being. The interaction model for avoidant attachment revealed significant main effects for role, $b=-1.83, p<.001$, age, $b=0.11, p<.01$, employment, $b=-0.63, p<.05$, and actor avoidant attachment, $b=-2.11, p<.001$. Correcting for multiple analyses, role, age, and actor avoidant attachment remained significant. These results suggest that being a patient and older age were associated with better social well-being. Additionally, the actor effect suggests that an individual's own greater avoidant attachment was associated with one's own worse social well-being. The partner and the interaction effects were not significant. As there was no evidence that the magnitude of the actor and partner effects differed by role, a two-intercept model was not completed. The pseudo $R^{2}$ for this model was 0.346 ; that is, the 
effects of role, age, relationship duration, employment, actor avoidant attachment, and partner avoidant attachment accounted for $34.6 \%$ of the variance in social well-being.

The interaction model for anxious attachment revealed significant main effects for role, $b$ $=-2.02, p<.001$, employment, $b=-0.64, p<.05$ and actor anxious attachment, $b=-2.01, p<$ .001. Correcting for multiple analyses, role and actor anxious attachment remained significant. These results suggest that being a patient was associated with better social well-being. Additionally, the actor effect suggests that an individual's own greater anxious attachment was associated with one's own poorer social well-being. The partner and interaction effects were not significant. As there was no evidence that the actor or partner effects differed in magnitude by role, a two-intercept model was not completed. The pseudo $R^{2}$ for this model was 0.295 ; that is, the effects of role, age, relationship duration, family history, employment, actor anxious attachment, and partner anxious attachment accounted for $29.5 \%$ of the variance in social wellbeing.

Emotional well-being. Employment was entered as a covariate for analyses of emotional well-being. The interaction model for avoidant attachment revealed significant main effects of role, $b=1.72, p<.001$, employment, $b=-0.64, p<.05$, and actor avoidant attachment, $b=$ $1.23, p<.001$. Correcting for multiple analyses, role and actor avoidant attachment remained significant. These results suggest that being a patient was associated with worse emotional wellbeing. Additionally, the actor effect suggests that an individual's own greater avoidant attachment was associated with one's own poorer emotional well-being. The partner and interaction effects were not significant. As the interaction effects were not significant, a twointercept model was not completed. The pseudo $R^{2}$ for this model was 0.212 ; that is, the effects 
of role, employment, actor avoidant attachment, and partner avoidant attachment accounted for $21.2 \%$ of the variance in emotional well-being.

The interaction model for anxious attachment revealed significant main effects for role, $b$ $=1.61, p<.001$, employment, $b=-0.64, p<.05$, and actor anxious attachment, $b=-1.33, p<$ .001. Correcting for multiple analyses, role and actor anxious attachment remained significant. These results suggest that being a patient was associated with less emotional well-being. Additionally, the actor effect suggests that an individual's own greater anxious attachment was associated with one's own less emotional well-being. The partner and interaction effects were non-significant. As the interaction effects were not significant, a two-intercept model was not completed. The pseudo $R^{2}$ for this model was 0.208 ; that is, the effects of role, employment, actor anxious attachment, and partner anxious attachment accounted for $20.8 \%$ of the variance in emotional well-being.

Functional well-being. Age and employment were entered as covariates for analyses of functional well-being. The interaction model for avoidant attachment revealed significant main effects for role, $b=0.86, p<.01$, age, $b=0.15, p<.001$, employment, $b=-1.71, p<.001$ and actor avoidant attachment, $b=-1.91, p<.001$. Correcting for multiple analyses, each of these effects remained significant. These results suggest that being a patient, younger age, and not being employed were associated with less functional well-being. Additionally, the actor effect suggests that an individual's own greater avoidant attachment was associated with one's own less functional well-being. The partner and interaction effects were non-significant. As the interaction effects were not significant, a two-intercept model was not completed. The pseudo $R^{2}$ for this model was 0.257 ; that is, the effects of role, age, employment, actor avoidant attachment, and partner avoidant attachment accounted for $25.7 \%$ of the variance in functional well-being. 
The interaction model for anxious attachment revealed significant main effects for role, $b$ $=0.68, p<.05$, age, $b=0.13, p<.01$, employment, $b=-1.71, p<.001$, and actor anxious attachment, $b=-1.68, p<.001$. Correcting for multiple analyses, age, employment, and actor anxious attachment remained significant. These results suggest that younger age and not being employed were associated with less functional well-being. Additionally, the main effect suggests that an individual's own greater anxious attachment was associated with one's own less functional well-being. The partner and interaction effects were not significant. As the interaction effects were not significant, a two-intercept model was not completed. The pseudo $R^{2}$ for this model was 0.209; that is, the effects of role, age, employment, actor anxious attachment, and partner anxious attachment accounted for $20.9 \%$ of the variance in functional well-being.

Summary. In sum, there were significant actor effects of anxious attachment for physical, social, emotional, and functional well-being. There were also significant actor effects of avoidant attachment for social, emotional, and functional well-being. The actor effect of avoidant attachment for physical well-being was not significant. There was no evidence of significant partner effects nor was there evidence that the magnitude of the actor or partner effects differed for patients and spouses. These models accounted for between $21-35 \%$ of variance in well-being.

\section{Aim 2: To Examine the Association between Dyadic Coping and Quality of Life}

It was hypothesized that greater actor positive dyadic coping and less actor negative dyadic coping would be associated with greater actor health-related quality of life. It was further hypothesized that there would be a significant relationship between greater actor positive dyadic coping and less actor negative dyadic coping and greater partner health-related quality of life. Positive and negative dyadic coping were examined in separate analyses for each of physical, 
social, emotional, and functional well-being. The results of the APIM analyses for positive and negative dyadic coping are displayed in tables 7 and 8 , respectively.

Physical well-being. Employment, current treatment, and treatment progress were included as covariates for analyses of physical well-being. The interaction model for positive dyadic coping revealed significant main effects for role, $b=-1.23, p<.001$ and employment, $b=$ $-0.49, p<.05$. Correcting for multiple analyses, only role remained significant. These results suggest that being a patient was associated with less physical well-being. The actor and partner effects and the interactions were not significant. As the interaction terms were not significant, a two-intercept model was not completed. The pseudo $R^{2}$ for the present model was 0.216 ; that is, the effects of role, employment, current treatment, treatment progress, actor positive dyadic coping, and partner positive dyadic coping accounted for $21.6 \%$ of the variance in physical wellbeing.

The interaction model for negative dyadic coping revealed significant main effects for role, $b=1.88, p<.001$ and employment, $b=-0.61, p<.05$. Correcting for multiple analyses, only role remained significant. These results suggest that being a patient was associated with less physical well-being. The actor, partner, and interaction effects were not significant. As the interaction effects were non-significant, a two-intercept model was not completed. The pseudo $R^{2}$ for the present model was 0.220 ; that is, the effects of role, family history of cancer, employment, actor negative dyadic coping, and partner negative dyadic coping accounted for $22 \%$ of the variance in physical well-being.

Social well-being. Age, relationship duration, and employment were entered as covariates for the analyses of social well-being. The interaction model for positive dyadic coping revealed significant main effects for role, $b=-1.68, p<.001$, age, $b=0.09, p<.05$, and actor 
Table 7.

APIM Interaction Model Estimating Effects of Positive Dyadic Coping on Quality of Life

\begin{tabular}{|c|c|c|c|c|c|c|c|c|}
\hline & PWB & & SWB & & EWB & & FWB & \\
\hline Predictors & $b$ & $S E$ & $b$ & $S E$ & $b$ & $S E$ & $b$ & $S E$ \\
\hline Age & - & - & $.09 *$ & .04 & - & - & $.10 *$ & .04 \\
\hline Employment & $-.63 *$ & .25 & -.49 & .29 & $-.74 * *$ & .28 & $-1.57 * * *$ & .36 \\
\hline Current treatment & .76 & .48 & - & - & - & - & - & - \\
\hline Partner positive dyadic coping & .89 & .67 & .15 & .57 & -.09 & .60 & -.38 & .67 \\
\hline Role*Actor positive dyadic coping & -.01 & .62 & .39 & .61 & -.10 & .60 & 1.17 & .75 \\
\hline Role*Partner positive dyadic coping & -.44 & .66 & -.80 & .59 & -.81 & .62 & -1.38 & .75 \\
\hline
\end{tabular}

$* p<.05, * * p<.01, * * * p<.001$ 
Table 8 .

APIM Interaction Model Estimating Effects of Negative Dyadic Coping on Quality of Life

\begin{tabular}{|c|c|c|c|c|c|c|c|c|}
\hline & PWB & & SWB & & EWB & & FWB & \\
\hline Predictors & $b$ & $S E$ & $b$ & $S E$ & $\boldsymbol{b}$ & $S E$ & $\boldsymbol{b}$ & $S E$ \\
\hline Role & $1.88 * * *$ & .35 & $-1.85 * * *$ & .34 & $1.65 * * *$ & .31 & $.74 *$ & .34 \\
\hline Age & - & - & .05 & .05 & - & - & $.10^{*}$ & .04 \\
\hline Relationship duration & - & - & $.08^{*}$ & .03 & - & - & - & - \\
\hline Employment & $-.61^{*}$ & .25 & -.33 & .33 & $-.72 *$ & .29 & $-1.55^{* * *}$ & .37 \\
\hline Current treatment & .79 & .50 & - & - & - & - & - & - \\
\hline Treatment progress & .45 & .37 & - & - & - & - & - & - \\
\hline Actor negative dyadic coping & -1.38 & .81 & $-3.39 * * *$ & .80 & -1.11 & .74 & $-2.40^{* *}$ & .84 \\
\hline Partner negative dyadic coping & .42 & .84 & .02 & .79 & .23 & .75 & .61 & .84 \\
\hline Role*Actor negative dyadic coping & .60 & .80 & 1.30 & .89 & .94 & .79 & .36 & 1.03 \\
\hline Role*Partner negative dyadic coping & -.41 & .84 & -.67 & .88 & -.24 & .79 & .55 & 1.03 \\
\hline
\end{tabular}

$* p<.05, * * p<.01, * * * p<.001$ 
positive dyadic coping, $b=4.79, p<.001$. Correcting for multiple analyses, role and actor positive dyadic coping remained significant. These results suggest that being a patient was associated with better social well-being. Additionally, the main actor effect suggests that an individual's own greater positive dyadic coping was associated with one's own better social well-being. The partner and interaction effects were not significant. As the interaction effects were not significant, a two-intercept model was not completed. The pseudo $R^{2}$ for this model was 0.374 ; that is, the effects of role, age, relationship duration, family history, actor positive dyadic coping, and partner positive dyadic coping accounted for $37.4 \%$ of the variance in social wellbeing.

The interaction model for negative dyadic coping revealed significant main effects for role $b=-1.85, p<.001$, relationship duration, $b=0.08, p<.05$, and actor negative dyadic coping $b=-3.39, p<.001$. Correcting for multiple analyses, role and actor negative dyadic coping remained significant. These results suggest that being a patient was associated with better social well-being. The significant actor effect suggests that an individual's own greater negative dyadic coping was associated with one's own less social well-being. The partner and interaction effects were not significant. As the interaction effects were not significant, a two-intercept model was not completed. The pseudo $R^{2}$ for the present model was 0.217 ; that is, the effects of role, age, employment, relationship duration, actor negative dyadic coping, and partner negative dyadic coping accounted for $21.7 \%$ of the variance in social well-being.

Emotional well-being. Employment was entered as a covariate for analyses of emotional well-being. The interaction model for positive dyadic coping revealed significant main effects for role, $b=1.75, p<.001$, employment, $b=-0.74, p<.01$, and actor positive dyadic coping, $b=$ $1.15, p<.05$. Correcting for multiple analyses, the actor effect of positive dyadic coping was no 
longer significant. These results suggest that being a patient and less involvement in the workforce were associated with worse emotional well-being. The partner and interaction effects were not significant, therefore a two-intercept model was not completed. The pseudo $R^{2}$ for the present model was 0.184 ; that is, the effects of role, employment, actor positive dyadic coping, and partner positive dyadic coping accounted for $18.4 \%$ of the variance in emotional well-being.

The interaction model for negative dyadic coping revealed significant main effects of role, $b=1.65, p<.001$, and employment, $b=-0.72, p<.05$. Correcting for multiple analyses, only role remained significant. These results suggest that being a patient was associated with less emotional well-being. The actor and partner effects as well as the interaction effects were nonsignificant. As the interaction effects were not significant, a two-intercept model was not completed. The pseudo $R^{2}$ for the present model was 0.174 ; that is, the effects of role, employment, actor negative dyadic coping, and partner negative dyadic coping accounted for $17.4 \%$ of the variance in emotional well-being.

Functional well-being. Age and employment were entered as covariates for analyses of functional well-being. The interaction model for positive dyadic coping revealed significant main effects for role, $b=0.95, p<.01$, age, $b=0.10, p<.05$, employment, $b=-1.57, p<.001$, and actor positive dyadic coping, $b=2.91, p<.001$. Correcting for multiple analyses, role, employment, and actor positive dyadic coping remained significant. These results suggest that being a patient and less involvement in the workforce were associated with less functional wellbeing. Additionally, the significant actor effect suggests that an individual's own greater positive dyadic coping was associated with one's own greater functional well-being. The partner and interaction effects were not significant. As the interaction effect was not significant, a twointercept model was not completed. The pseudo $R^{2}$ for this model was 0.096; that is, the effect of 
role, employment, actor positive dyadic coping, and partner positive dyadic coping accounted for $9.6 \%$ of the variance in functional well-being.

The interaction model for negative dyadic coping revealed significant main effects for role, $b=0.74, p<.05$, age, $b=0.10, p<.05$ employment, $b=-1.55, p<.001$, and actor negative dyadic coping $b=-2.40, p<.01$. Correcting for multiple analyses, employment and actor negative dyadic coping remained significant. These results suggest that less involvement in the workforce was associated with less functional well-being. Additionally, the significant actor effect suggests that an individual's own greater negative dyadic coping was associated with one's own less functional well-being. The partner and interaction effects were not significant, therefore a two-intercept model was not completed. The pseudo $R^{2}$ for this model was 0.132 ; that is, the effects of role, age, employment, actor negative dyadic coping, and partner negative dyadic coping accounted for $13.2 \%$ of the variance in functional well-being.

Summary. In sum, there were significant actor effects of positive dyadic coping for social well-being and functional well-being. The actor effects of positive dyadic coping for physical and emotional well-being were not significant. There were also significant actor effects of negative dyadic coping for social and functional well-being. The actor effects of negative dyadic coping for physical and emotional well-being were not significant. There was no evidence of significant partner effects nor was there evidence that the magnitude of the actor or partner effects differed for patients compared to spouses. These models accounted for between $10-37 \%$ of the variance in well-being.

\section{Aim 3: To Examine Dyadic Coping as a Mediator Between Attachment and Quality of Life}

It was hypothesized that dyadic coping would mediate the actor and partner effects of attachment on quality of life. Anxious and avoidant attachment as predictors, as well as positive 
and negative dyadic coping as mediators, were each examined in separate analyses to determine their effects on each of physical, social, emotional, and functional well-being. As there was no evidence of moderation by role in any of the above analyses, the meditational effects were averaged across dyad members. Multilevel models (MLMs) were completed for each effect and mediation was assessed using a Sobel test.

Physical well-being. Employment, current treatment, and treatment progress were included as covariates for the analyses of physical well-being. They were entered as fixed effects in each of the MLM analyses.

Positive dyadic coping. The results of the model examining positive dyadic coping as a mediator of the effect of avoidant attachment on physical well-being are displayed in Figure $7^{1}$. There were significant actor, $b=-0.22, p<.001$, and partner, $b=-0.07, p=.02$, effects of avoidant attachment on positive dyadic coping. There was also a significant direct actor effect, $b$ $=-0.65, p=.02$, of avoidant attachment on physical well-being. Correcting for multiple analyses, only the actor effect of avoidant attachment on positive dyadic coping remained significant. That is, one's own greater avoidant attachment was associated with one's own less positive dyadic coping. There were no significant indirect effects.

The results of the model examining positive dyadic coping as a mediator of the effect of anxious attachment on physical well-being are displayed in Figure 8. There were significant actor, $b=-0.14, p<.001$, and partner, $b=-0.14, p<.001$, effects of anxious attachment on positive dyadic coping. That is, one's own greater anxious attachment and one's partner's greater anxious attachment were associated with one's own less positive dyadic coping. There was also a

1 The dashed lines indicate significant direct and indirect effects of the mediation model. Solid lines indicate the individual effects that were evaluated within the model. Although some of the solid lines may themselves be significant, the indirect pathway did not reach overall significance. This applies throughout Aim 3. 


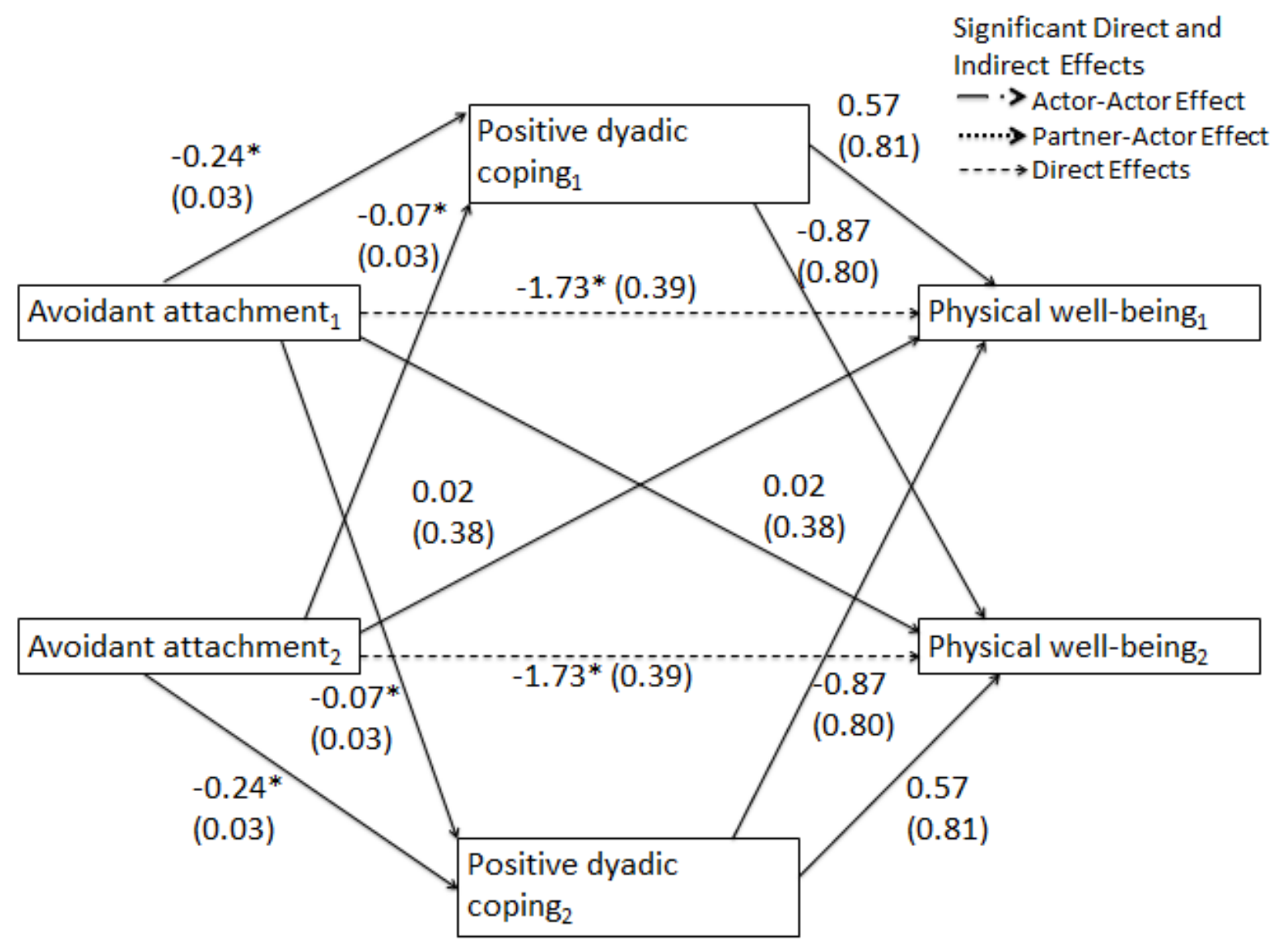

Figure 7. Positive dyadic coping as a mediator for avoidant attachment and physical well-being. Note. Dashed lines indicate specific aspects of mediation such as actor-actor mediation and direct effects. Solid lines denote each pathway that was evaluated.

1 and ${ }_{2}$ refer to different members of the dyad; $" p<.05$ 


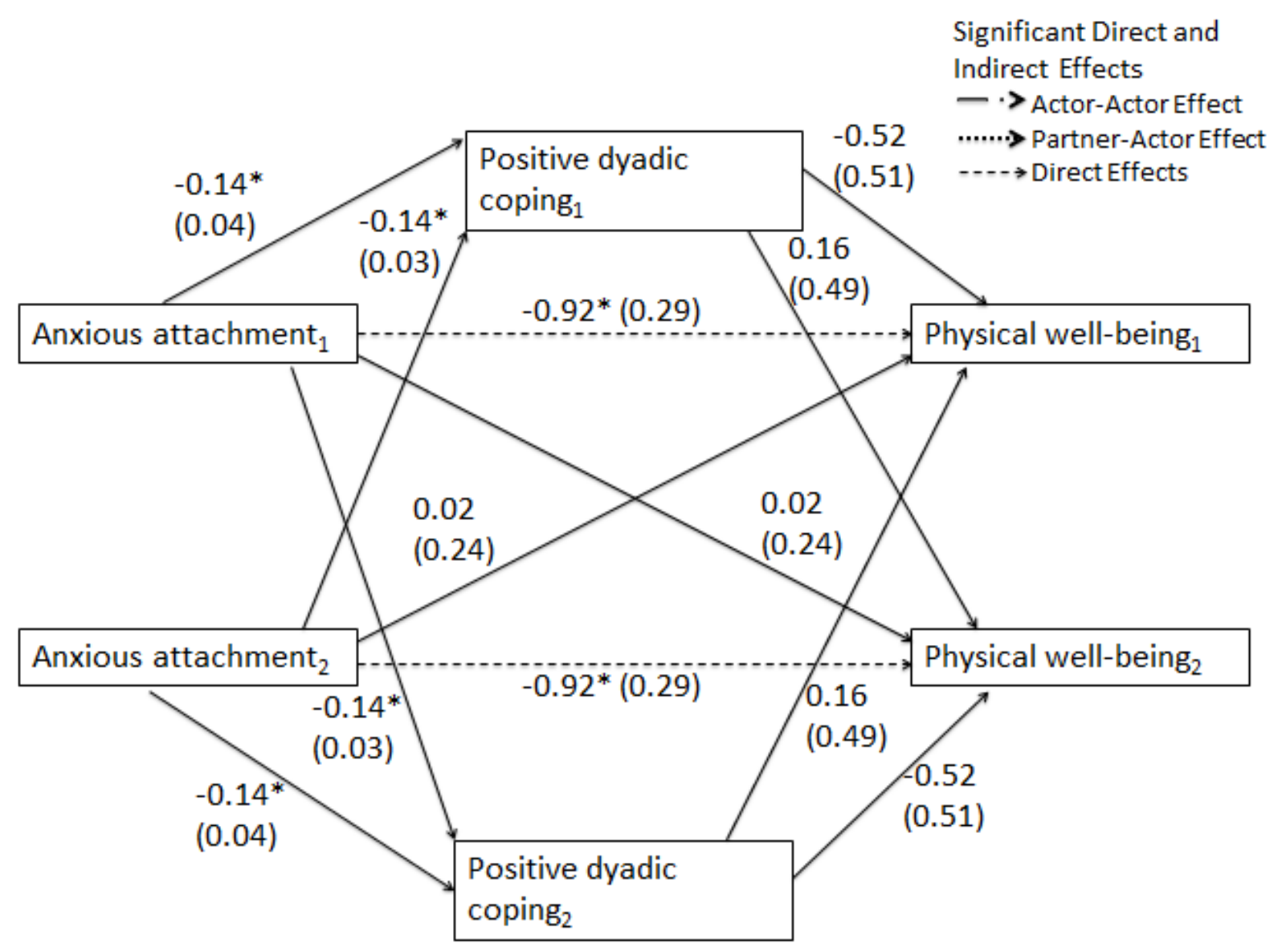

Figure 8. Positive dyadic coping as a mediator for anxious attachment and physical well-being. Note. Dashed lines indicate specific aspects of mediation such as actor-actor mediation and direct effects. Solid lines denote each pathway that was evaluated.

1 and ${ }_{2}$ refer to different members of the dyad; $" p<.05$ 
significant direct actor effect, $b=-0.92, p=.002$, of anxious attachment on physical well-being, such that greater anxious attachment was associated with one's own worse physical well-being. Correcting for multiple analyses, all of these effects remained significant. There were no significant indirect effects.

Negative dyadic coping. The results of the model examining negative dyadic coping as a mediator of the effect of avoidant attachment on physical well-being are displayed in Figure 9. There was a significant actor effect, $b=0.09, p=.003$, of avoidant attachment on negative dyadic coping. This effect remained significant after correcting for multiple analyses. That is, one's own greater avoidant attachment was associated with one's own greater negative dyadic coping. There were no significant indirect effects.

The results of the model examining negative dyadic coping as a mediator of the effect of anxious attachment on physical well-being are displayed in Figure 10. There were significant actor, $b=0.13, p<.001$, and partner, $b=0.10, p=.001$, effects of anxious attachment on negative dyadic coping. That is, one's own greater anxious attachment and one's partner's greater anxious attachment were associated with one's own greater negative dyadic coping. There was also a significant direct actor effect, $b=-0.81, p=.006$, of anxious attachment on physical well-being, such that greater anxious attachment was associated with one's own worse physical well-being. These effects remained significant after correcting for multiple analyses. There were no significant indirect effects.

Social well-being. Age, relationship duration, and employment were included as covariates for analyses of social well-being. They were entered as fixed effects for each of the MLMs. 


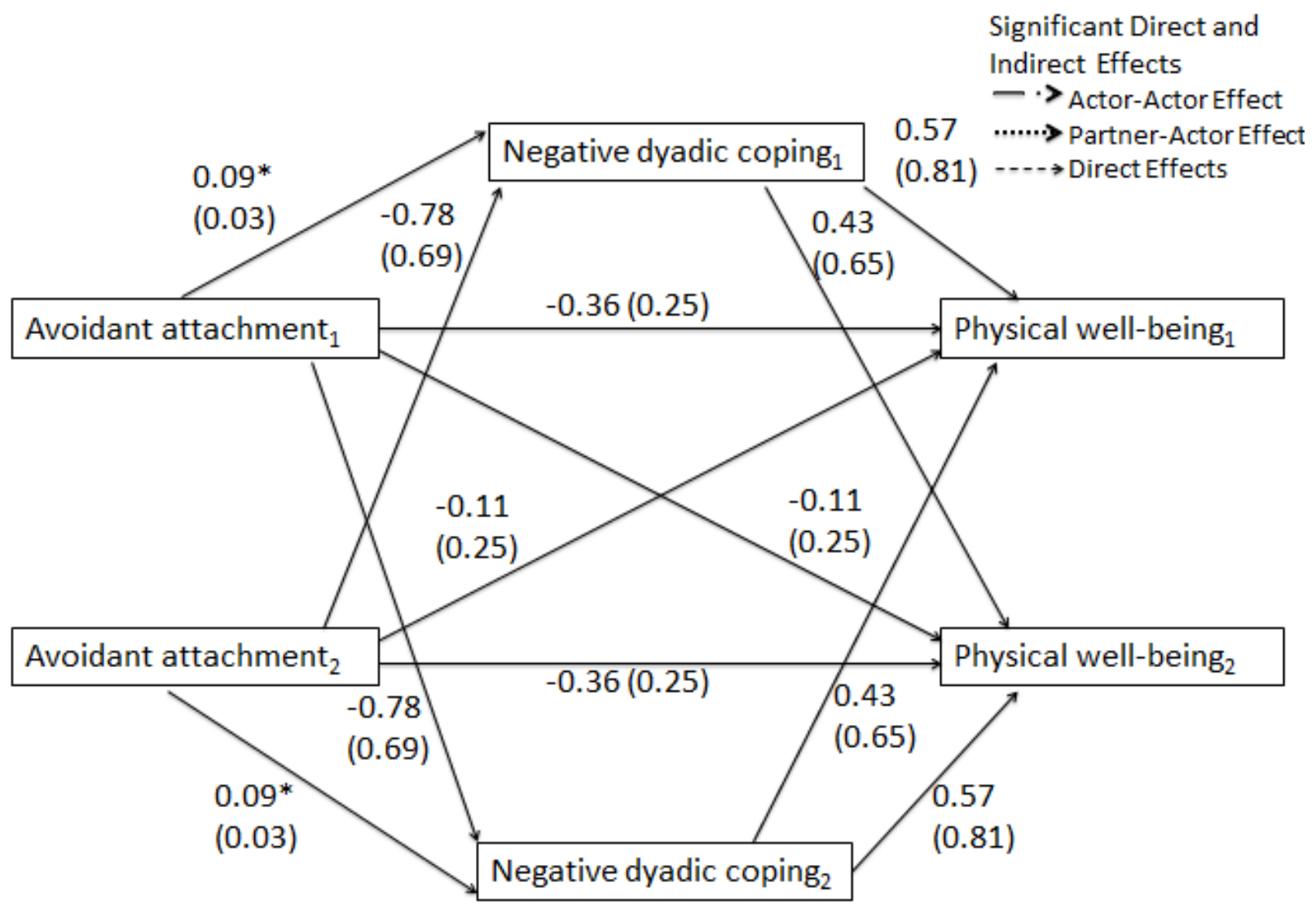

Figure 9. Negative dyadic coping as a mediator for avoidant attachment and physical well-being. Note. Dashed lines indicate specific aspects of mediation such as actor-actor mediation and direct effects. Solid lines denote each pathway that was evaluated.

$I_{1}$ and ${ }_{2}$ refer to different members of the dyad; $" p<.05$ 


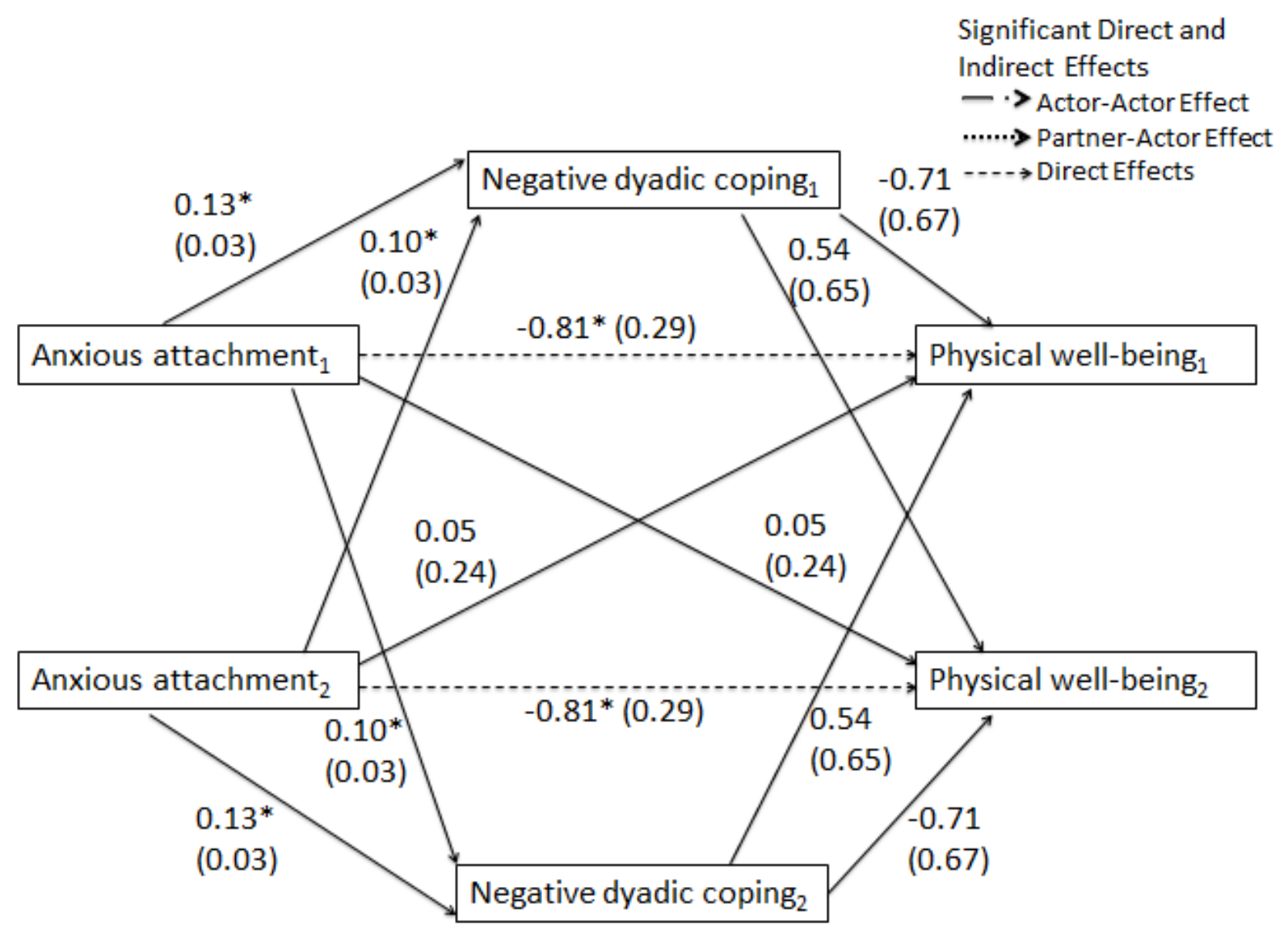

Figure 10. Negative dyadic coping as a mediator for anxious attachment and physical well-being. Note. Dashed lines indicate specific aspects of mediation such as actor-actor mediation and direct effects. Solid lines denote each pathway that was evaluated.

${ }_{1}$ and ${ }_{2}$ refer to different members of the dyad; $* 0<.05$ 
Positive dyadic coping. The results of the model examining positive dyadic coping as a mediator of the effect of avoidant attachment on social well-being are displayed in Figure 11. There were significant actor, $b=-0.24, p<.001$, and partner, $b=-0.07, p=.015$, effects of avoidant attachment on positive dyadic coping. Correcting for multiple analyses, the partner of effect of avoidant attachment on positive dyadic coping was no longer significant. One's own greater avoidant attachment was associated with one's own less positive dyadic coping. There was a significant actor effect, $b=3.75, p<.001$, of positive dyadic coping on social well-being, such that one's own greater positive dyadic coping was associated with one's own greater social well- being. The direct actor effect of avoidant attachment on social well-being was significant, $b$ $=-1.26, p<.001$, such that one's own greater avoidant attachment was associated with one's own less social well-being. This direct effect remained significant after correcting for multiple analyses.

The significant indirect effects are highlighted in Figure 11. The actor-actor indirect effect was significant, $b=-0.91, p<.001$. That is, the effect of one's own greater avoidant attachment on one's own worse social well-being was significantly mediated by less positive dyadic coping, accounting for $40.52 \%$ of the total actor effect. The partner-actor indirect effect was also significant, $b=-0.27, p=.025$. Correcting for multiple analyses, the partner-actor indirect effect was no longer significant.

The results of the model examining positive dyadic coping as a mediator of the effect of anxious attachment on social well-being are displayed in Figure 12. There were significant actor, $b=-0.15, p<.001$, and partner, $b=-0.12, p<.001$, effects of anxious attachment on positive dyadic coping. That is, one's own greater anxious attachment and one's partner's greater anxious attachment were associated with one's own less positive dyadic coping. There was a significant 


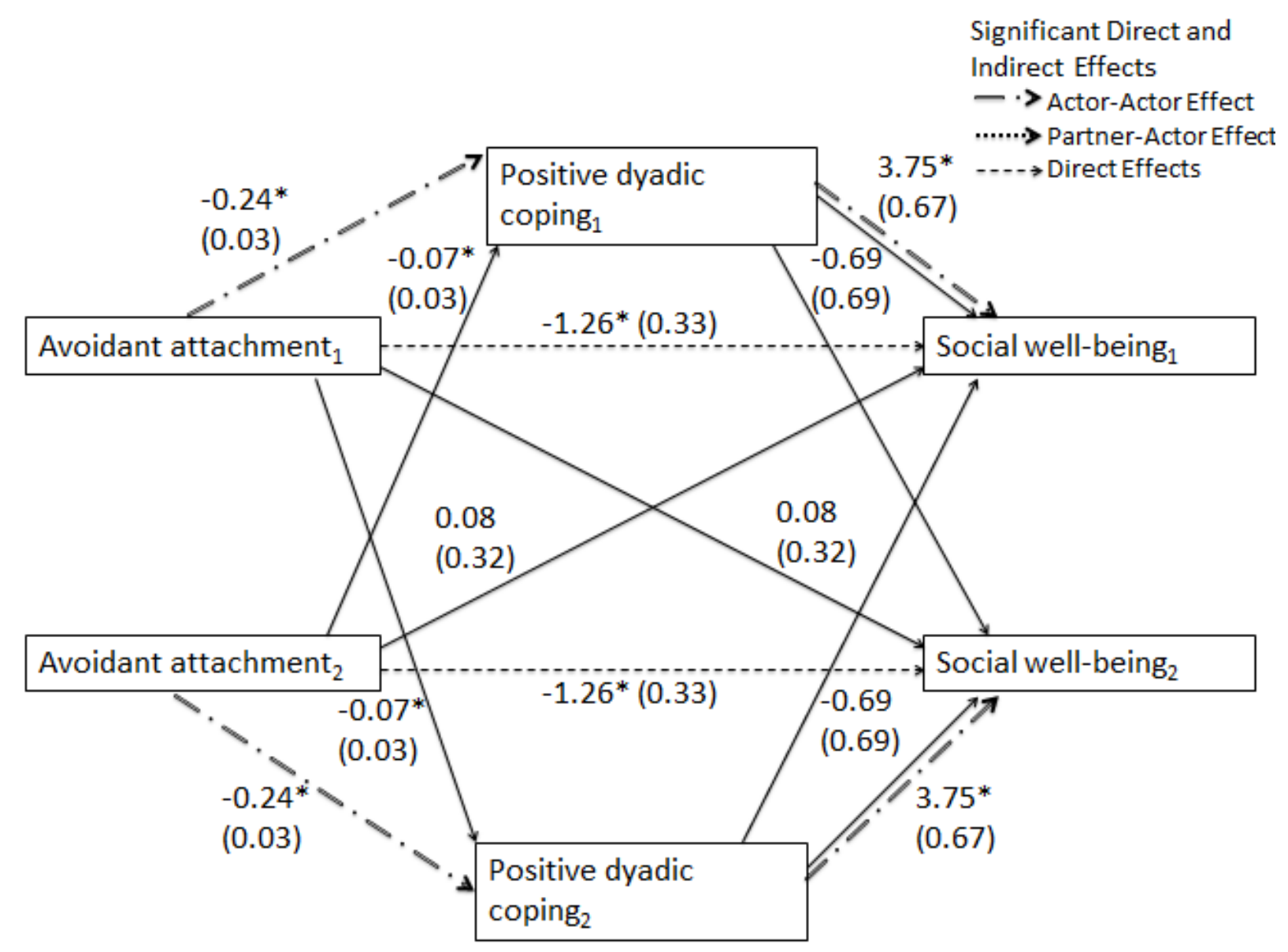

Figure 11. Positive dyadic coping as a mediator for avoidant attachment and social well-being. Note. Dashed lines indicate specific aspects of mediation such as actor-actor mediation and direct effects. Solid lines denote each pathway that was evaluated.

${ }_{1}$ and $_{2}$ refer to different members of the dyad; $* 0<.05$ 


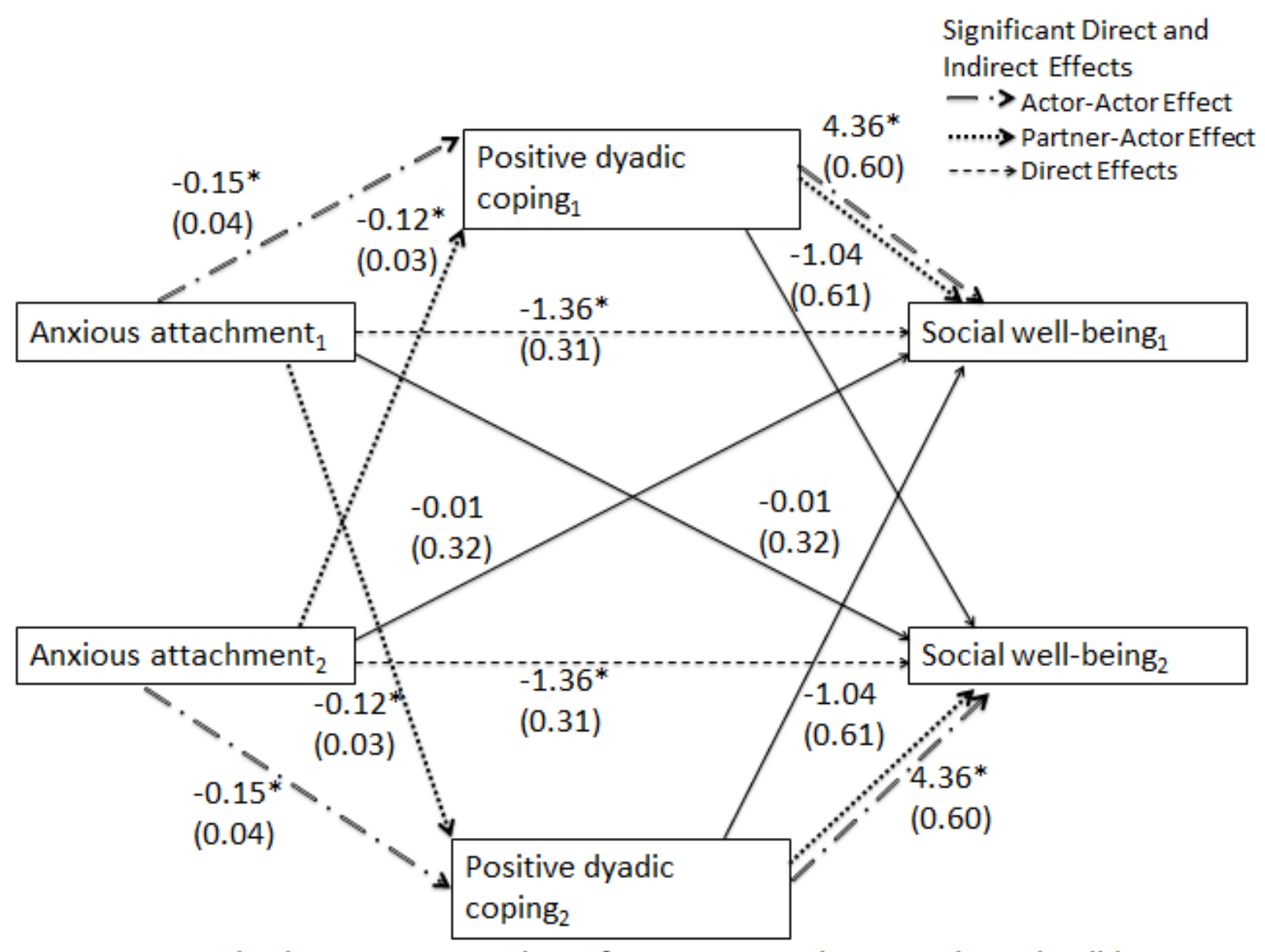

Figure 12. Positive dyadic coping as a mediator for anxious attachment and social well-being. Note. Note. Dashed lines indicate specific aspects of mediation such as actor-actor mediation and direct effects. Solid lines denote each pathway that was evaluated.

${ }_{1}$ and ${ }_{2}$ refer to different members of the dyad; $" p<.05$ 
actor effect, $b=4.36, p<.001$, of positive dyadic coping on social well-being, such that one's own greater positive dyadic coping was associated with one's own greater social well-being. The direct actor effect of anxious attachment on social well-being was significant, $b=-1.36, p<$ .001 , such that one's own greater anxious attachment was associated with one's own less social well-being. Each of these effects remained significant after correcting for multiple analyses.

The significant indirect effects are highlighted in Figure 12. The actor-actor indirect effect was significant, $b=-0.68, p<.001$. That is, the effect of one's own greater anxious attachment on one's own less social well-being was significantly mediated by one's own less positive dyadic coping, accounting for $28.71 \%$ of the total actor effect. The partner-actor indirect effect was also significant, $b=-0.54, p=.001$. That is, one's partner's greater anxious attachment was significantly associated with one's own less positive dyadic coping, which was associated with less social well-being. Each of these indirect effects remained significant after correcting for multiple analyses. As the total partner effect was not significant, an estimate of the amount of the total partner effect accounted for cannot be meaningfully interpreted ${ }^{2}$.

Negative dyadic coping. The results of the model examining negative dyadic coping as a mediator of the effect of avoidant attachment on social well-being are displayed in Figure 13. There was a significant actor effect of avoidant attachment on negative dyadic coping, $b=0.10$, $p<.001$, such that one's own greater avoidant attachment was associated with one's own greater negative dyadic coping. There was also a significant actor effect of negative dyadic coping on social well-being, $b=-2.52, p=.002$, such that one's own greater negative dyadic coping was associated with one's own worse social well-being. The direct actor effect of avoidant

${ }^{2}$ Total effects are comprised of direct and indirect effects. As the direct partner effects were not significant for any of the analyses, the total partner effects were non-significant. As per personal communication with David A. Kenny, without a significant total effect, the percent of variance accounted for by the indirect effect cannot be meaningfully calculated or interpreted. 


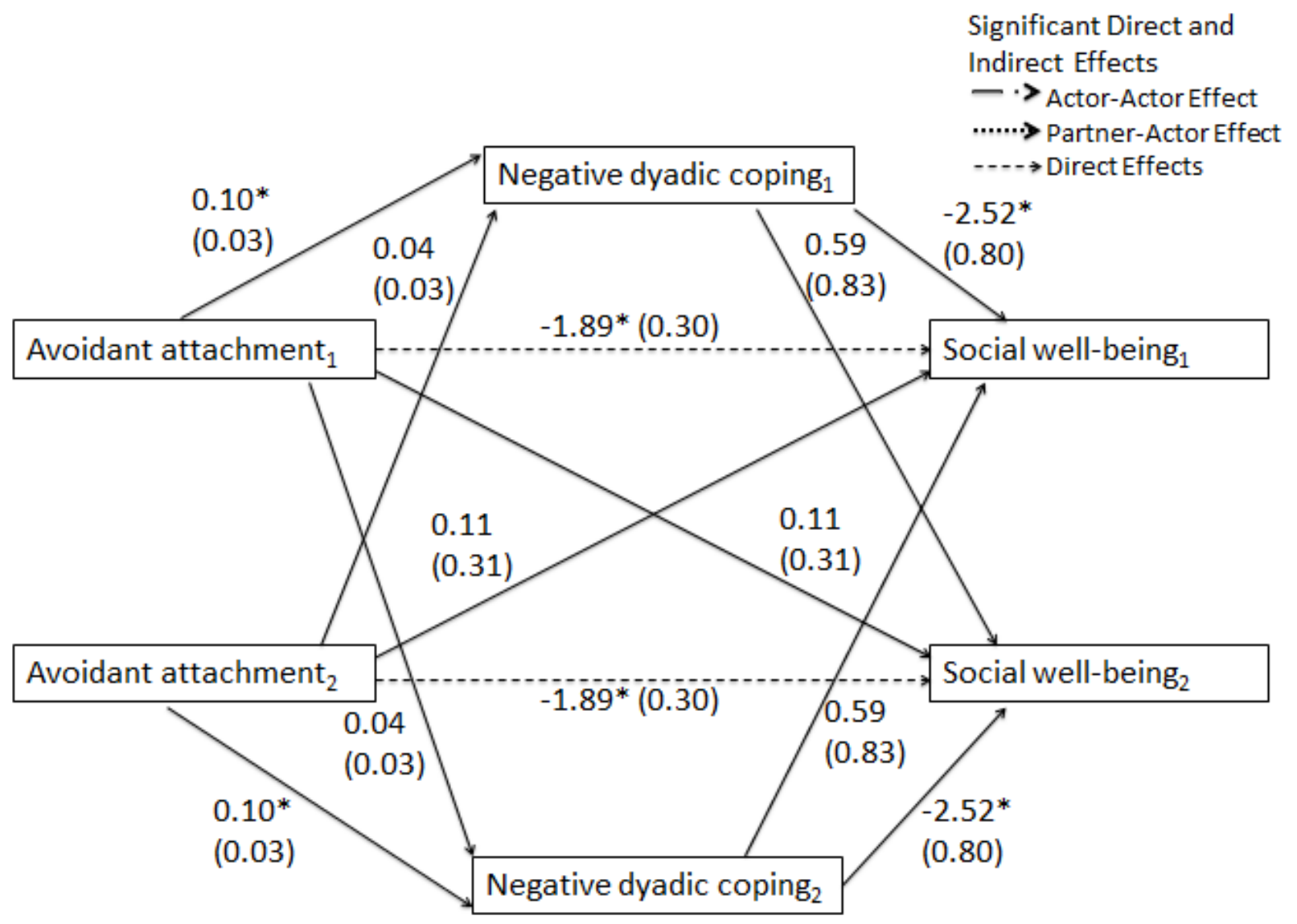

Figure 13. Negative dyadic coping as a mediator for avoidant attachment and social well-being. Note. Dashed lines indicate specific aspects of mediation such as actor-actor medizion and direct effects. Solid lines denote each pathway that was evaluated.

${ }_{1}$ and ${ }_{2}$ refer to different members of the dyad; $* 0<.05$ 
attachment on social well-being was significant, $b=-1.89, p<.001$, such that one's own greater avoidant attachment was associated with one's own less social well-being. Each of these effects remained significant after correcting for multiple analyses.

The actor-actor indirect effect, highlighted in Figure 13, was significant, $b=-0.26, p=$ .017. The actor-actor indirect effect was no longer significant after correcting for multiple analyses.

The results of the model examining negative dyadic coping as a mediator of the effect of anxious attachment on social well-being is displayed in Figure 14. There were significant actor, $b$ $=0.14, p<.001$, and partner, $b=0.09, p=.002$, effects of anxious attachment on negative dyadic coping. That is, one's own greater anxious attachment and one's partner's greater anxious attachment were associated with one's own greater negative dyadic coping. There was a significant actor effect, $b=-2.68, p=.002$, of negative dyadic coping on social well-being, such that one's own greater negative dyadic coping was associated with one's own worse social wellbeing. The direct actor effect of anxious attachment on social well-being was significant, $b=-$ $1.62, p<.001$, such that one's own greater anxious attachment was associated with one's own less social well-being. Each of these effects remained significant after correcting for multiple analyses.

The significant indirect effects are highlighted in Figure 14. The actor-actor indirect effect was significant, $b=-0.38, p=.008$. That is, one's own greater negative dyadic coping significantly mediated the association between greater anxious attachment and less social wellbeing, accounting for $15.29 \%$ of the total actor effect. The partner-actor indirect effect was also significant, $b=-0.25, p=.026$. The partner-actor indirect effect was no longer significant after correcting for multiple analyses. 


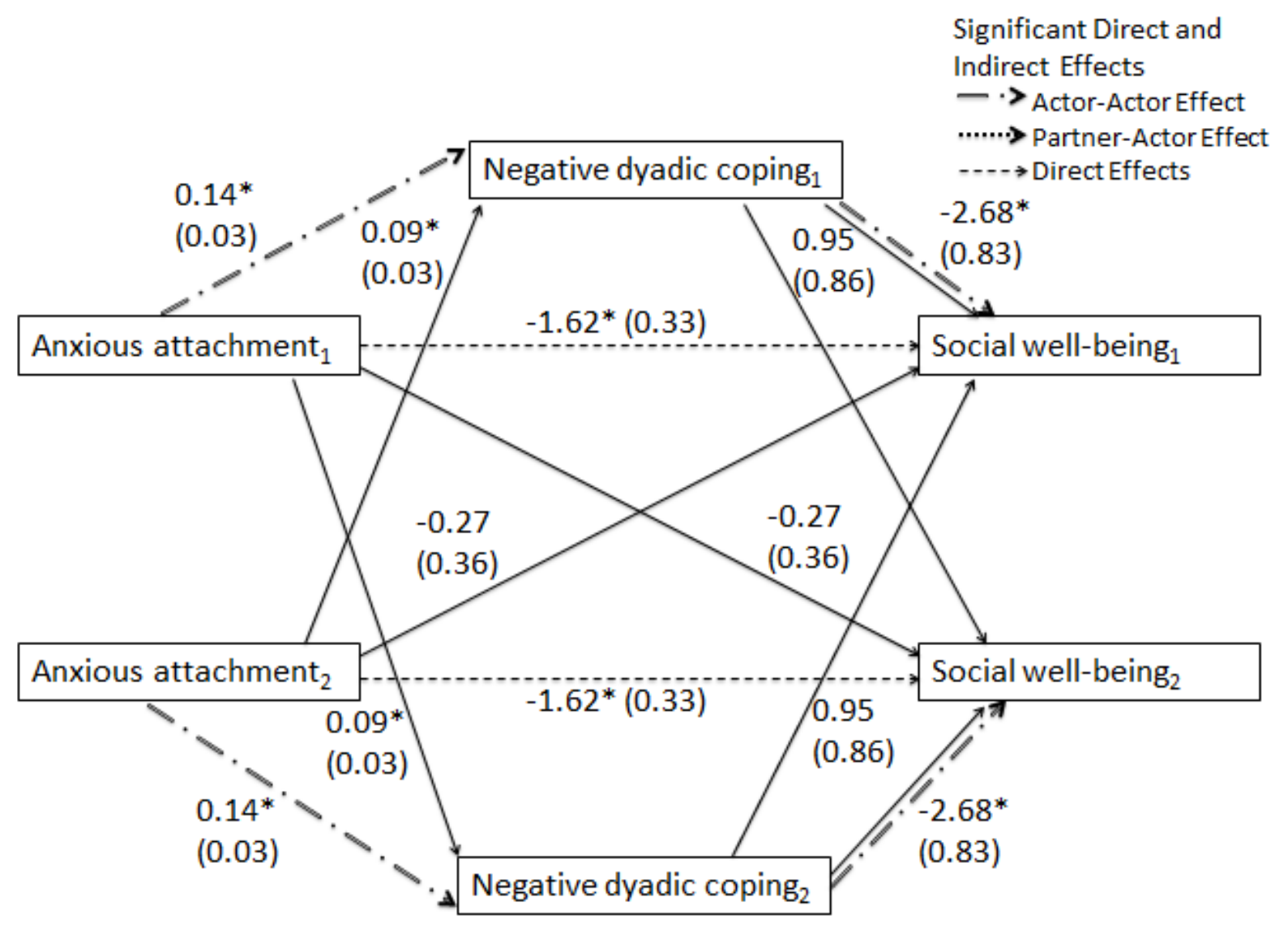

Figure 14. Negative dyadic coping as a mediator for anxious attachment and social well-being. Note. Dashed lines indicate specific aspects of mediation such as actor-actor mediation and direct effects. Solid lines denote each pathway that was evaluated.

${ }_{1}$ and $_{2}$ refer to different members of the dyad; $*<.05$ 
Emotional well-being. Employment was entered as a covariate. It was entered in each MLM as a fixed effect.

Positive dyadic coping. The results of the model examining positive dyadic coping as a mediator of the effect of avoidant attachment on emotional well-being are displayed in Figure 15. There were significant actor, $b=-0.24, p<.001$, and partner, $b=-0.08, p=.009$, effects of avoidant attachment on positive dyadic coping. That is one's own and one's partner's greater avoidant attachment was associated with one's own less positive dyadic coping. There was a significant direct actor effect of avoidant attachment on emotional well-being, $b=-1.14, p<$ .001 , such that greater avoidant attachment was associated with one's own worse emotional wellbeing. Each of these effects remained significant after correcting for multiple analyses. There were no significant indirect effects.

The results of the model examining positive dyadic coping as a mediator of the effect of anxious attachment on emotional well-being are displayed in Figure 16. There were significant actor, $b=-0.15, p<.001$, and partner, $b=-0.13, p<.001$, effects of anxious attachment on positive dyadic coping. That is, one's own and one's partner's greater anxious attachment was significantly associated with one's own less positive dyadic coping. There was a significant direct actor effect of anxious attachment on emotional well-being, $b=-1.19, p=.001$, such that one's own greater anxious attachment was associated with one's own less emotional well-being. Each of these effects remained significant after correcting for multiple analyses. There were no significant indirect effects.

Negative dyadic coping. The results of the model examining negative dyadic coping as a mediator of the effect of avoidant attachment on emotional well-being are displayed in Figure 17. There was a significant actor effect of avoidant attachment on negative dyadic coping, $b=$ 


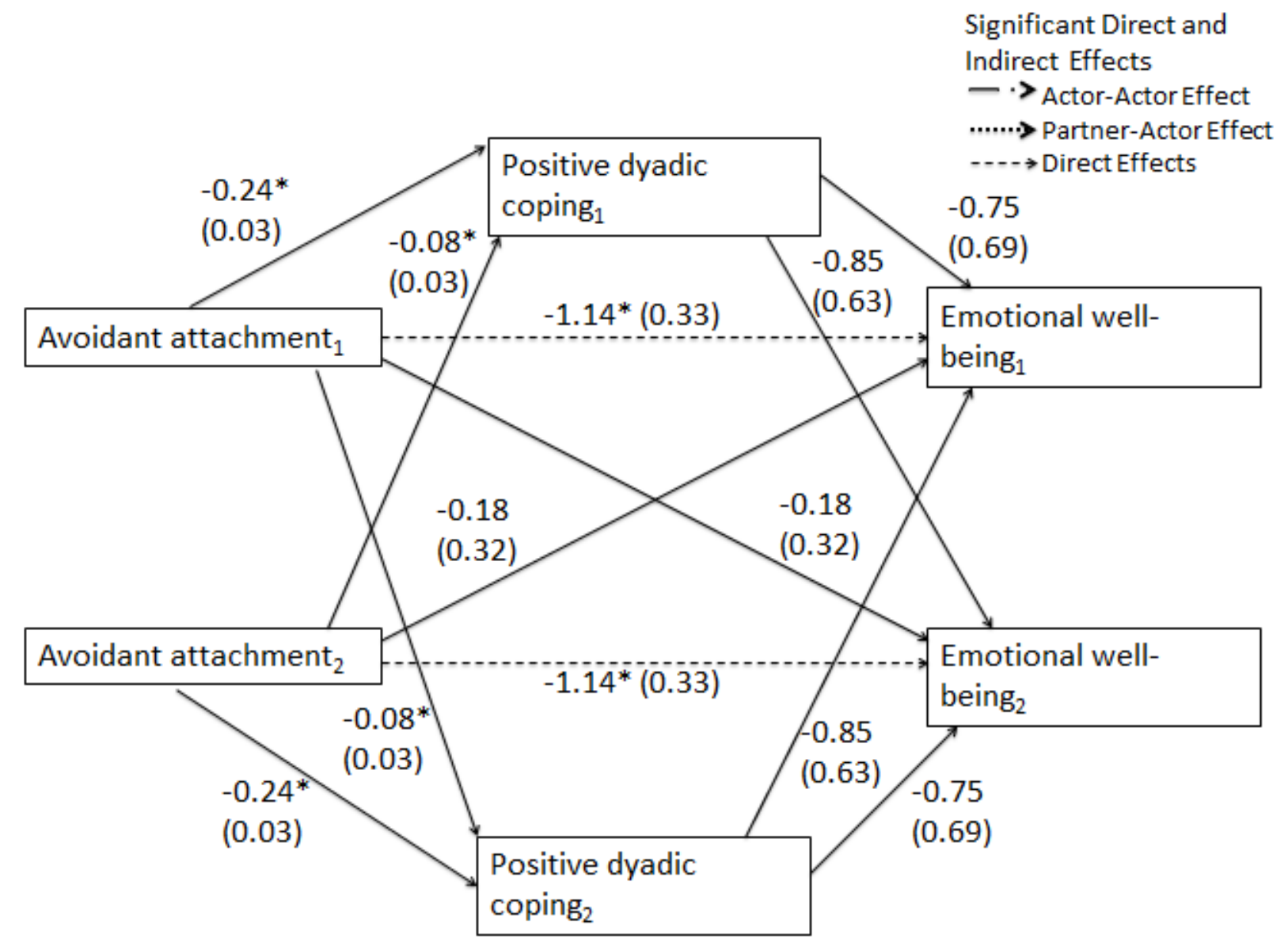

Figure 15. Positive dyadic coping as a mediator for avoidant attachment and emotional well-being. Note. Dashed lines indicate specific aspects of mediation such as actor-actor mediation and direct effects. Solid lines denote each pathway that was evaluated.

${ }_{1}$ and ${ }_{2}$ refer to different members of the dyad; $* 0<.05$ 


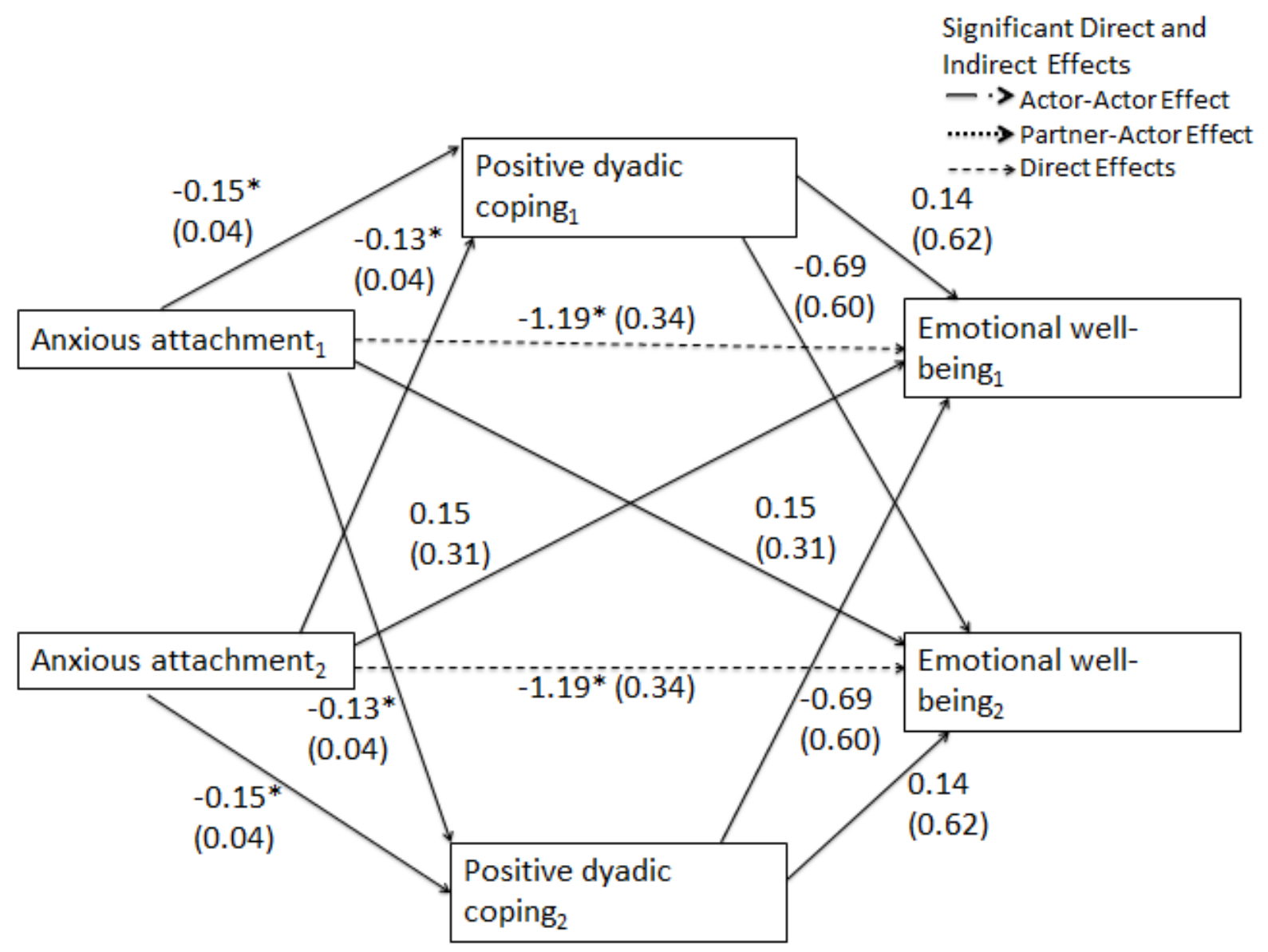

Figure 16. Positive dyadic coping as a mediator for anxious attachment and emotional well-being. Note. Dashed lines indicate specific aspects of mediation such as actor-actor mediation and direct effects. Solid lines denote each pathway that was evaluated.

${ }_{1}$ and $_{2}$ refer to different members of the dyad; $* 0.05$ 


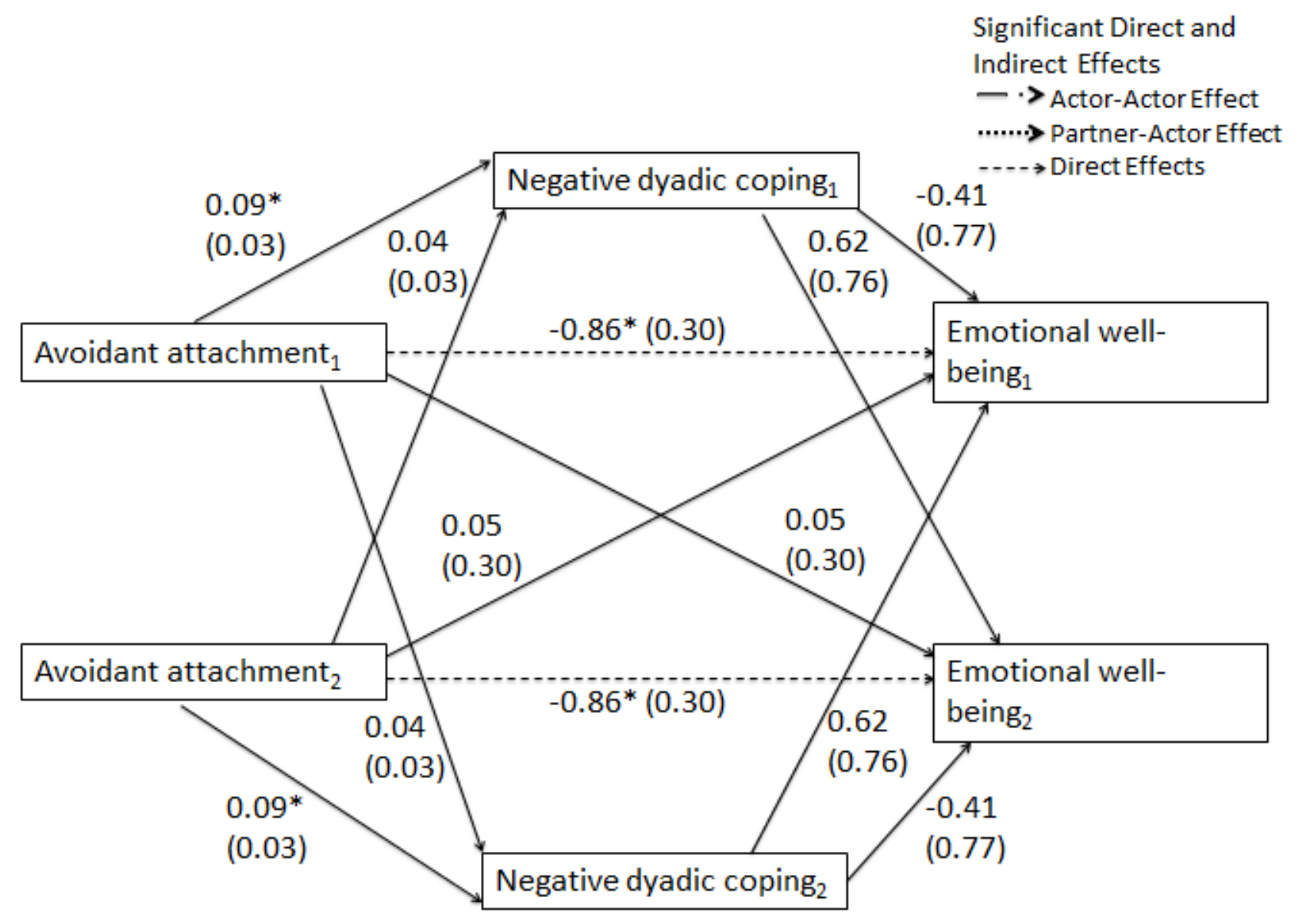

Figure 17. Negative dyadic coping as a mediator for avoidant attachment and emotional well-being. Note. Dashed lines indicate specific aspects of mediation such as actor-actor mediation and direct effects. Solid lines denote each pathway that was evaluated.

${ }_{1}$ and ${ }_{2}$ refer to different members of the dyad; $* 0<.05$ 
$0.09, p=.003$, such that one's own greater avoidant attachment was associated with one's own greater negative dyadic coping. There was also a significant direct effect of avoidant attachment on emotional well-being, $b=-0.86, p=.004$, such that one's own greater avoidant attachment was associated with one's own less emotional well-being. Each of these effects remained significant after correcting for multiple analyses. There were no significant indirect effects.

The results of the model examining negative dyadic coping as a mediator of the effect of anxious attachment on emotional well-being are displayed in Figure 18. There were significant actor, $b=0.13, p<.001$, and partner, $b=0.10, p=.002$, effects of anxious attachment on negative dyadic coping. That is, one's own and one's partner's greater anxious attachment was significantly associated with one's own less negative dyadic coping. There was also a significant direct actor effect of anxious attachment on emotional well-being, $b=-1.09, p=.001$, such that one's own greater anxious attachment was associated with one's own less emotional well-being. Each of these effects remained significant after applying correcting for multiple analyses. There were no significant indirect effects.

Functional well-being. Age and employment were entered as covariates. They were entered as fixed effects for each of the MLMs.

Positive dyadic coping. The results of the model examining positive dyadic coping as a mediator of the effect of avoidant attachment on functional well-being are displayed in Figure 19. There were significant actor, $b=-0.24, p<.001$, and partner, $b=-0.07, p=.015$, effects of avoidant attachment on positive dyadic coping. The partner effect of avoidant attachment on functional well-being was no longer significant after correcting for multiple analyses. One's own greater avoidant attachment was associated with one's own less positive dyadic coping. The direct actor effect of avoidant attachment on functional well-being was significant, $b=-1.73$, 


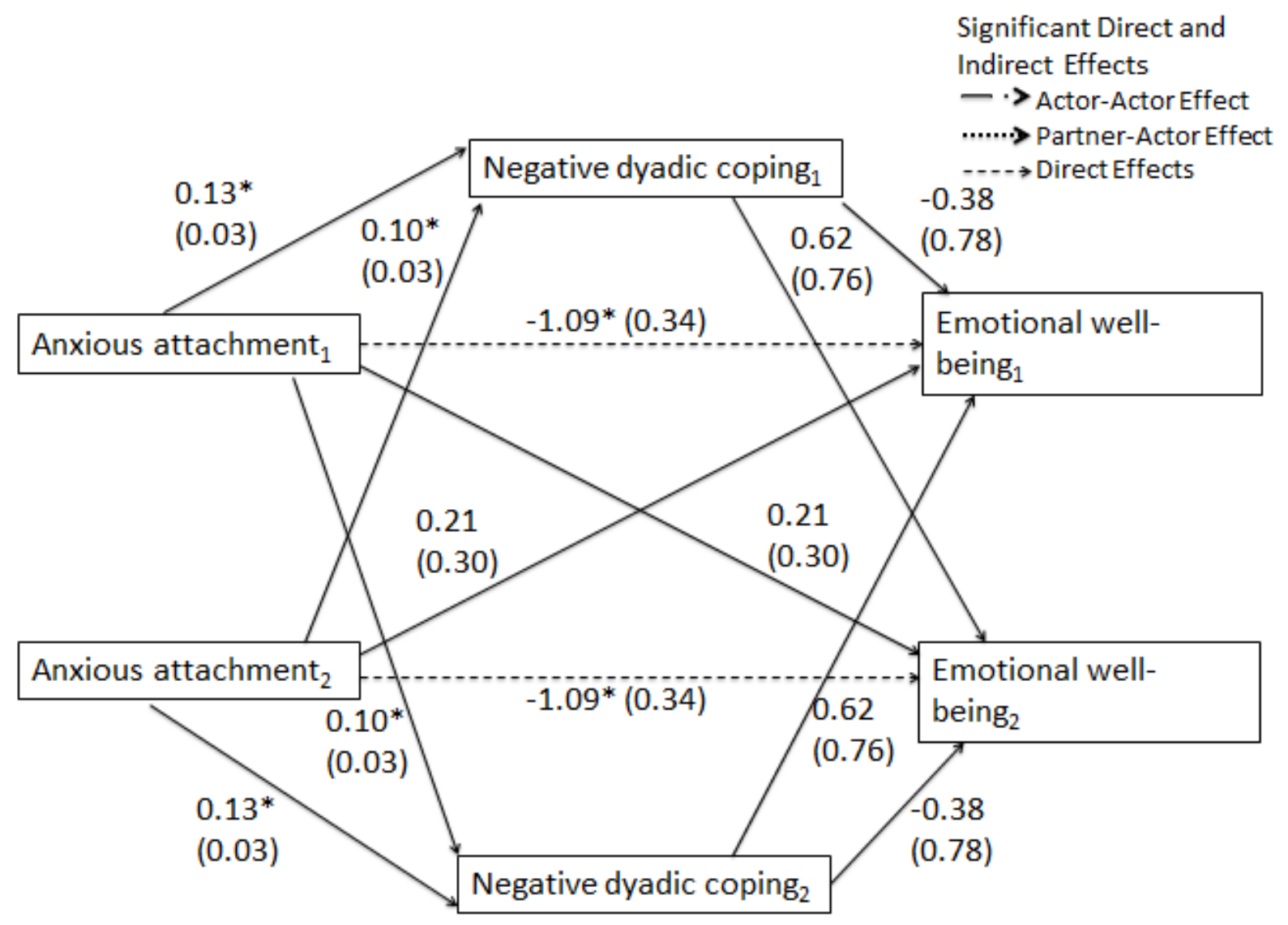

Figure 18. Negative dyadic coping as a mediator for anxious attachment and emotional well-being. Note. Dashed lines indicate specific aspects of mediation such as actor-actor mediation and direct effects. Solid lines denote each pathway that was evaluated.

${ }_{1}$ and $_{2}$ refer to different members of the dyad; $* 0<.05$ 


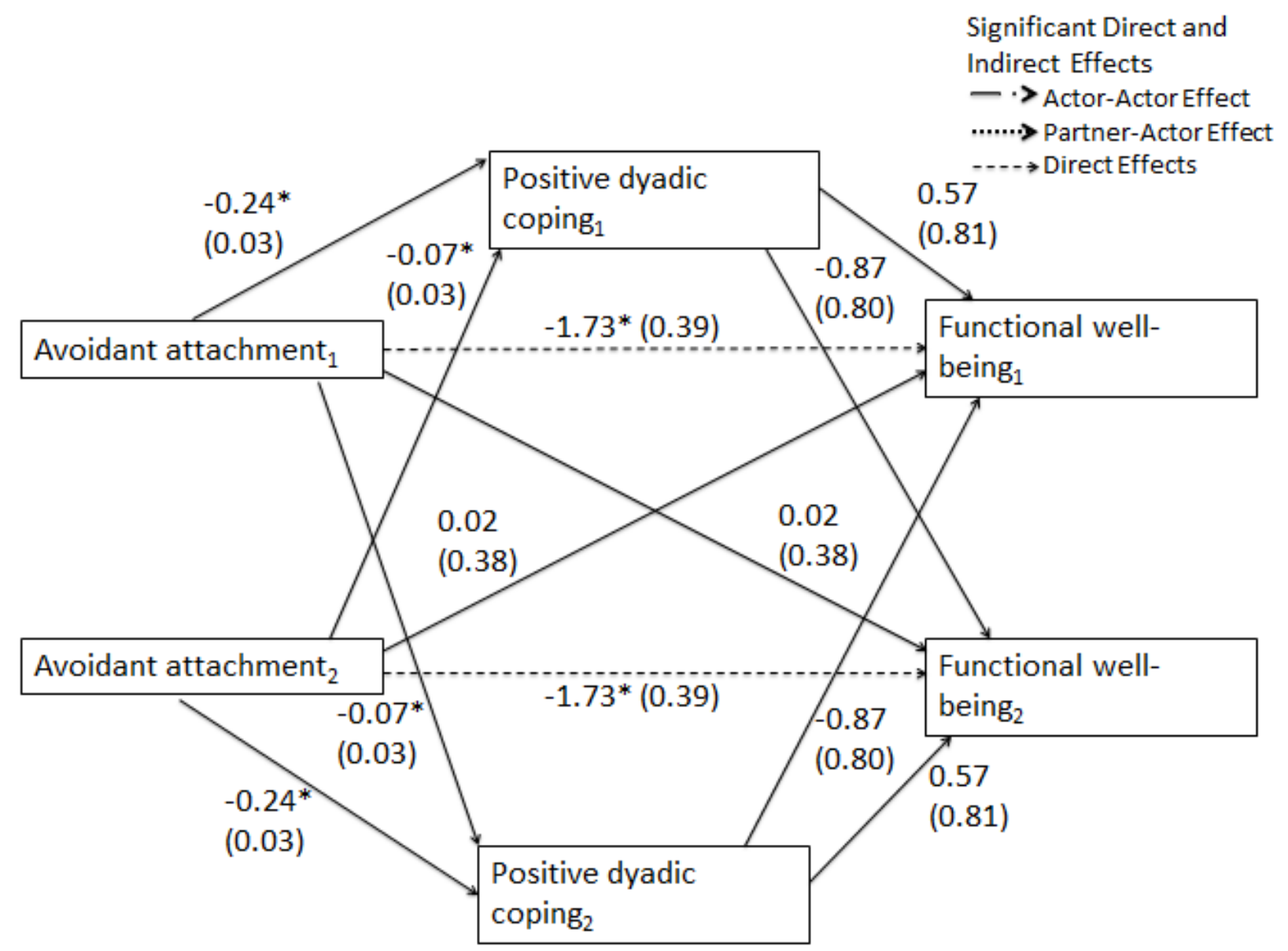

Figure 19. Positive dyadic coping as a mediator for avoidant attachment and functional well-being. Note. Dashed lines indicate specific aspects of mediation such as actor-actor mediation and direct effects. Solid lines denote each pathway that was evaluated.

${ }_{1}$ and ${ }_{2}$ refer to different members of the dyad; $" p<.05$ 
$p<.001$, such that one's own greater avoidant attachment was associated with one's own less functional well-being. There were no significant indirect effects.

The results of the model examining positive dyadic coping as a mediator of the effect of anxious attachment on functional well-being are displayed in Figure 20. There were significant actor, $b=-0.15, p<.001$, and partner, $b=-0.13, p<.001$, effects of anxious attachment on positive dyadic coping. That is, one's own and one's partner's greater anxious attachment was associated with one's own less positive dyadic coping. There was also a significant actor effect, $b=1.66, p=.025$, of positive dyadic coping on functional well-being. The significant actor effect of positive dyadic coping on functional well-being was no longer significant after applying the conservative $p$-value corrected for multiple analyses.

The direct actor effect of anxious attachment on functional well-being was significant, $b$ $=-1.58, p<.001$, such that one's own greater anxious attachment was associated with one's own less functional well-being. There was a significant actor-actor indirect effect, highlighted in Figure 20, $b=-0.25, p=.045$. The actor-actor indirect effect was no longer significant after using the correcting for multiple analyses.

Negative dyadic coping. The results of the model examining negative dyadic coping as a mediator of the effect of avoidant attachment on functional well-being are displayed in Figure 21. There was a significant actor effect of avoidant attachment on negative dyadic coping, $b=$ $0.10, p=.001$, such that one's own greater avoidant attachment was associated with one's own greater negative dyadic coping. The direct actor effect of avoidant attachment on functional wellbeing was significant, $b=-1.72, p<.001$, such that one's own greater avoidant attachment was associated with one's own less functional well-being. Each of these effects remained significant 


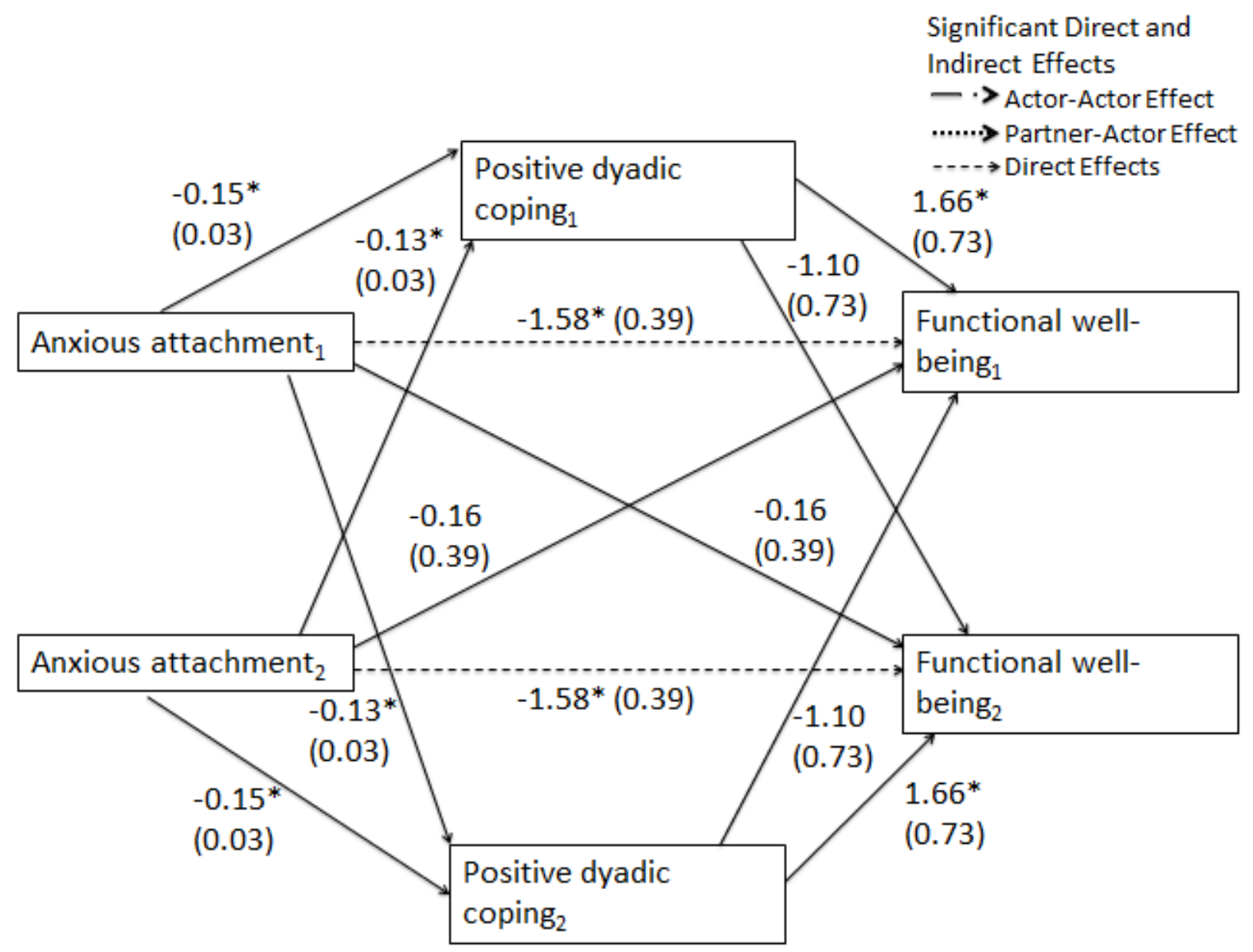

Figure 20. Positive dyadic coping as a mediator for anxious attachment and functional well-being. Note. Dashed lines indicate specific aspects of mediation such as actor-actor mediation and direct effects. Solid lines denote each pathway that was evaluated.

${ }_{1}$ and $_{2}$ refer to different members of the dyad; $* 0.05$ 


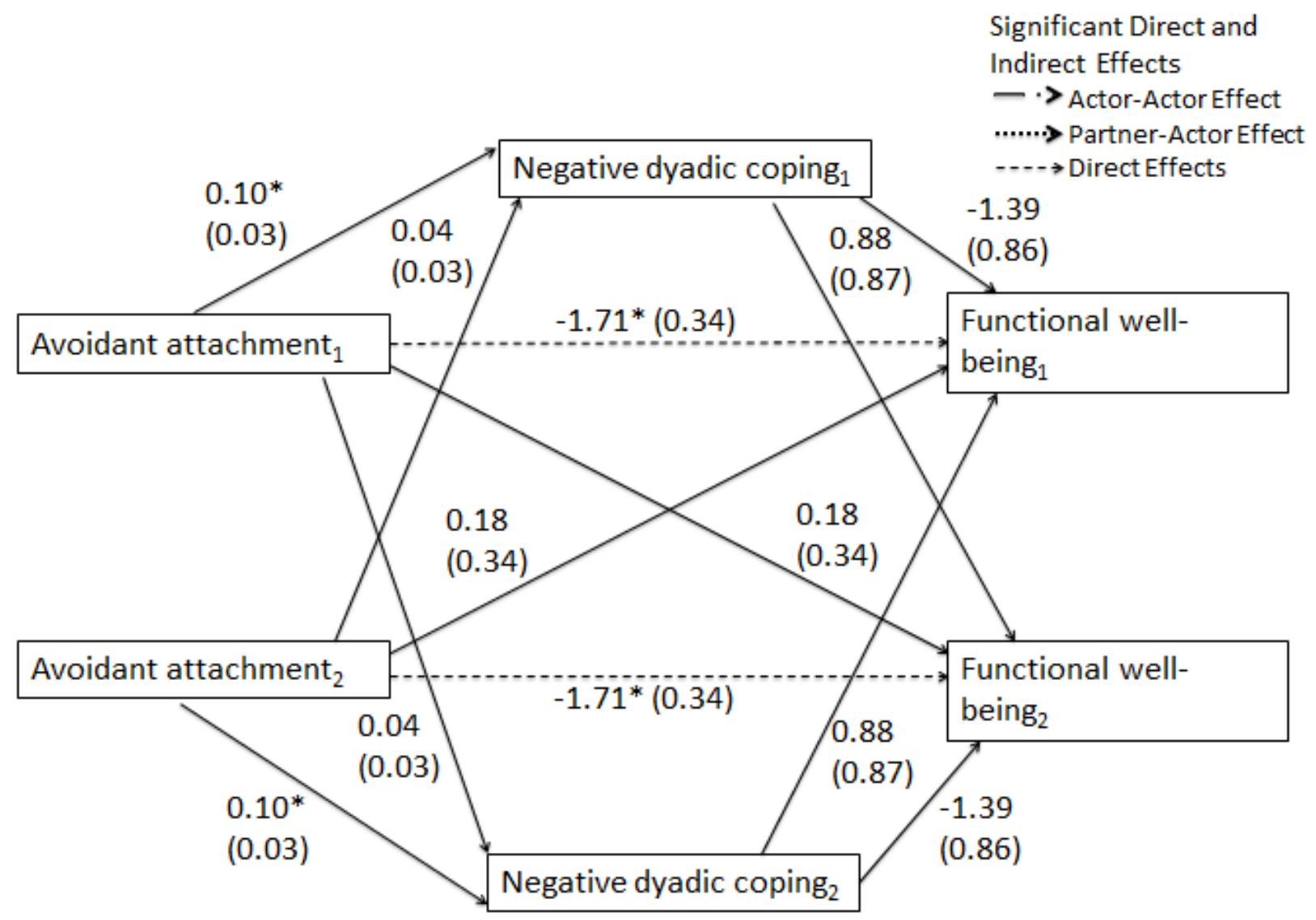

Figure 21. Negative dyadic coping as a mediator for avoidant attachment and functional well-being. Note. Dashed lines indicate specific aspects of mediation such as actor-actor medizion and direct effects. Solid lines denote each pathway that was evaluated.

${ }_{1}$ and $_{2}$ refer to different members of the dyad; $* 0<.05$ 
after applying the conservative $p$-value corrected for multiple analyses. There were no significant indirect effects.

The results of the model examining negative dyadic coping as a mediator of the effect of anxious attachment on functional well-being are displayed in Figure 22. There were significant actor, $b=0.14, p<.001$, and partner, $b=0.09, p=.002$, effects of anxious attachment on negative dyadic coping. That is, one's own and one's partner's greater anxious attachment was associated with one's own greater negative dyadic coping. The direct actor effect of anxious attachment on functional well-being was significant, $b=-1.58, p<.001$, such that one's own greater anxious attachment was associated with one's own less functional well-being. Each of these effects remained significant after applying the conservative $p$-value corrected for multiple analyses. There were no significant indirect effects.

Summary. In sum, dyadic coping did not significant mediate actor and partner effects of attachment on physical, emotional, or functional well-being. Regarding social well-being, actor positive dyadic coping significantly mediated the association between actor avoidant attachment and actor social well-being. Actor positive dyadic coping also significantly mediated the association between actor anxious attachment and actor social well-being. There was an indirect partner effect such that greater partner anxious attachment was associated with less actor positive dyadic coping, which was associated with less actor social well-being. Finally, greater actor negative dyadic coping significantly mediated the association between greater actor anxious attachment and less actor social well-being. Negative dyadic coping did not significantly mediate the association between avoidant attachment and social well-being. These models accounted for between $6-41 \%$ of the direct actor effects. 


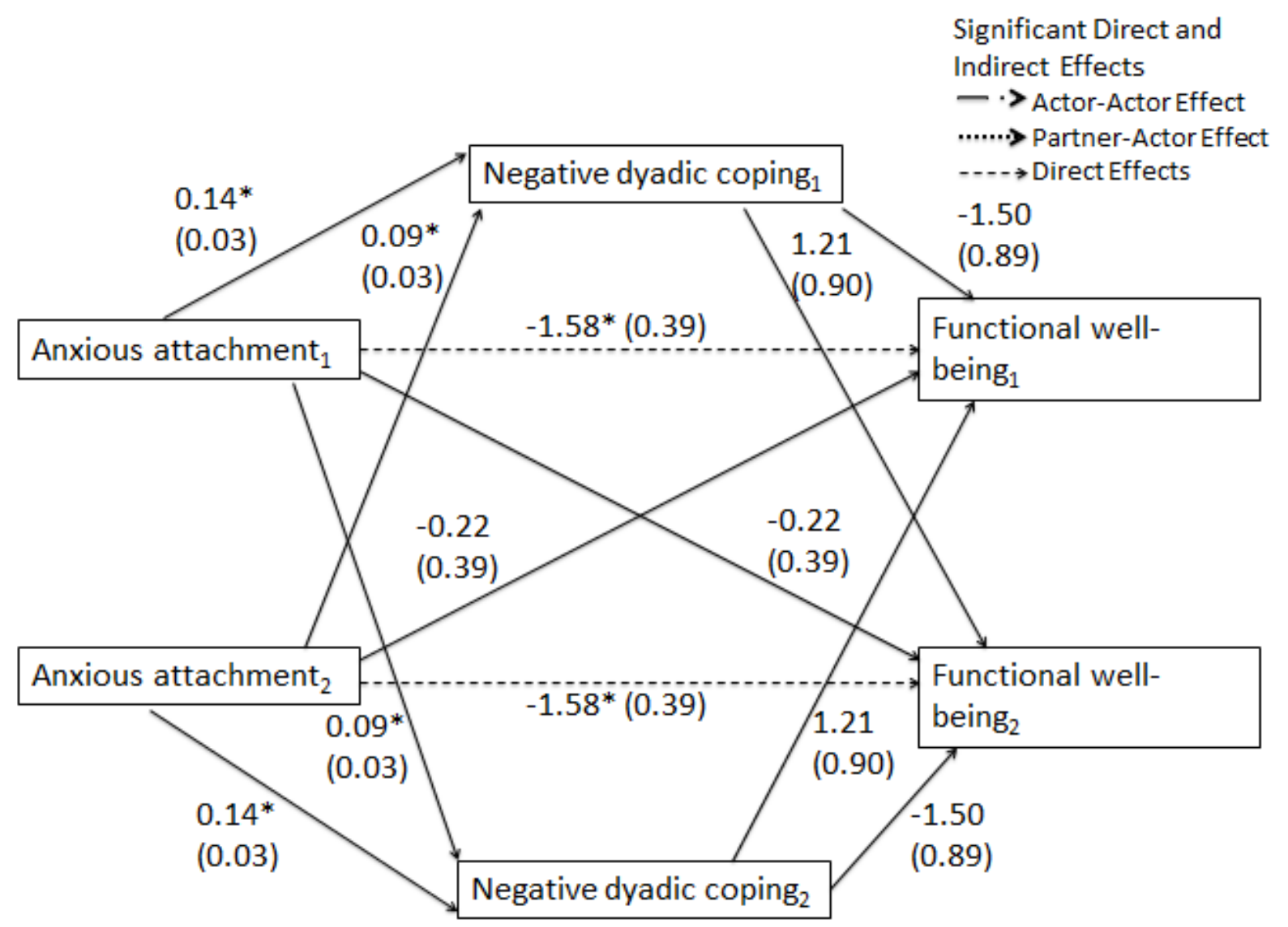

Figure 22. Negative dyadic coping as a mediator for anxious attachment and functional well-being. Note. Dashed lines indicate specific aspects of mediation such as actor-actor medizion and direct effects. Solid lines denote each pathway that was evaluated.

${ }_{1}$ and ${ }_{2}$ refer to different members of the dyad; $* 0<.05$ 


\section{Discussion}

\section{Overview of the Results}

The present study examined dyadic effects of attachment and dyadic coping on quality of life among couples facing ovarian cancer. Four quality of life outcome variables were examined: physical, social, emotional, and functional well-being. Correlations were used to examine associations between study variables for all participants. Physical well-being was significantly correlated with anxious attachment and negative dyadic coping, but not avoidant attachment or positive dyadic coping. Social well-being was significantly correlated with anxious and avoidant attachment as well as positive and negative dyadic coping. Emotional well-being was significantly correlated with anxious and avoidant attachment but not positive or negative dyadic coping. Finally, functional well-being was significantly correlated with anxious and avoidant attachment as well as positive and negative dyadic coping.

Using the APIM to account for nonindependence between dyads, there were significant actor effects of anxious and avoidant attachment for worse social, emotional, and functional well-being. There was also a significant actor effect of anxious but not avoidant attachment for worse physical well-being. There were no significant partner effects of attachment for any of the quality of life outcomes examined. Additionally, there were significant actor effects in the expected direction of positive and negative dyadic coping for social and functional well-being. Dyadic coping was not significantly associated with physical or emotional well-being. No significant partner effects were found for dyadic coping.

Mediation was assessed using the APIMeM and Sobel test. Positive and negative dyadic coping significantly mediated the associations between anxious and avoidant attachment on social well-being. There were significant actor-actor indirect effects for the mediating role of 
positive dyadic coping on avoidant attachment and social well-being; the mediating role of positive dyadic coping on anxious attachment and social well-being; and the mediating role of negative dyadic coping on anxious attachment and social well-being. That is, one's own attachment was associated with one's own dyadic coping, which was associated with one's own quality of life. There was not a significant actor-actor effect for the mediating role of negative dyadic coping on avoidant attachment and social well-being.

Interestingly, although the direct partner effect of attachment on social well-being was not significant, there were significant indirect partner effects. Specifically, the partner-actor effect was significant for positive dyadic coping and anxious attachment. That is, one's partner's greater anxious attachment was associated with one's own less positive dyadic coping, which was associated with one's own worse social well-being. Dyadic coping did not significantly mediate the effects of attachment on physical, emotional, or functional well-being.

\section{Understanding the Sample}

Medical characteristics. Within the present sample, $71 \%$ of patients were diagnosed with late-stage cancer. This is higher than the rate of $60 \%$ of incident cases diagnosed with late stage cancer generally reported (Canadian Cancer Society's Advisory Committee on Cancer Statistics, 2014). Recruitment took place at Princess Margaret Hospital, a tertiary care clinic and one of the largest cancer centres in the world. The present sample does appear comparable to that of another study in which women were recruited from specialized gynecological cancer surgery centres in Denmark (Robinson, Christensen, Ottesen, \& Krasnik, 2012). The Danish sample reported $66 \%$ of patients were diagnosed with late stage cancer, compared to $71 \%$ of the present sample, and there were identical rates of participants currently receiving treatment at the time of the study (Robinson et al., 2012). While the Danish study described $78 \%$ of their sample having 
received chemotherapy and surgery in the past, $59 \%$ of the present sample endorsed this past treatment option (Robinson et al., 2012). This may be attributable to differences in radiation therapy, while $15 \%$ of the present sample reported radiation therapy in the past, it was either not reported or not received by the Danish sample. In sum, the present sample may represent elevated late stage disease compared to population norms, but appears comparable to another sample from specialized cancer care clinics.

Attachment. The existing literature using the ECR-R does not comment on overall attachment security of samples; that is, there are no published benchmarks to determine whether the current sample reported generally high or low levels of insecure attachment. Descriptively, however, out of a possible $1-7$ range, the sample reported mean avoidant attachment of 2.48 and 2.63 for patients and spouses respectively, and mean anxious attachment of 2.13 and 2.15, demonstrating generally low levels of insecure attachment. These low levels of anxious and avoidant attachment appear to be generally comparable to that reported by other cancer samples and healthy controls. Mean avoidant attachment was 2.48 for patients in the present sample, which is slightly higher compared to 2.17 among patients with lung cancer (Porter et al., 2012) and 2.06 among healthy controls (Sibley, Fischer, \& Liu, 2005). Mean anxious attachment was 2.13 for patients in the present sample, which is slightly lower compared to 2.43 among patients with lung cancer (Porter et al., 2012) and comparable to the 2.16 among healthy controls (Sibley et al., 2005). Additionally, spouses of lung cancer patients reported mean avoidant attachment of 2.18, compared to 2.63 in the present sample, and mean anxious attachment of 2.55 , compared to 2.15 in the present sample (Porter et al., 2012); suggesting somewhat higher levels of avoidant attachment and lower levels of anxious attachment. Notably, the present sample reported more 
avoidant than anxious attachment, which is an unusual pattern (Chopik, Edelstein, \& Fraley, 2013; Porter et al., 2012; Sibley et al., 2005).

Age-related patterns of attachment may help explain these differences. A cross-sectional study of age-related differences in attachment revealed attachment anxiety appeared to be lower among middle- and older adults (Chopik et al., 2013). The mean age of the current sample is approximately 60 years old, representing a generally older sample. A brief re-examination of the means of anxious and avoidant attachment separated by age revealed that among participants aged 60 and older, mean avoidant attachment was 2.53 and mean anxious attachment was 1.99; participants aged 59 and younger reported a comparable mean avoidant attachment of 2.59 and a greater mean anxious attachment of 2.32. Although avoidant attachment was still higher than anxious attachment at each age range, this pattern is consistent with the suggestion that there were reduced levels of anxious attachment in the older age group.

Dyadic coping. The present sample reported generally high levels of positive dyadic coping and low levels of negative dyadic coping. There was greater use of positive than negative dyadic coping. These findings also appear to be comparable to that reported by other healthy populations (Vaske et al., 2015). For example, among patients with chronic obstructive pulmonary disease, mean total dyadic coping was 133.32 (Vaske et al., 2015), this appears to be comparable to the mean total dyadic coping (higher scores indicate more positive dyadic coping) of patients in the present sample, which was 136.80. Additionally, spouses of chronic obstructive pulmonary disease patients reported a mean dyadic coping total score of 126.66 (Vaske et al., 2015), which is similar but slightly lower than the 133.83 mean dyadic coping total score reported by spouses in the present sample. This difference appears to be relatively small considering the possible range of 37 to 185 on the total dyadic coping score. These results 
additionally appear comparable to the German, Italian, and French validation samples of the DCI, which reported mean total dyadic coping scores of $133.50,125.57$, and 126.00 , respectively (Ledermann et al., 2010). Total scores were used to compare across studies, as there is currently no published literature on the means of positive dyadic coping and negative dyadic coping total scores.

Quality of life. As previously stated, patients in the present study endorsed significantly less functional well-being compared to the validation sample of ovarian cancer patients (BasenEngquist et al., 2001). This is surprising as patients in the present study were comparable in the amount of participants with advanced stage illness, number of patients on active treatment, and the frequency of relapse (Basen-Engquist et al., 2001). As Princess Margaret Hospital is a research and teaching institute many of the participants were likely involved with clinical trials, although unfortunately this information was not collected. It is possible that the treatment that they were receiving had side effects that were not as well-known and were more intrusive. Additionally, functional well-being in the present study was comparable to that reported by patients in the control arms of two randomized trials of ovarian cancer treatment (von Gruenigen et al., 2010). Moreover, the study from the validation sample took place in the United States, whereas the present study took place in Canada, reflecting vastly different healthcare systems. The difference in functional well-being may reflect a selection bias of the hospital itself if, as a result of the universal healthcare system in Canada, individuals with less functional well-being had greater access to treatment than individuals in the United States.

As was also previously stated, spouses in the present sample endorsed significantly greater physical and functional well-being than the general population (Brucker et al., 2005). This finding is inconsistent with the limited extant literature; however, the few studies 
demonstrating impaired quality of life among spouses of cancer patients have been limited as they were descriptive in nature (Arden-Close et al., 2013). For example, in a study of couples facing ovarian cancer, spouses appeared to endorse a lower rating of quality of life compared to both healthy controls and the patients themselves, however it was not investigated whether these differences were statistically significant, and they only examined total quality of life as opposed to the subscales (Arden-Close et al., 2013). It may be that spouses of cancer patients in the current study make comparisons to their ill partners when they are evaluating their health, thus casting a more favourable view of their own health and capabilities. A study that evaluated the degree to which social comparisons affect older adults' quality of life, supported the effects of downward social comparisons, such as "Some people have it much worse [...] I'm pretty healthy," on perceived health and well-being (Chandler, 2010).

Finally, spouses reported significantly less social well-being than patients. This is consistent with a study that found that ovarian cancer patients reported better social well-being compared to normative data (von Gruenigen et al., 2010). The authors suggested that this finding reflects that patients with cancer receive greater social support (von Gruenigen et al., 2010). Similarly, social well-being of patients in the present study may have benefited from increased social support, whereas spouses may be overlooked in favour of supporting the patient. This is supported by a qualitative study in which the wife of a cancer patient stated, "You don't feel you can be saying 'what about me?' you sort of think 'no you shut up, you are not the one with the problem [...]' you don't feel you have a right to ask for help" (Foster et al., 2015).

\section{Dyadic Effects of Attachment on Quality of Life}

Actor effects. The present study demonstrated significant actor effects of anxious and avoidant attachment with each facet of quality of life, with the exception of avoidant attachment 
and physical well-being. Greater actor anxious attachment was associated with worse actor physical, social, functional, and emotional well-being. Greater actor avoidant attachment was associated with worse actor social, functional, and emotional well-being. Furthermore, these associations were true for both patients and spouses; analyses demonstrated that the strength of the associations between attachment and quality of life did not differ in magnitude for patients versus spouses.

These findings suggest that the internal working models that an individual holds about their own self-worth and self-value in relationships, as well as the responsiveness and dependability of close others, significantly influences their perceptions of their own health. These results are consistent with the model proposed by Pietromonaco and colleagues (2013) as well as previous research (Hsieh et al., 2014; Porter et al., 2012). Greater insecure attachment has been shown to result in less constructive affective and cognitive responses to stress (Shaver \& Mikulincer, 2008), greater physiological and subjective reactivity (Maunder, Lancee, Nolan, Hunter, \& Tannenbaum, 2006), and less flexible and effective coping strategies (Schmidt et al., 2002). These hyperactivating and deactivating responses ultimately intensify distress and increase likelihood of difficulty adjusting (Shaver \& Mikulincer, 2008).

The current study adds to the literature by examining health outcomes, including both members of the dyads, and making use of sophisticated statistical methods to examine dyad-level associations. Most of the research on attachment and ovarian cancer has examined outcomes such as anxiety, depression, and social support (Nissen, 2016). The present findings further the literature that suggests that relationship variables affect not only relational and mental health outcomes, but also physical health concerns. Only one prior study examined attachment and quality of life among the ovarian cancer population, despite the unique health presentation of this 
population, and it did not include spouses (Hsieh et al., 2014). Additionally, prior studies of attachment and quality of life within the cancer population that have included both patients and spouses have not used dyadic data analyses to account for nonindependence (Porter et al., 2012).

Physical well-being. There were significant actor effects of insecure attachment for each of the categories of quality of life with the exception of avoidant attachment on physical wellbeing. This is consistent with previous studies that have found significant associations between attachment and psychological or social aspects of well-being but not physical health (Ávila et al., 2015; Porter et al., 2012). Additionally, a previous study of gynaecological cancer patients similarly found that after controlling for sociodemographic and general health variables, anxious and not avoidant attachment was significantly associated with physical quality of life (Hsieh et al., 2014).

This finding is consistent with the deactivating strategies that are characteristic of avoidant and not anxious attachment, which include downplaying or suppressing threat-related thoughts and cues (Shaver \& Mikulincer, 2011). Indeed, a study of the somatization of physiological complaints found anxious and not avoidant attachment to be associated with greater self-reported symptom levels (Schmidt et al., 2002). Moreover, whereas anxious attachment has been associated with subjective levels of reactivity to a stressor, avoidant attachment was associated with objective, specifically heart rate variability, and not subjective measures (Maunder et al., 2006).

Partner effects. There were no partner effects of attachment on any of the quality of life indices. The results of the present study suggest that the attachment orientation of one's partner does not have a significant effect on one's own quality of life. This was unexpected, however, the previous literature has been limited. There have not been many studies that directly 
examine the partner effect of attachment on quality of life. In one of the few studies to examine this association, Porter and colleagues (2012) found a single partner effect, such that spouse avoidant attachment was significantly associated with patient functional well-being. Spouse anxious attachment was not associated with patient physical, functional, or social well-being, and spouse avoidant attachment was not associated with patient physical or social well-being (Porter et al., 2012). The emotional well-being subscale was not examined, nor was the effect of patient attachment on spouse quality of life (Porter et al., 2012). Moreover, this study was completed with lung cancer patients and the results were limited by their use of multiple regression analyses as opposed to analyses designed for dyadic data. The additional extant literature of couples facing cancer has found the presence of cross-dyadic associations of other variables, such as distress and illness beliefs, predicting quality of life (Kim et al., 2008; Wu et al., 2013) or crossdyadic associations of attachment predicting other variables, such as marital satisfaction, caregiving, and cortisol (Braun et al., 2012; Hsiao et al., 2014; Shields, Travis, \& Rousseau, 2000).

The lack of partner effects may reflect the findings that one's own attachment influences the perception of received support. In a seminal paper on attachment and perceptions of social support, Collins and Feeney (2004) found that individuals who were insecurely attached rated their partners as less supportive, even after controlling for observer's ratings of support. That is, individuals who were higher in anxious and avoidant attachment found their partners to be less supportive, regardless of the amount of observer-rated support provided. Thus, it may be that partner attachment was not significantly associated with one's own well-being, because one's own insecure attachment negatively filters the way supportive partner behaviours are perceived 
(Collins \& Feeney, 2004). Therefore, one's own insecure attachment strongly influences the perception of support, despite what one's partner is providing according to observers.

Not only does one's own attachment bias the interpretation of partners' behaviours and responsiveness, a recent study suggests that there may be a disconnect between an individual's attachment orientation and one's own behaviours. A study of conflict amongst newlyweds found a significant interaction between an individual's avoidant attachment and observer-rated responsiveness in predicting that person's own perceived responsiveness (Beck, Pietromonaco, DeVito, Powers, \& Boyle, 2014). The more an observer rated an avoidantly attached individual as being responsive to his or her partner, the less that avoidantly attached individual perceived themself as being responsive. The authors suggested that individuals reconciled their supportive behaviours with their fear of intimacy by believing they were being unresponsive despite what was objectively occurring (Beck et al., 2014). Although majority of the literature suggests that insecure attachment is in fact associated with unhelpful behaviours such as distancing and avoidance (Shaver \& Mikulincer, 2011), perhaps there are circumstances in which individuals with avoidant attachment in fact engage in supportive behaviours, regardless of their internal working model, which may have reduced the negative effects of avoidant attachment on partner quality of life in the present study.

The present sample also represents an older group of individuals, with most couples having been in their partnerships for a long time. As was previously discussed, the nature of attachment has been shown to change over time (Kafetsios \& Sideridis, 2006). Studies have found that there are lower rates of anxious attachment itself in middle aged and older adults, and this is especially true for individuals who are partnered (Chopik et al., 2013). Additionally, the link between insecure attachment, anxious attachment in particular, and well-being has been 
shown to be weaker in older populations (Kafetsios \& Sideridis, 2006; Li \& Fung, 2014). It has been suggested that the individuals learn with time to manage the cognitive and emotional dysregulation associated with insecure attachment. Although the changes in the strength of partner effects of attachment on well-being over time have not been examined, it is possible that the strength of these associations also diminish over time as individuals have more practice coping with stressors as a couple.

\section{Dyadic Effects of Dyadic Coping on Quality of Life}

Actor effects. There were significant actor effects of positive and negative dyadic coping on social and functional well-being. This is consistent with the model by Pietromonaco and colleagues (2013), which suggests that dyadic processes are associated with health outcomes, as well as the existing literature (Feldman \& Broussard, 2006; Hill, 2016; Regan et al., 2014; Rottmann et al., 2015; Song et al., 2011; Vaske et al., 2015). Furthermore, these associations were supported for both patients and spouses; analyses demonstrated that the strength of the associations between dyadic coping and quality of life did not differ in magnitude for patients versus spouses. These results suggest that the more individuals perceive themselves and their partner are working together to jointly face this stressor - by supporting one another, sharing the burden of responsibilities, jointly problem solving, and refraining from hostility or blaming - the better their own adjustment.

This study makes a meaningful contribution to the literature as it is among the first to expand Bodenmann's systemic dyadic coping conceptualization beyond its effects on relationship outcomes (Bodenmann, 2005). In addition, it is the first to examine Bodenmann's systemic dyadic conceptualization of coping and quality of life within a cancer population, with only two prior studies of dyadic coping and quality of life, among COPD patients (Vaske et al., 
2015) and dementia patients and their caregivers (Häusler et al., 2016). Previous quality of life studies with cancer patients and their partners have examined open communication (Song et al., 2011) and individual coping (Kershaw et al., 2008), as well as received social support (Hill, 2016), but not dyadic coping. For example, Kerhsaw and colleagues (2008) found that greater active and less avoidant coping was associated with better patient mental health quality of life and less avoidant coping was associated with better spouse mental health quality of life among couples facing prostate cancer. Although coping was examined in both members of the dyad, it was still conceptualized as an individual level process. The systemic conceptualization of dyadic coping by Bodenmann (2005) has been shown to predict interpersonal and health outcomes beyond that of individual coping (Bodenmann et al., 2011).

The results of the present study are consistent with prior research that has examined associations between dyadic coping and anxiety and depression in cancer patients and spouses (Regan et al., 2014; Rottmann et al., 2015). Building upon the model by Pietromonaco and colleagues (2013), the present study uniquely expands the literature by demonstrating that this relationship process has significant associations with health outcomes, especially health-related quality of life. Furthermore, this study extends the framework proposed by Pietromonaco and colleagues (2013). The authors suggest a number of dyadic process that may be associated with health outcomes, including support-seeking, caregiving, social negativity, relationship satisfaction, and commitment. The authors specifically suggest that this is a general guideline that may be expanded to include additional dyadic processes (Pietromonaco et al., 2013); indeed, the present findings suggest that dyadic coping is associated with health outcomes, and sets the stage to examine whether it is a pertinent mediator between attachment and quality of life.

\section{Domains of quality of life.}


The effects of dyadic coping differed by the domains of quality of life. Positive and negative dyadic coping were significant predictors of social and functional well-being, and not physical and emotional well-being. A better understanding of these results may benefit from a closer examination of the items within each of these categories. Questionnaire items of social well-being reflect a closeness to others, feeling supported, and satisfaction with communication, such as, "I get emotional support from my family." These are all areas that would be expected to be associated with a construct that is assessing the degree to which couples work together to address stressors. Functional well-being was also significantly associated with dyadic coping. Items on this scale focus on enjoyment of life and illness acceptance as opposed to activities of daily living. For example, items include "I am able to enjoy life" and "I have accepted my illness". The significant effects of dyadic coping on this outcome may be reflecting the utility of partner support in reframing stressful events. For example, one of the items of positive dyadic coping includes, "My partner helps me to see stressful situations in a different light."

Physical well-being, on the other hand, measures physical symptoms such as pain, nausea, and fatigue. That dyadic coping was not found to be associated with physical well-being is consistent with prior studies showing individual coping to be more reliably associated with mental and not physical quality of life within the cancer population (Boehmer, Luszczynska, \& Schwarzer, 2007; Kershaw et al., 2008; Kershaw, Northouse, Kritpracha, Schafenacker, \& Mood, 2004; Lafaye et al., 2014). Additionally, a study examining the effect of dyadic coping within a healthy population found that dyadic coping was less powerful in explaining variance in well-being, especially physical symptoms, anxiety, social dysfunction, and depression, than relationship outcomes (Bodenmann et al., 2011). It may be that the presence and severity of 
physical symptoms associated with cancer and treatment side effects cannot be as effectively addressed with individual and dyadic coping strategies.

It is surprising that emotional well-being was not significantly associated with dyadic coping. This subscale assesses sadness and worry, for example, "I feel sad" and "I feel nervous." As this construct represents internal emotional processes, perhaps it is more strongly associated with individual as opposed to dyadic coping. There is partial support for this hypothesis in the literature. A study of dyadic coping among male spouses of breast cancer patients similarly failed to find significant effects of positive or negative dyadic coping on emotional well-being (Feldman \& Broussard, 2006). Meanwhile, individual coping has been shown to be significantly associated with emotional well-being (Boehmer et al., 2007; Kim, Han, Shaw, McTavish, \& Gustafson, 2010). For example, a study that examined individual coping strategies on emotional, social, and physical well-being after tumor surgery found significant effects on emotional wellbeing; specifically, active coping was significantly associated with greater emotional well-being (Boehmer et al., 2007).

Partner effects. There were no partner effects of dyadic coping on any of the quality of life indices. The results of the present study suggest that the extent to which one's partner perceives that he or she is engaging in more dyadic coping does not have a significant effect on one's own quality of life. This unexpected finding is inconsistent with the limited extant literature. For example, a study of couples in which one partner was diagnosed with chronic obstructive pulmonary disease (COPD) found a few significant partner effects of dyadic coping on total quality of life (Vaske et al., 2015). For example, APIM analyses demonstrated that patients reported better quality of life when their spouse reported that they and their partner communicate more about their stress, and spouses reported better quality of life when the patient 
described more use of delegated coping (Vaske et al., 2015). The main symptom of COPD is shortness of breath, which makes, for example, carrying heavy items and going up and down stairs difficult. It may be that there is more that partners can do to offset the symptoms, and improve quality of life, of individuals with COPD than cancer symptoms.

The lack of partner effects is also at odds with a previous study of "we talk" among couples facing head and neck cancer (Badr et al., 2016). Patients and spouses reported less psychological distress when their partner used more "we talk" and reported less positive affect when their partner used more "I talk" in conversations about cancer (Badr et al., 2016). There are important differences between the present study and that by Badr and colleagues (2016), which may explain the discrepancy in findings. "We talk," as measured in the study by Badr and colleagues (2016), and dyadic coping, as measured by the DCI, differ as "we talk" appears to measure appraisal of cancer as a dyadic stressor as opposed to the DCI, which is more behavioural in nature. Perhaps dyadic appraisal is more important for emotional outcomes than dyadic behaviour.

There is some additional support for this hypothesis from a study of couples facing chronic heart failure (Rohrbaugh, Mehl, Shoham, Reilly, \& Ewy, 2008). Greater "we talk" by the spouse predicted improved patient heart failure symptoms over time (Rohrbaugh et al., 2008). A self-report measure, which included both self-reported dyadic appraisal, "To what extent do you [view problems related to the heart condition] as 'our problem,"' and self-reported dyadic behavior, "When a problem related to your heart condition arises, to what extent do you and your partner work together to solve it," was not significant (Rohrbaugh et al., 2008). It is possible, that the communication of appraisal of significant stressors as shared stressors has a more significant effect than the coping behaviours themselves. The study by Rohrbaugh and colleagues (2008) 
also suggests that observational measurement of dyadic coping may more effectively capture important relationship dynamics than self-report.

\section{Dyadic Coping as a Mediator Between Attachment and Quality of Life}

Actor-actor indirect effects for social well-being. Actor positive and actor negative dyadic coping were significant mediators of the association between actor anxious attachment and actor social well-being. An individual's own greater anxious attachment was associated with one's own less positive and more negative dyadic coping, which was associated with one's own lower social well-being. Actor positive dyadic coping was also a significant mediator of the association between actor avoidant attachment and actor social well-being. An individual's own greater avoidant attachment was associated with one's own less positive dyadic coping, which was associated with one's own lower social well-being. These results suggest that the effect of individuals holding unhelpful internal working models about the reliability of others and about their own self value on social quality of life was, in part, accounted for by engaging in less shared positive and more joint negative dyadic coping.

These results are consistent with the model by Pietromonaco and colleagues (2013), in which they suggest that attachment negatively impacts health outcomes by way of dyadic processes. Furthermore, these results make a significant contribution to the literature, as it is one of the first studies examining dyadic coping as a mechanism for the negative effect of attachment. Previous studies have found dyadic coping to be a mediator of negative health outcomes (Häusler et al., 2016; Johnson et al., 2013). For example, among dementia patients and their spousal caregivers, dyadic coping significantly mediated the association between perceived stress and quality of life for spouses (Häusler et al., 2016). However, few studies have examined it as a mediator of the negative effects of attachment (Kardatzke, 2010; Levey, 2003), and none 
with a health outcome. A dissertation study found that total dyadic coping significantly mediated the association between anxious attachment and marital satisfaction, as well as the association between avoidant attachment and marital satisfaction, among graduate students (Kardatzke, 2010).

Additionally, the results of the present study extend the literature showing individual coping to mediate the associations between attachment and negative health outcomes (Aarts, Hinnen, Gerdes, Acherman, \& Brandjes, 2014; Wei, Heppner, \& Mallinckrodt, 2003). For example, individual coping styles were found to mediate the associations between anxious attachment and physical well-being, anxious attachment and mental well-being, and avoidant attachment and mental well-being among bariatric surgery patients (Aarts et al., 2014). The present study not only furthers this research by examining dyadic coping as opposed to individual coping, it uniquely includes both the patient and their spouse, acknowledging both members of the dyad facing this common stressor.

Partner-actor indirect effect for social well-being. Although there were no significant direct partner effects of attachment on quality of life, there was a significant indirect partner effect of anxious attachment on social well-being by way of actor positive dyadic coping. An individual's partner's greater anxious attachment was associated with one's own less positive dyadic coping, which was associated with their own impaired social well-being. These results suggest that the more one partner holds an insecure internal working model, the less the other partner engages in joint positive coping, which results in lower social quality of life. This finding highlights the unique contributions of examining couples using a dyadic framework, allowing for both intrapersonal and interpersonal paths of mediation. 
These results suggest there are important interpersonal effects of attachment on dyadic processes. This is consistent with the literature that has associated insecure attachment of one partner with dyadic processes and outcomes of the other partner (Braun et al., 2012; Givertz, Woszidlo, Segrin, \& Knutson, 2013; Millings \& Walsh, 2009; Shields et al., 2000). Anxious attachment, and likely the resulting unhelpful hyperactivating behaviours and cognitions such as catastrophizing and excessive reassurance seeking, elicits a less positive coping response from their partner. For example, among older couples with cancer, greater insecure attachment reported by wives was associated with worse marital satisfaction among their husbands (Shields et al., 2000). Additionally, one's partner's greater insecure attachment was significantly associated with one's own less caregiving sensitivity among long-term couples (Millings \& Walsh, 2009).

Mediation of functional well-being. Although both insecure attachment and dyadic coping were significantly associated with functional well-being, dyadic coping did not significantly mediate this association. That is, the association between unhelpful internal working models and functional well-being was not attributable to dyadic coping strategies. This is inconsistent with the previously cited literature in which individual coping mediates the association between attachment and quality of life (Aarts et al., 2014; Wei et al., 2003), and a single dissertation in which dyadic coping was a significant mediator between attachment and relationship outcomes (Kardatzke, 2010). However, it is consistent with existing research that has found that social support, which is not the same as dyadic coping but is a component of it, does not mediate the association between attachment and negative outcomes (Monin, Zhou, \& Kershaw, 2014; Robles, Brooks, Kane, \& Schetter, 2013). A study of couples coping with pain found that support did not significantly mediate the associations between attachment and 
depression and relationship satisfaction (Monin et al., 2014). Additionally, supportiveness, as well as stress appraisals, emotions, and cortisol changes, did not significantly mediate the association between attachment and skin barrier recovery, a physiological measure of response to stress, after a stressful discussion among dating couples (Robles et al., 2013).

It may be that alternative mechanisms are more important for the other domains of quality of life. For example, emotion regulation is integrally related to attachment, and has consistently been demonstrated to be a significant mediator between insecure attachment, particularly anxious attachment, and depression (Malik, Wells, \& Wittkowski, 2015). Among breast cancer patients, emotion regulation strategies of communicating emotions, emotional control, and rumination, significantly mediated the association between attachment and physical, psychological, and social adjustment (Ávila et al., 2015). Self-efficacy or confidence in one's ability to cope may also be a relevant construct. To the extent that an individual has had a secure base in which to feel loved, valued, and had a sense of self-confidence instilled in them, they may have a greater sense of confidence in their ability to properly adjust and effectively cope when bad things happen, leading to better acceptance and better ability to find enjoyment and meaning in objectively challenging situations. A study of attachment in university students measured what the authors referred to as preventative coping resources, described as beliefs that one can cope successfully with life's demands, such as "I can handle most things" and "I am able to avoid causing myself stress by keeping things in perspective" (McCarthy, Lambert, \& Moller, 2006). This confidence in one's ability to cope as well as emotion regulation, significantly mediated the association between attachment and stress (McCarthy et al., 2006). Within the health psychology literature, greater insecure attachment has been shown to be significantly 
associated with reduced pain self-efficacy among chronic pain patients (Meredith, Strong, \& Feeney, 2006).

\section{Clinical Implications}

Implications for healthcare professionals. The results of the present study suggest that attachment and dyadic coping are important considerations for health and psychological interventions for couples coping with ovarian cancer. Healthcare providers may benefit from a greater understanding of patterns of attachment and the effects that hyperactivating and deactivating strategies have on illness behaviour (Hunter \& Maunder, 2001). The results of the present study provide healthcare professionals with a framework for understanding the individual differences in physical, social, functional, and emotional well-being that they observe within their patient population as well as their spouses. For example, healthcare professionals may consider providing more explicit instructions of what partners can do to help one another when they notice couples engaging in deactivating coping strategies, with the understanding that intimacy and responsiveness is not something that these couples may intuitively engage in. Additionally, an awareness of the excessive reassurance seeking that is characteristic of anxious attachment can help healthcare professionals address the unhelpful effects of excessive reassurance seeking on well-being, and discuss alternative ways of asking for support and clarification, in a way that is direct and empathic.

These present findings also highlight the importance of healthcare providers conceptualizing and framing the cancer experience as a shared stressor between both members of the couple. They may consider making deliberate efforts to include spouses in the cancer experience and validating the experience as a stressor at the level of the couple, as opposed to viewing the couple as a patient and a support person. Healthcare professionals should consider 
relational concerns and interpersonal communication within the couple as an integral aspect of the treatment of ovarian cancer patients that they can inquire about and offer to provide services or referrals if necessary to help couples find support. Couples-based interventions for cancer have demonstrated efficacy (Badr \& Krebs, 2013; Manne, Siegel, Heckman, \& Kashy, 2016; Scott, Halford, \& Ward, 2004). For example, in a study of breast and gynecological cancer patients, a couples-based intervention significantly improved couples' communication and coping, as well as patients' distress and avoidance (Scott et al., 2004). Additionally, prostate cancer patients who received a couples-based intervention compared to care as usual reported significantly improved quality of life (Campbell et al., 2007).

Clinical implications of the role of attachment. There were significant intrapersonal and interpersonal effects of attachment on well-being. Clinically, attachment can be considered both a treatment target as well as a feature of case conceptualization. Although attachment is generally considered stable, there is evidence that psychological treatment can alter these internal working models (Moser et al., 2016; Taylor, Rietzschel, Danquah, \& Berry, 2015). A review of 14 studies revealed significant increases in attachment security after therapy across a variety of therapeutic orientations including psychodynamic, interpersonal, cognitive behavioural, attachment-focused, and person-centered therapies (Taylor et al., 2015). For example, a study of integrative behavioural couples therapy in distressed couples examined changes in attachment and changes in relationship satisfaction over time (Benson, Sevier, \& Christensen, 2013). Lagged prediction models suggested that marital satisfaction changed first, which then lead to changes in attachment security (Benson et al., 2013). Attachment research is increasingly focused on the ability to shift internal working models through deliberate therapeutic efforts and as secondary gains from general improvements in dyadic processes. However, none of these studies have 
examined changes in attachment after therapy among cancer or any other medical populations (Taylor et al., 2015).

A review of couple processes within breast cancer suggested that Emotion Focused Therapy (EFT) should be considered as a treatment option for couples facing cancer (Naaman, Radwan, \& Johnson, 2009). EFT is rooted in attachment theory, with a goal to foster greater security between spouses and improve dyadic interactions (Naaman et al., 2009). These positive changes will allow for greater strength and resiliency within the dyad to face adverse events such as those associated with the cancer trajectory (Naaman et al., 2009). The usefulness of EFT among couples facing cancer has yet to be examined.

Although attachment is often associated with the psychodynamic and emotion focused theoretical orientations, there are significant applications of attachment within cognitive behavioural therapy (CBT), especially for case conceptualization (McBride \& Atkinson, 2009). In particular, the conceptualization of attachment as mental representations that guides the content of beliefs, attitude, and memories, complements Beck's cognitive theory, which states that people create cognitive structures or cognitive representations of their experience called schemas (McBride \& Atkinson, 2009; Platts, Tyson, \& Mason, 2002). The specific content of schemas are referred to as core beliefs, which guide intermediate beliefs - that of underlying assumptions, rules, and attitudes - and ultimately affect everyday automatic thoughts in response to situations (Beck, 2011). Core beliefs essentially fall into the main categories of helplessness, unlovability, and worthlessness and can be about the self as well as other people, such as 'other people are untrustworthy' (Beck, 2011). This reflects similarities to the attachment conceptualization of self - seeing oneself as loveable and worthwhile - and other - seeing others as reliable and trustworthy - described by Bartholomew and Horowoitz (1991). 
Knowing a clients' attachment, and the effects of attachment on quality of life, will help clinicians formulate and hypothesize likely core beliefs and their consequences (McBride \& Atkinson, 2009). For example, a cancer patient who is more anxiously attached may be anticipated to hold the core belief "I am unlovable," (McBride \& Atkinson, 2009) and associated underlying assumptions such as, "if I make things too difficult, my partner will leave me" resulting in behaviours such as being overly agreeable, at the expense of their own quality of life. A study of core belief content among patients completing online CBT revealed that attachmentrelated core beliefs (such as core beliefs related to attachment figures, expressing attachment associated fears such as fear of abandonment, and unlovability) were among the most commonly identified core beliefs (Millings \& Carnelley, 2015). Once these underlying organizing structures are identified, they can be challenged and modified through cognitive restructuring, such as positive data logs and extreme contrasts, and behavioural interventions such as behavioural experiments (Beck, 2011).

Knowledge of the effects of attachment may also help clinicians anticipate and understand obstacles in psychotherapy (Platts et al., 2002). For example, if someone is high in avoidant attachment, the therapeutic alliance is going to take longer and be more difficult to establish (Platts et al., 2002). Someone who is more anxiously attached may be more likely to contact their therapist between sessions; by understanding that within the attachment framework, this behaviour is an excessive means to seek reassurance that their therapist is dependable and cares about them, the clinician can then respond appropriately (Platts et al., 2002).

Clinical implications of the role of dyadic coping. One of the ways in which attachment negatively impacts well-being is through dyadic coping; specifically, dyadic coping significantly mediated the associations between attachment and social well-being. These results 
suggest that interventions to address dyadic coping may have significant intrapersonal and interpersonal effects on social well-being. As such, Couples Coping Enhancement Training, developed by Bodenmann and colleagues based upon the dyadic coping model, which emphasizes the improvement of individual and dyadic coping skills, may be useful for ovarian cancer patients and their partners (Bodenmann \& Shantinath, 2004). Studies have demonstrated the significant efficacy of this intervention on improving marital satisfaction and dyadic coping among distressed couples (Bodenmann, Charvoz, Cina, \& Widmer, 2001). However, Couples Coping Enhancement Training has not been studied with medical populations, nor has its effectiveness for health-related outcomes been examined.

There is evidence of efficacy of an intervention study to enhance communal coping, appraising the chronic health stressor as "our" problem rather than "yours" or "'mine," on health outcomes (Rohrbaugh, Shoham, Skoyen, Jensen, \& Mehl, 2012). Health-compromised smokers and their partners completed a smoking cessation intervention program designed to enhance communal coping. Increases in "we" talk by both partners throughout the therapy sessions predicted more successful smoking abstinence one year later (Rohrbaugh et al., 2012). Intervention studies designed to enhance dyadic coping and communal coping may be useful in improving dyadic processes and quality of life, particularly social well-being, among couples coping with ovarian cancer.

\section{Limitations}

The present study is limited by a low response rate of $32.2 \%$, which may limit generalizability to the ovarian cancer population. The present sample may represent healthier patients, more stable and satisfied couples, and more highly educated participants than the general population (Reisine, Fifield, \& Winkelman, 2000). However, response rates are known 
to be lower in couples' research (Dagan \& Hagedoorn, 2014). For example, in a study of distress among couples facing breast cancer, the couples' response rate was 38\% (Hinnen et al., 2008). In couples' research, both members of the dyad must participate, if one is not interested or wants to withdraw, then both are lost (Dagan \& Hagedoorn, 2014). Additionally, recruitment is made more difficult by indirect recruitment; that is, as per most Research Ethics Boards requirements, the patient must invite their spouse to participate in the study, the researchers are not able to directly approach spouses (Dagan \& Hagedoorn, 2014). This was the case with the current study, and recruitment of the couple in the current study was a multi-step process if the spouse was not present in clinic, allowing for many stages in which participants may be lost. A systematic review of response rates in observational research studies of couples coping with cancer reported a wide range of couples' response rates from $25 \%$ to $90 \%$, with a mean of $58 \%$ and standard deviation of 17\% (Dagan \& Hagedoorn, 2014).

Moreover, Dagan and Hagedoorn (2014) raised a significant challenge in couples’ research, which is that depending on recruitment methodology, a true couples' response rate can sometimes not be calculated. The couples' response rate is the number of couples included in the final analyses divided by the initial eligible partnered patients/couples that were approached (Dagan \& Hagedoorn, 2014). If an unpartnered patients approach is used, that is, if researchers ask single or partnered patients to participate, with their partner if applicable, then there will be a subset of the sample who declined at the outset, for whom their relationship status is not known, and therefore it is not known whether they should be included as eligible, approached participants. As the present study was being conducted concurrently with a longitudinal study of ovarian cancer patients, this unpartnered patients approach was used. To be conservative, patients who were medically eligible but declined before the researchers were able to ascertain 
their relationship status were included in the calculations as eligible patients. Thus, it is important to note that although the low response rate of the current study is a significant limitation, it is also inflated by the inclusion of patients who may or may not have been eligible on the basis of their relationship status.

Another limitation is that information about whether participants were involved in clinical treatment trials, and what their trial involved, was not collected. As Princess Margaret Hospital is a research and training institute, a substantial minority of participants were involved in clinical trials. Having this information could have helped provide a context for physical symptoms and impairment experienced by the participants in the present sample, and may have enriched the clinical picture of the present sample and how it compares to other samples within the literature. It also would have been useful to understand whether elements of quality of life and the effects of attachment and dyadic coping on quality of life differed for participants in clinical trials compared to usual treatment. For example, a trial examining the safety and efficacy of adding intravenous bevacizumab to standard intravenous chemotherapy compared quality of life between bevacizumab and chemotherapy as usual groups (Stark et al., 2013). Bevacizumab was associated with small but significant decrements in quality of life compared to treatment as usual (Stark et al., 2013).

Additional potentially clinical and medically relevant information was overlooked. When participants were asked about current treatment, one of the options was "no current treatment". Unfortunately, participants were not prompted to specify whether they were not receiving treatment because they were in remission or because they were in palliative care. This information would have been useful to provide a more accurate and nuanced understanding of the clinical sample. Number of lines of chemotherapy is also often used as a clinical marker of 
disease severity. The effects of second line chemotherapy on physical health have been mixed with some studies showing improvements in quality of life and symptoms compared to first line treatment (Bozcuk et al., 2006) and others showing minimal differences (Kiser, Greer, Wilmoth, Dmochowski, \& Naumann, 2010). Again, the absence of this information limits the clinical picture that forms of the present sample.

The present study made use of self-report measures of attachment and dyadic coping. The differences between self-reported dyadic coping and observed coping, such as "we" talk were addressed at previous points in the discussion. In sum, greater observed "we" talk demonstrated significant partner effects on affect and psychological distress among couples facing head and neck cancer (Badr et al., 2016) and was significantly associated with health outcomes in cardiac patients beyond a self-report measure of dyadic appraisal and dyadic coping (Rohrbaugh et al., 2008). Although an observation method may have provided further insight into the dyadic coping process, the Dyadic Coping Inventory is a reliable and comprehensive measure that is consistent and generalizable across the literature on dyadic coping (Bodenmann, 2005; Bodenmann et al., 2011). There are also observational measures available for adult attachment, such as the Adult Attachment Interview (AAI; Gillath, Karantzas, \& Fraley, 2016). Observational and self-report measures of attachment show little convergence (Gillath et al., 2016). Although the AAI may have provided a unique perspective, the AAI is more commonly used within the developmental literature whereas self-report measures, especially the ECR-R, are more commonly used within the social psychology and clinical health lines of research. Self-report questionnaires also have more direct applications within the clinical-health literature as they are more accessible for healthcare professionals to use. 
Finally, the present study was limited by the cross-sectional nature of its design.

Although a mediation model was tested, conclusions about causation cannot be interfered. For example, the current study reported significant effects of dyadic coping on well-being, however it is possible that greater well-being leads to better dyadic coping. As was noted in an aforementioned study in which change in marital satisfaction predicted change in attachment security (Benson et al., 2013), it is also possible that greater positive and less negative dyadic coping leads to greater attachment security over time, which in turn increases well-being. A longitudinal study design is needed in order to draw conclusions about causal conclusions about mechanisms of action.

\section{Future Directions}

The present study tested the model put forth by Pietromonaco and colleagues (2013) in which it was suggested that attachment negatively impacts health outcomes for couples by way of dyadic processes. Partial support was found as there were significant actor effects of attachment on health-related quality of life, and dyadic coping significantly mediated the association between attachment and social well-being. Future studies should evaluate the clinical extensions of these findings. For example, studies should examine whether interventions for dyadic coping such as Couples Coping Enhancement Training (Bodenmann \& Shantinath, 2004) have a significant effect on quality of life among couples coping with cancer. Additionally, the present study adds to the literature suggesting that attachment has important effects on clinical health outcomes; however, the literature is mixed regarding whether attachment should be considered a treatment target itself, or whether it is sufficient to target the thoughts and behaviours that result from the internal working models of attachment. An interesting future direction for the clinical attachment literature would be to examine the effects of treatments that 
target attachment directly compared to those addressing attachment indirectly through attachment-related thoughts and behaviours. Moreover, studies can examine whether these interventions that address the complex clinical picture that results from insecure attachment then have a significant indirect effect on attachment security.

Additionally, as dyadic coping only mediated one of four domains of quality of life, future studies should consider what other dyadic processes and dyadic outcomes account for the effects of attachment. For example, among couples coping with cancer, attachment has been associated with social support (Nissen, 2016), caregiving (Braun et al., 2012; Kim et al., 2008), and relationship satisfaction (Shields et al., 2000). These factors, as well as dyadic variables identified within the greater literature such as intimacy (Pielage, Luteijn, \& Arrindell, 2005) should be considered as other possible mechanisms to understanding the way in which attachment negatively affects health outcomes. Additionally, in spite of the model put forth by Pietromonaco and colleagues (2013), future studies can explore whether other factors beyond dyadic processes and outcomes account for the negative effects of attachment on health outcomes. For example, emotion regulation and maladaptive perfectionism are consistently associated with insecure attachment and have been shown to mediate the associations between attachment and depression (Garrison, Kahn, Miller, \& Sauer, 2014; Wei, Mallinckrodt, Russell, \& Abraham, 2004).

In sum, the present study examined the dyadic effects of attachment and dyadic coping on quality of life, revealing significant actor effects for both ovarian cancer patients and their spouses. Additionally, the present study tested the integrative model put forth by Pietromonaco and colleagues (2013) supporting the mediating role of positive and negative dyadic coping between attachment and social well-being. These findings uniquely contribute to the literature by 
examining health-related outcomes, examining actor and partner effects of both members of the dyad, evaluating a mediation model using Bodenmann's (2005) systemic-transactional conceptualization of dyadic coping, and using appropriate statistical methods for dyadic data analysis. Future studies should consider evaluating clinical extensions of this study, such as the efficacy of dyadic coping interventions on health outcomes within a cancer population, as well as consider alternative dyadic processes and outcomes that may account for the negative effects of attachment on quality of life. 


\title{
Appendix 1: Questionnaires
}

\section{Patient Demographic and Medical Questionnaire}

\author{
Study ID
}

Understanding the Psychological Well-Being of Individuals and Couples Facing Ovarian Cancer Today's Date:

1) Age:

2) With whom do you live? $\square$ Spouse/Partner Self $\quad \square$ Children Other

3) Relationship Status: $\quad \square$ Married/Partnered $\quad \square$ Separated $\quad \square$ Divorced $\square$ Widowed $\quad \square$ Single $\quad \square$ Other

4) If you are in a relationship, how long have you been with your spouse/partner?

5) Do you have any biological children? $\quad \square$ Yes $\quad \square$ No

If yes, how many?

6) Employment:

$\square$ Working full-time $\quad \square$ Working part-time $\quad \square$ Retired $\quad \square$ Disability $\quad \square$ Not Employed

7) What is/was your job title?

8) What is your average annual income?
a) $0-40,000$
b) $41,000-75,000$
c) $\geq 75,000$

9) Years of education:
a) High School
b) Some College/University
c) College/University degree
d) Graduate School

10) Ethnicity:
White
Black
Aboriginal/Native/Indigenous
Asian
Hispanic
Other 
Treatment related information

Date of first ovarian cancer diagnosis

What is the stage of your ovarian cancer?

Stage 1

Stage 2

Stage 3

Stage 4

Other (please specify)

Type of treatment at current time:

Surgery only

Chemotherapy only

Surgery and chemotherapy

Radiation therapy only

Surgery and Radiation therapy

Surgery, Radiation, and Chemotherapy

Not currently receiving treatment

PAST Type of treatment (not at the current time, but you have received in the past):

$\square$ Surgery only

Chemotherapy only

Surgery and chemotherapy

Radiation therapy only

$\square$ Surgery and Radiation therapy

Surgery, Radiation, and Chemotherapy

Not applicable

At what point in your treatment are you at the current time? (check one)

At time of initial diagnosis

During primary treatment of ovarian cancer (i.e., surgery/chemotherapy)

Within 6 months of completing your first treatment

Within 6 to 12 months of completing your first treatment

Greater than one year from completing your first treatment

After recurrence of cancer

Other (please specify) 
Have you ever received genetic testing to see if you have a mutation on the BRCA gene? $\square$ Yes $\quad \square$ No

If you have received testing, did you receive a positive test result for:

BRCA $1 \quad \square$ Yes $\square$ No

BRCA $2 \quad \square$ Yes $\square$ No

15) Please provide the following information about your cancer experience, including recurrences:

\begin{tabular}{|l|l|l|l|}
\hline $\begin{array}{c}\text { Type of Cancer You } \\
\text { Were Diagnosed With } \\
\text { (including recurrences) }\end{array}$ & Age of Diagnosis & $\begin{array}{c}\text { Date of } \\
\text { Diagnosis }\end{array}$ & $\begin{array}{c}\text { Type of } \\
\text { Treatment } \\
\text { Received }\end{array}$ \\
\hline 1. & & & \\
\hline 2. & & & \\
\hline 3. & & & \\
\hline 4. & & & \\
\hline 5. & & & \\
\hline
\end{tabular}

16) Has your mother ever been diagnosed with ANY type of cancer? $\quad \square$ Yes $\quad \square$ No If yes, please answer the following:

\begin{tabular}{|l|l|}
\hline \multicolumn{1}{|c|}{ Type(s) of Cancer Diagnosed With } & When was the diagnosis? \\
\hline 1. & \\
\hline 2. & \\
\hline 3. & \\
\hline 4. & \\
\hline 5. & \\
\hline
\end{tabular}

17) Has your father ever been diagnosed with ANY type of cancer? $\quad \square$ Yes $\quad \square$ No If yes, please answer the following:

\begin{tabular}{|l|l|}
\hline \multicolumn{1}{|c|}{ Type(s) of Cancer Diagnosed With } & When was the diagnosis? \\
\hline 1. & \\
\hline 2. & \\
\hline 3. & \\
\hline 4. & \\
\hline 5. & \\
\hline
\end{tabular}


19) Has a sibling ever been diagnosed with ANY type of cancer?

$\square$ Yes

$\square$ No

If yes, please answer the following:

\begin{tabular}{|l|c|c|}
\hline & $\begin{array}{c}\text { Type(s) of Cancer Diagnosed } \\
\text { With }\end{array}$ & When was the diagnosis? \\
\hline Sibling 1 & & \\
\hline Sibling 2 & & \\
\hline Sibling 3 & & \\
\hline Sibling 4 & & \\
\hline Sibling 5 & & \\
\hline Sibling 6 & & \\
\hline
\end{tabular}

20) Has your child ever been diagnosed with ANY type of cancer?

If yes, please answer the following:
$\square$ Yes
$\square$ No

\begin{tabular}{|l|l|l|}
\hline & $\begin{array}{c}\text { Type(s) of Cancer Diagnosed } \\
\text { With }\end{array}$ & When was the diagnosis? \\
\hline Child 1 & & \\
\hline Child 2 & & \\
\hline Child 3 & & \\
\hline Child 4 & & \\
\hline Child 5 & & \\
\hline
\end{tabular}




\section{Spouse Demographic Questionnaire}

Study ID

Understanding the Psychological Well-Being of Individuals and Couples Facing Ovarian Cancer Today's Date:

1) Age:

2) With whom do you live? $\square$ Spouse/Partner $\square$ Self $\quad \square$ Children

Other

3) Relationship Status: $\square$ Married/Partnered $\quad \square$ Separated $\quad \square$ Divorced Widowed $\square$ Single $\square$ Other

4) How long have you been with your spouse/partner?

5) Do you have any biological children? $\quad \square$ Yes $\quad \square$ No

If yes, how many?

6) Employment:

Working full-time $\quad \square$ Working part-time $\quad \square$ Retired $\quad \square$ Disability

$\square$ Not Employed

7) What is/was your job title?

8) What is your average annual income?

d) $0-40,000$

e) $41,000-75,000$

f) $\geq 75,000$

9) Years of education:

e) High School

f) Some College/University

g) College/University degree

h) Graduate School

10) Ethnicity:
White
Black
Aboriginal/Native/Indigenous
Asian
Hispanic
Other

11) Do you have any other chronic illnesses? $\square$

Hypertension

Diabetes

High BP 
High cholesterol $\quad \square$ Other

12) Has your mother ever been diagnosed with ANY type of cancer? $\quad \square$ Yes $\quad \square$ No If yes, please answer the following:

\begin{tabular}{|l|l|}
\hline \multicolumn{1}{|c|}{ Type(s) of Cancer Diagnosed With } & When was the diagnosis? \\
\hline 1. & \\
\hline 2. & \\
\hline 3. & \\
\hline 4. & \\
\hline 5. & \\
\hline
\end{tabular}

13) Has your father ever been diagnosed with ANY type of cancer? $\quad \square$ Yes $\quad \square$ No If yes, please answer the following:

\begin{tabular}{|l|l|}
\hline \multicolumn{1}{|c|}{ Type(s) of Cancer Diagnosed With } & When was the diagnosis? \\
\hline 1. & \\
\hline 2. & \\
\hline 3. & \\
\hline 4. & \\
\hline 5. & \\
\hline
\end{tabular}

14) Has a sibling ever been diagnosed with ANY type of cancer? $\quad \square$ Yes $\quad \square$ No If yes, please answer the following:

\begin{tabular}{|l|l|l|}
\hline & $\begin{array}{c}\text { Type(s) of Cancer Diagnosed } \\
\text { With }\end{array}$ & When was the diagnosis? \\
\hline Sibling 1 & & \\
\hline Sibling 2 & & \\
\hline Sibling 3 & & \\
\hline Sibling 4 & & \\
\hline Sibling 5 & & \\
\hline Sibling 6 & & \\
\hline
\end{tabular}

15) Has your child ever been diagnosed with ANY type of cancer? $\quad \square$ Yes $\quad \square$ No If yes, please answer the following: 


\begin{tabular}{|l|c|c|}
\hline & $\begin{array}{c}\text { Type(s) of Cancer Diagnosed } \\
\text { With }\end{array}$ & When was the diagnosis? \\
\hline Child 1 & & \\
\hline Child 2 & & \\
\hline Child 3 & & \\
\hline Child 4 & & \\
\hline Child 5 & & \\
\hline
\end{tabular}




\section{$\underline{\text { ECR-R }}$}

INSTRUCTIONS: The statements below concern how you feel in emotionally intimate relationships. We are interested in how you generally experience relationships, not just in what is happening in a current relationship. Respond to each statement by circling the appropriate number to indicate how much you agree or disagree with each statement.

\begin{tabular}{|l|c|c|c|c|c|c|c|}
\hline & $\begin{array}{l}\text { Disagree } \\
\text { Agree } \\
\text { Strongly } \\
\text { Strongly }\end{array}$ & \multicolumn{5}{|l}{ Meutral } \\
\hline $\begin{array}{l}\text { 1. I often worry that my partner doesn't } \\
\text { really love me }\end{array}$ & 1 & 2 & 3 & 4 & 5 & 6 & 7 \\
\hline $\begin{array}{l}\text { 2. I find it relatively easy to get close to } \\
\text { my partner }\end{array}$ & 1 & 2 & 3 & 4 & 5 & 6 & 7 \\
\hline $\begin{array}{l}\text { 3. I often worry that my partner will not } \\
\text { want to stay with me }\end{array}$ & 1 & 2 & 3 & 4 & 5 & 6 & 7 \\
\hline $\begin{array}{l}\text { 4. I find it difficult to allow myself to } \\
\text { depend on romantic partners }\end{array}$ & 1 & 2 & 3 & 4 & 5 & 6 & 7 \\
\hline $\begin{array}{l}\text { 5. I worry that romantic partners won't } \\
\text { care about me as much as I care about } \\
\text { them }\end{array}$ & 1 & 2 & 3 & 4 & 5 & 6 & 7 \\
\hline $\begin{array}{l}\text { 6. I often wish that my partner's feelings } \\
\text { for me were as strong as my feelings for } \\
\text { him or her }\end{array}$ & 1 & 2 & 3 & 4 & 5 & 6 & 7 \\
\hline $\begin{array}{l}\text { 7. I feel comfortable sharing my private } \\
\text { thoughts and feelings with my partner }\end{array}$ & 1 & 2 & 3 & 4 & 5 & 6 & 7 \\
\hline $\begin{array}{l}\text { 8. When I show my feelings for } \\
\text { romantic partners, I'm afraid that they } \\
\text { will not feel the same about me }\end{array}$ & 1 & 2 & 3 & 4 & 5 & 6 & 7 \\
\hline $\begin{array}{l}\text { 9. I'm afraid that I will lose my } \\
\text { partner's love }\end{array}$ & 1 & 2 & 3 & 4 & 5 & 6 & 7 \\
\hline $\begin{array}{l}\text { 10. I don't feel comfortable opening up } \\
\text { to romantic partners }\end{array}$ & 1 & 2 & 3 & 4 & 5 & 6 & 7 \\
\hline $\begin{array}{l}\text { 11. I rarely worry about my partner } \\
\text { leaving me }\end{array}$ & 1 & 2 & 3 & 4 & 5 & 6 & 7 \\
\hline $\begin{array}{l}\text { 12. I prefer not to show a partner how I } \\
\text { feel deep down }\end{array}$ & 1 & 2 & 3 & 4 & 5 & 6 & 7 \\
\hline $\begin{array}{l}\text { 13. I get uncomfortable when a romantic } \\
\text { partner wants to be very close }\end{array}$ & 1 & 2 & 3 & 4 & 5 & 6 & 7 \\
\hline $\begin{array}{l}\text { 14. When my partner is out of sight, I } \\
\text { worry that he or she might become } \\
\text { interested in someone else }\end{array}$ & 1 & 2 & 3 & 4 & 5 & 6 & 7 \\
\hline $\begin{array}{l}\text { 15. I worry a lot about my relationships } \\
\text { 16. I am very comfortable being close to } \\
\text { romantic partners }\end{array}$ & 1 & 2 & 3 & 4 & 5 & 6 & 7 \\
\hline
\end{tabular}




\begin{tabular}{|l|l|l|l|l|l|l|l|}
\hline $\begin{array}{l}\text { 17. I prefer not to be too close to } \\
\text { romantic partners }\end{array}$ & 1 & 2 & 3 & 4 & 5 & 6 & 7 \\
\hline $\begin{array}{l}\text { 18. It's not difficult for me to get close } \\
\text { to my partners }\end{array}$ & 1 & 2 & 3 & 4 & 5 & 6 & 7 \\
\hline
\end{tabular}




\section{Dyadic Coping Inventory (DCl) English Version (Bodenmann, 2007)}

This Scale is designed to measure how you and your partner cope with stress. Please respond spontaneously and as honestly as you can.

Please response to any item by marking the appropriate case, which is fitting to your personal situation.

There are no false answers.

This section is about how you communicate your stress to your partner.

1. I let my partner know that $\mathrm{I}$ appreciate his/her practical
2. I ask my partner to do things for me when I have too much to
do. I show my partner through my behaviour that I am not doing
well or when I have problems.

This section is about what your partner does when you are feeling stressed.

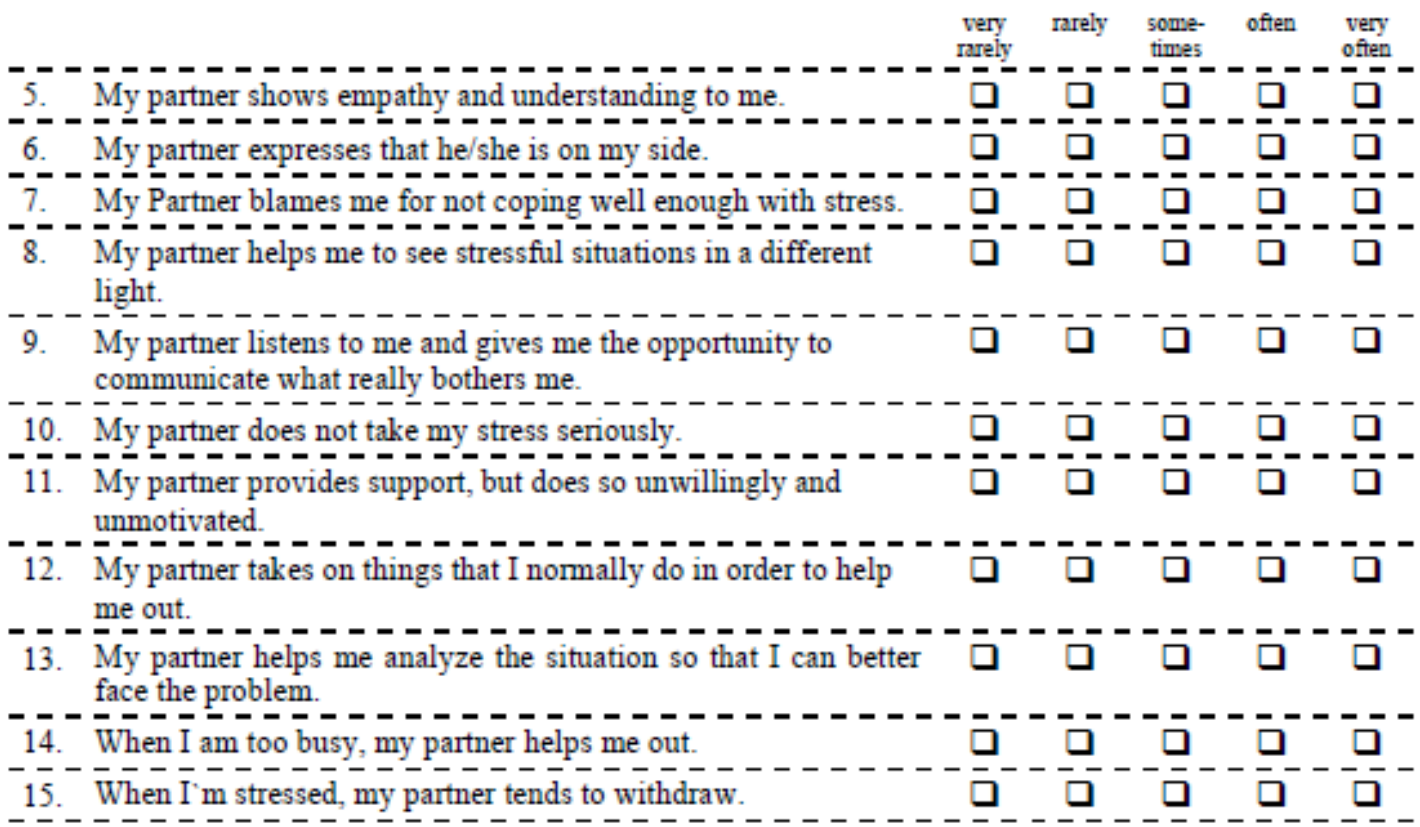


This section is about how your partner communicates when he/she is feeling stressed.

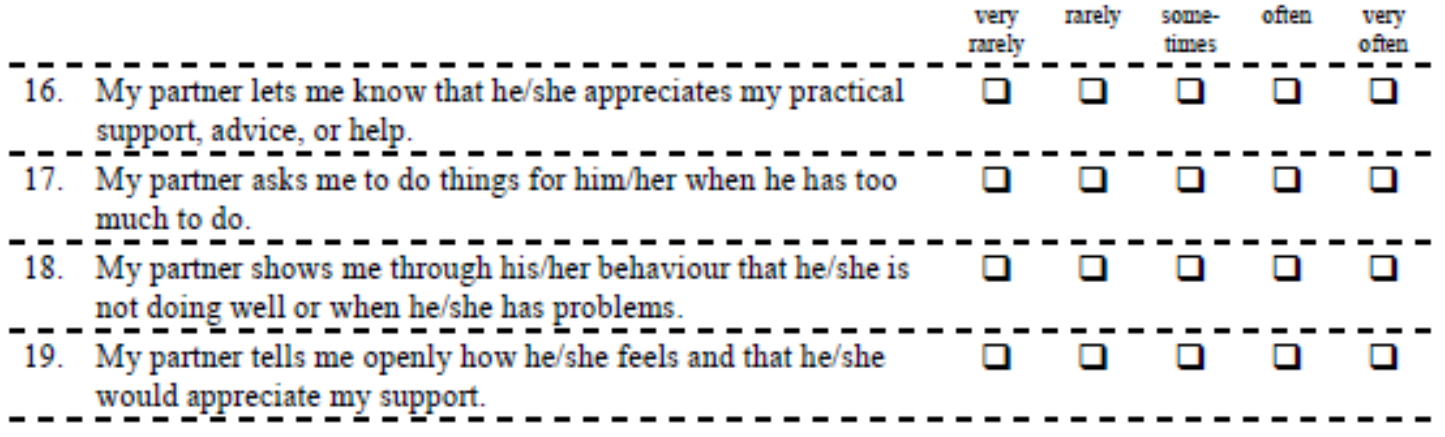

This section is about what you do when your partner makes know his/her stress.

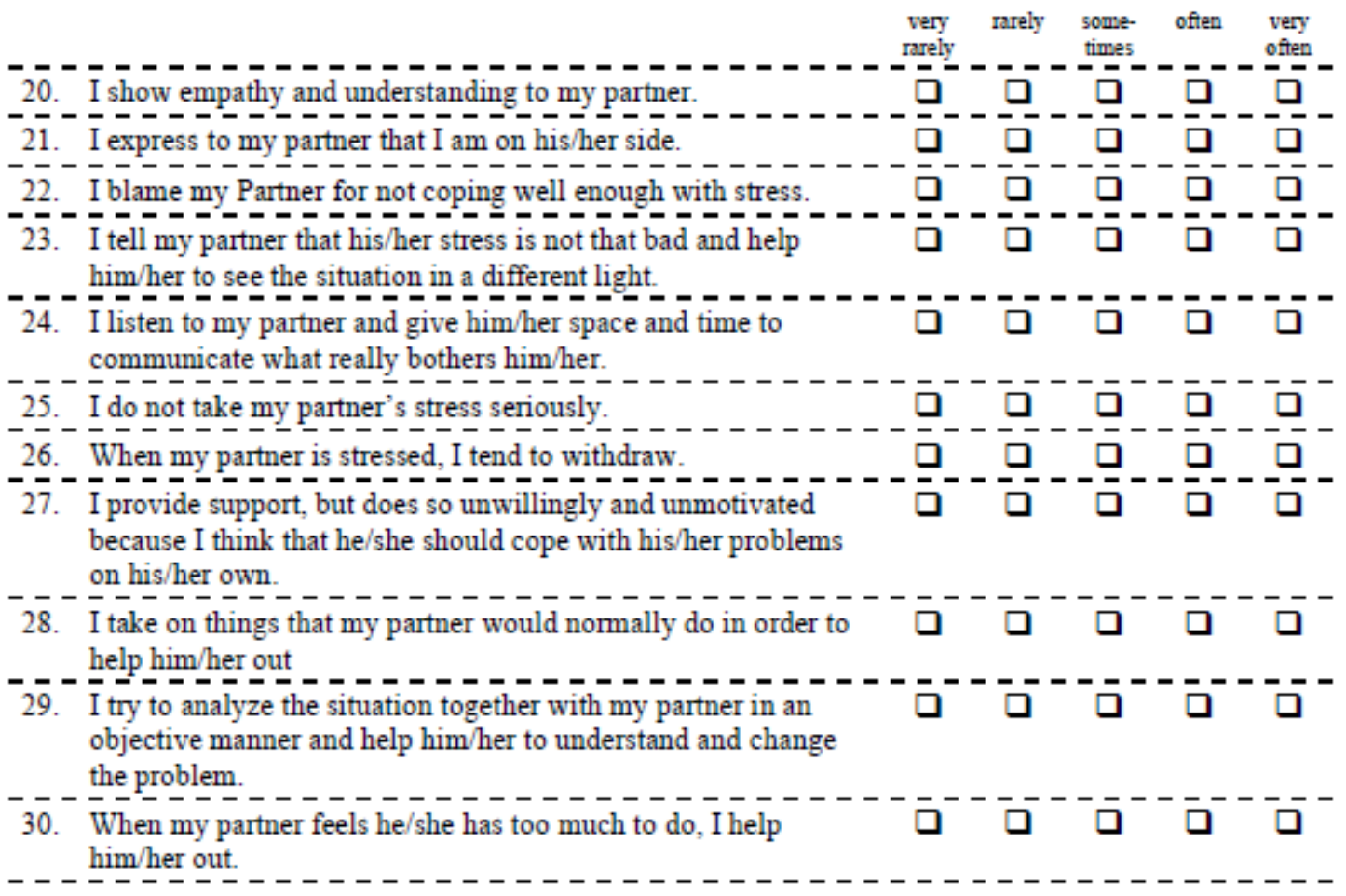


This section is about what you and your partner do when you are both feeling stressed.

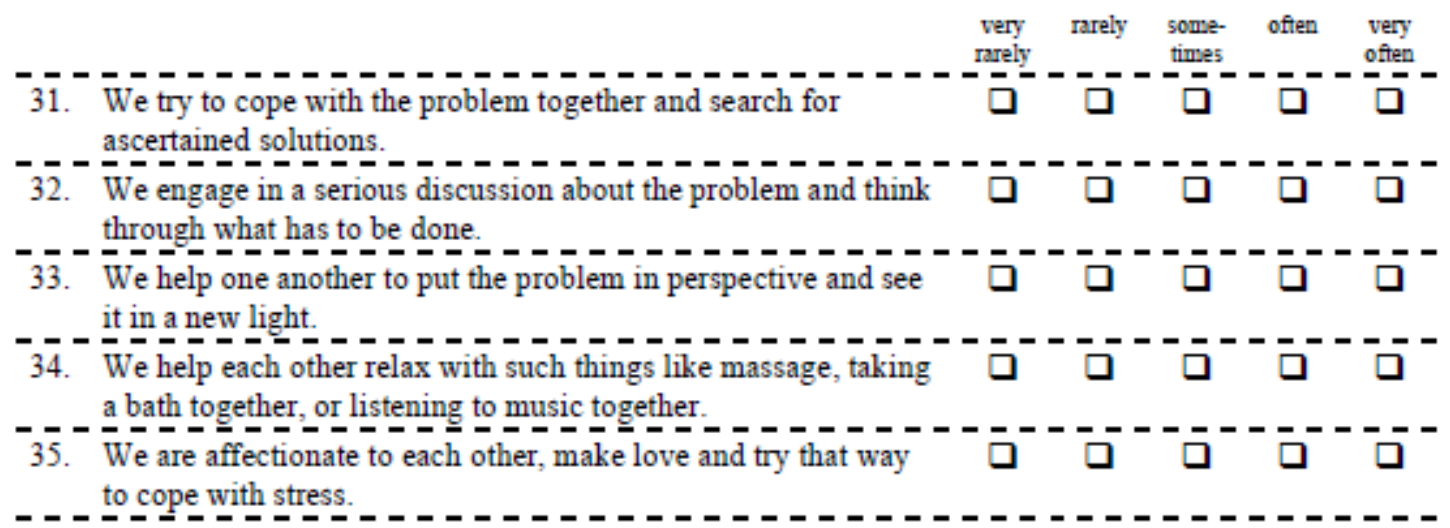

This section is about how you evaluate your coping as a couple.

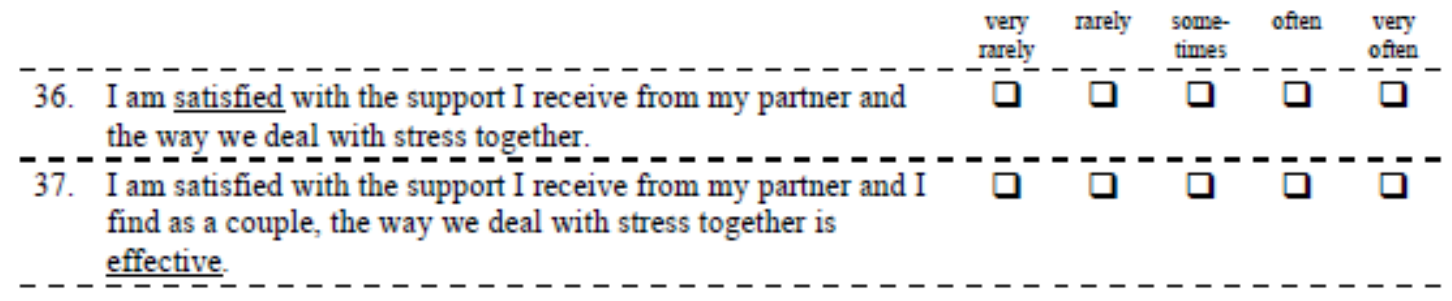




\section{FACT-O (Version 4)}

Below is a list of statements that other people with your illness have said are important. Please circle or mark one number per line to indicate your response as it applies to the past 7 days.

\section{PHYSICAL WELL-BEING}

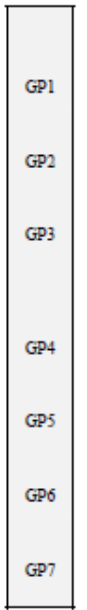

7

\section{SOCIAL/FAMILY WELL-BEING}

Because of my physical condition, I have trouble meeting the needs of my family

I have pain

I am bothered by side effects of treatment

I feel ill

I am forced to spend time in bed
I feel close to my friends

I get emotional support from my family

I get support from my friends

My family has accepted my illness

I am satisfied with family communication about my

illness.

I feel close to my partner (or the person who is my main support)

Regardless of your current level of sexual activity, please answer the following question. If you prefer not to answer it, please mark this box $\square$ and go to the next section.

I am satisfied with my sex life

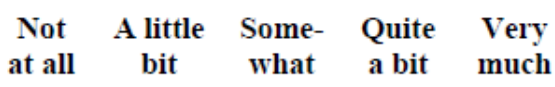

at all bit what a bit much

$\begin{array}{lllll}0 & 1 & 2 & 3 & 4 \\ 0 & 1 & 2 & 3 & 4\end{array}$

$\begin{array}{lllll}0 & 1 & 2 & 3 & 4\end{array}$

$\begin{array}{lllll}0 & 1 & 2 & 3 & 4\end{array}$

$\begin{array}{lllll}0 & 1 & 2 & 3 & 4\end{array}$

$\begin{array}{lllll}0 & 1 & 2 & 3 & 4\end{array}$

$\begin{array}{lllll}0 & 1 & 2 & 3 & 4\end{array}$

Not A little Some- Quite Very at all bit what a bit much 


\section{FACT-O (Version 4)}

Please circle or mark one number per line to indicate your response as it applies to the past 7 days.

\section{EMOTIONAL WELL-BEING}

I feel sad

I am satisfied with how I am coping with my illness

I am losing hope in the fight against my illness

I feel nervous

I worry about dying

I worry that my condition will get worse

\section{FUNCTIONAL WELL-BEING}

\begin{tabular}{|l|}
\hline GF1 \\
GF2 \\
GF3 \\
GF4 \\
GF5 \\
GF7
\end{tabular}

I am able to work (include work at home)

My work (include work at home) is fulfilling

I am able to enjoy life.

I have accepted my illness.

I am sleeping well

I am enjoying the things I usually do for fun

I am content with the quality of my life right now.

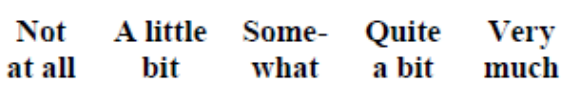

at all bit what a bit much

0

1

2

3

4

$\begin{array}{llll}1 & 2 & 3 & 4\end{array}$

0

1

$\begin{array}{lll}2 & 3 & 4\end{array}$

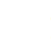

1

$\begin{array}{lll}2 & 3 & 4\end{array}$

1

1

2

1

23

4 


\section{FACT-O (Version 4)}

Please circle or mark one number per line to indicate your response as it applies to the past 7 days.

\section{ADDITIONAL CONCERNS}

\begin{tabular}{|c|}
\hline $\mathrm{O} 1$ \\
$\mathrm{C} 2$ \\
$\mathrm{C} 3$ \\
$\mathrm{O} 2$ \\
$\mathrm{~B} 5$ \\
$\mathrm{C} 6$ \\
$\mathrm{C} 7$ \\
$\mathrm{BMT} 5$ \\
$\mathrm{BL} 4$ \\
$\mathrm{~B} 9$ \\
$\mathrm{~B} 5$ \\
\end{tabular}

I have swelling in my stomach area

I am losing weight

I have control of my bowels

I have been vomiting .

I am bothered by hair loss

I have a good appetite

I like the appearance of my body

Not
at all

$\begin{array}{cccc}\text { A little } & \begin{array}{c}\text { Some- } \\ \text { bit }\end{array} & \begin{array}{c}\text { Quite } \\ \text { what }\end{array} \text { a bit } & \text { Very } \\ \text { much }\end{array}$

I am able to get around by myself...

I am able to feel like a woman .

I have cramps in my stomach area

I am interested in sex.

I have concerns about my ability to have children. 


\section{FACT-GP (Version 4)}

Below is a list of statements that other people have said are important. Please circle or mark one number per line to indicate your response as it applies to the past 7 davs.

\section{PHYSICAL WELL-BEING}

\begin{tabular}{|c|}
\hline GP1 \\
GP2 \\
GP3 \\
GP4 \\
GP6 \\
GP7 \\
\hline
\end{tabular}

I have a lack of energy

I have nausea meeting the needs of my family.....

I have pain

I feel ill

I am forced to spend time in bed......

Because of my physical condition, I have trouble

\section{SOCIAL/FAMILY WELL-BEING}

$\begin{array}{ccccc}\text { Not } & \text { A little } & \begin{array}{c}\text { Some- } \\ \text { at all }\end{array} \text { bit } & \begin{array}{c}\text { Quite } \\ \text { what }\end{array} & \begin{array}{c}\text { Very } \\ \text { a bit }\end{array} \\ \text { much }\end{array}$

4

0

1

2

3

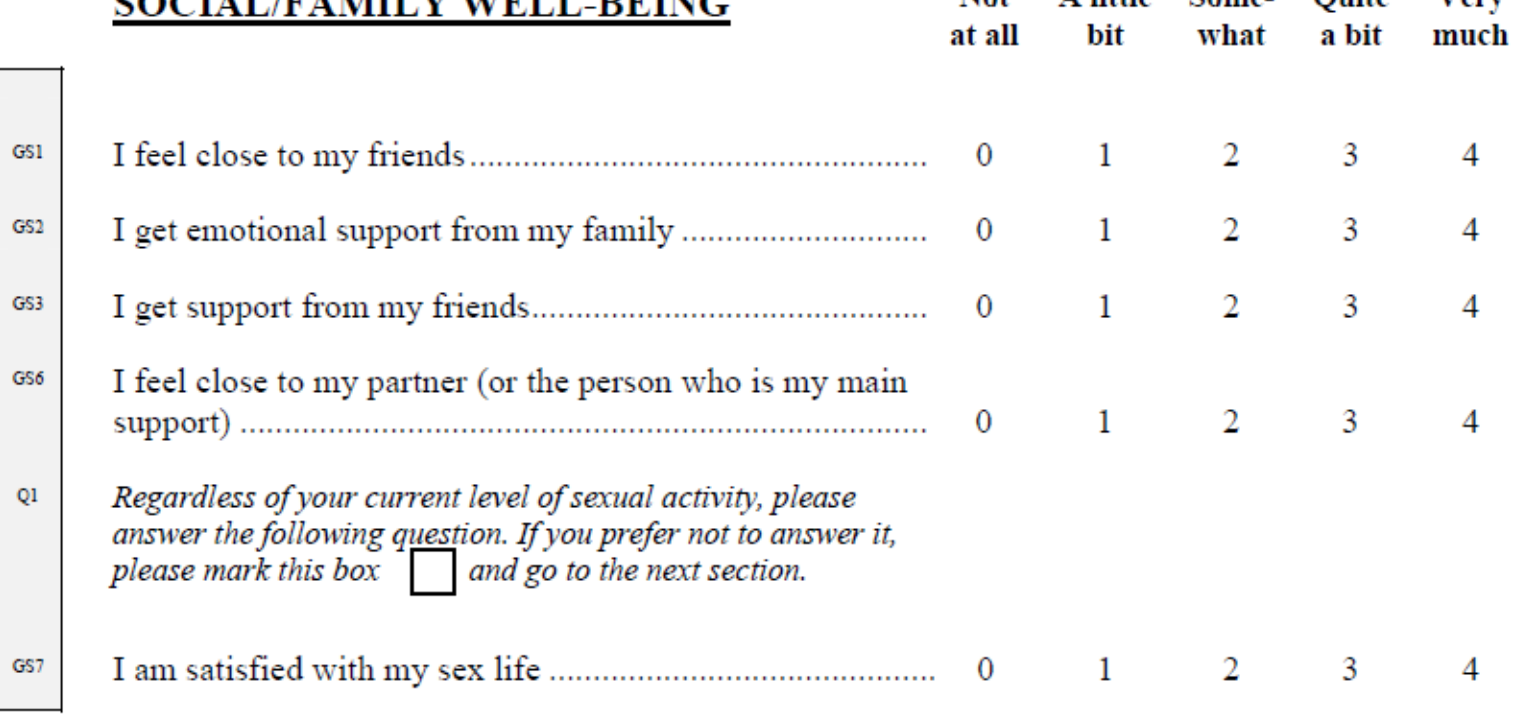




\section{FACT-GP (Version 4)}

Please circle or mark one number per line to indicate your response as it applies to the past 7 days.

\section{EMOTIONAL WELL-BEING}

\begin{tabular}{|l|}
\hline GE1 \\
GE4 \\
GE5 \\
GE6 \\
\hline
\end{tabular}

I feel sad

I feel nervous

I worry about dying

I worry that my condition will get worse

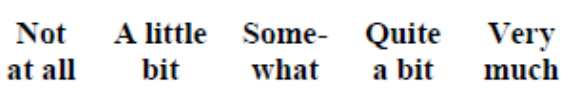

at all bit what a bit much

\section{FUNCTIONAL WELL-BEING}

\begin{tabular}{|l|}
\hline GF1 \\
GF2 \\
GF3 \\
GF5 \\
GF7
\end{tabular}

I am able to work (include work at home)

My work (include work at home) is fulfilling.

I am able to enjoy life

I am sleeping well

I am enjoying the things I usually do for fun

I am content with the quality of my life right now.

$\begin{array}{ccccc}\text { Not } & \text { A little } & \text { Some- } & \text { Quite } & \text { Very } \\ \text { at all } & \text { bit } & \begin{array}{c}\text { what } \\ \text { a bit }\end{array} & \text { much }\end{array}$




\section{References}

Aarts, F., Hinnen, C., Gerdes, V. E. A., Acherman, Y., \& Brandjes, D. P. M. (2014). Coping style as a mediator between attachment and mental and physical health in patients suffering from morbid obesity. International Journal of Psychiatry in Medicine, 47, 7591. https://doi.org/10.2190/PM.47.1.g

Arden-Close, E., Gidron, Y., Bayne, L., \& Moss-Morris, R. (2013). Written emotional disclosure for women with ovarian cancer and their partners: Randomised controlled trial. PsychoOncology, 22, 2262-2269. https://doi.org/10.1002/pon.3280

Ávila, M., Brandão, T., Teixeira, J., Coimbra, J. L., \& Matos, P. M. (2015). Attachment, emotion regulation, and adaptation to breast cancer: Assessment of a mediational hypothesis. Psycho-Oncology, 24, 1514-1520. https://doi.org/10.1002/pon.3817

Badr, H., Carmack, C. L., Kashy, D. A., Cristofanilli, M., \& Revenson, T. A. (2010). Dyadic coping in metastatic breast cancer. Health Psychology, 29, 169-180. https://doi.org/10.1037/a0018165

Badr, H., \& Krebs, P. (2013). A systematic review and meta-analysis of psychosocial interventions for couples coping with cancer. Psycho-Oncology, 22, 1688-1704. https://doi.org/10.1002/pon.3200

Badr, H., Milbury, K., Majeed, N., Carmack, C. L., Ahmad, Z., \& Gritz, E. R. (2016). Natural language use and couples' adjustment to head and neck cancer. Health Psychology. https://doi.org/10.1037/hea0000377

Banthia, R., Malcarne, V. L., Varni, J. W., Ko, C. M., Sadler, G. R., \& Greenbergs, H. L. (2003). The effects of dyadic strength and coping styles on psychological distress in couples 
faced with prostate cancer. Journal of Behavioral Medicine, 26, 31-52.

https://oi.org/10.1023/A:1021743005541

Basen-Engquist, K., Bodurka-Bevers, D., Fitzgerald, M. A., Webster, K., Cella, D., Hu, S., \&

Gershenson, D. M. (2001). Reliability and validity of the Functional Assessment of Cancer Therapy-Ovarian. Journal of Clinical Oncology, 19, 1809-1817.

Beck, J. (2011). Cognitive behavior therapy: Basics and beyond. New York, NY: Guilford Press.

Beck, L. A., Pietromonaco, P. R., DeVito, C. C., Powers, S. I., \& Boyle, A. M. (2014).

Congruence between spouses' perceptions and observers' ratings of responsiveness: The role of attachment avoidance. Personality and Social Psychology Bulletin, 40, 164-174. https://doi.org/10.1177/0146167213507779

Benson, L. A., Sevier, M., \& Christensen, A. (2013). The impact of behavioral couple therapy on attachment in distressed couples. Journal of Marital and Family Therapy, 39, 407-420. https://doi.org/10.1111/jmft.12020

Berg, C. A., \& Upchurch, R. (2007). A developmental-contextual model of couples coping with chronic illness across the adult life span. Psychological Bulletin, 133, 920-954. https://doi.org/10.1037/0033-2909.133.6.920

Berg, C. A., Wiebe, D. J., Butner, J., Bloor, L., Bradstreet, C., Upchurch, R., ... Patton, G. (2008). Collaborative coping and daily mood in couples dealing with prostate cancer. Psychology and Aging, 23, 505-516. https://doi.org/10.1037/a0012687

Bergelt, C., Koch, U., \& Petersen, C. (2008). Quality of life in partners of patients with cancer. Quality of Life Research, 17, 653-663. https://doi.org/10.1007/s11136-008-9349-y 
Bodenmann, G. (1997). Dyadic coping: A systemic-transactional view of stress and coping among couples: Theory and empirical findings. European Review of Applied Psychology / Revue Européenne de Psychologie Appliquée, 47, 137-141.

Bodenmann, G. (2005). Dyadic coping and its significance for marital functioning. In T. A. Revenson, K. Kayser, \& G. Bodenmann (Eds.), Couples coping with stress: Emerging perspectives on dyadic coping (pp. 33-49). Washington, DC, US: American Psychological Association.

Bodenmann, G., Charvoz, L., Cina, A., \& Widmer, K. (2001). Prevention of marital distress by enhancing the coping skills of couples: 1-year follow-up-study. Swiss Journal of Psychology / Schweizerische Zeitschrift Für Psychologie / Revue Suisse de Psychologie, 60,3-10. https://doi.org/10.1024//1421-0185.60.1.3

Bodenmann, G., Meuwly, N., \& Kayser, K. (2011). Two conceptualizations of dyadic coping and their potential for predicting relationship quality and individual well-being: A comparison. European Psychologist, 16, 255-266. https://doi.org/10.1027/1016$9040 / \mathrm{a} 000068$

Bodenmann, G., \& Shantinath, S. D. (2004). The Couples Coping Enhancement Training (CCET): A new approach to prevention of marital distress based upon stress and coping. Family Relations: An Interdisciplinary Journal of Applied Family Studies, 53, 477-484. https://doi.org/10.1111/j.0197-6664.2004.00056.x

Boehmer, S., Luszczynska, A., \& Schwarzer, R. (2007). Coping and quality of life after tumor surgery: Personal and social resources promote different domains of quality of life. Anxiety, Stress \& Coping: An International Journal, 20, 61-75. https://doi.org/10.1080/10615800701195439 
Bozcuk, H., Dalmis, B., Samur, M., Ozdogan, M., Artac, M., \& Savas, B. (2006). Quality of life in patients with advanced non-small cell lung cancer. Cancer Nursing, 29, 104-110. https://doi.org/10.1097/00002820-200603000-00004

Braun, M., Hales, S., Gilad, L., Mikulicer, M., Rydall, A., \& Rodin, G. (2012). Caregiving styles and attachment orientations in couples facing advanced cancer. Psycho-Oncology, 21, 935-943. https://doi.org/10.1002/pon.1988

Brucker, P. S., Yost, K., Cashy, J., Webster, K., \& Cella, D. (2005). General population and cancer patient norms for the Functional Assessment of Cancer Therapy-General (FACTG). Evaluation \& the Health Professions, 28, 192-211. https://doi.org/10.1177/0163278705275341

Campbell, L. C., Keefe, F. J., Scipio, C., McKee, D. C., Edwards, C. L., Herman, S. H., ... Donatucci, C. (2007). Facilitating research participation and improving quality of life for African American prostate cancer survivors and their intimate partners: A pilot study of telephone-based coping skills training. Cancer, 109, 414-424. https://doi.org/10.1002/cncr.22355

Canadian Cancer Society's Advisory Committee on Cancer Statistics. (2016). Canadian Cancer Statistics 2016. Canadian Cancer Society, Toronto, ON.

Cassidy, J., \& Shaver, P. R. (Eds.). (2008). Handbook of Attachment, Second Edition: Theory, Research, and Clinical Applications. New York, NY, US: Guilford Press.

Chandler, J. F. (2010). The use of social and temporal comparison to explore the process of quality of life evaluation in older adults. (Doctoral dissertation). Retrieved from ProQuest Information \& Learning 
Chopik, W. J., Edelstein, R. S., \& Fraley, R. C. (2013). From the cradle to the grave: Age differences in attachment from early adulthood to old age. Journal of Personality, 81, 171-183. https://doi.org/10.1111/j.1467-6494.2012.00793.x

Ciechanowski, P. S., Walker, E. A., Katon, W. J., \& Russo, J. E. (2002). Attachment theory: A model for health care utilization and somatization. Psychosomatic Medicine, 64, 660667. https://doi.org/10.1097/01.PSY.0000021948.90613.76

Collins, N. L., \& Feeney, B. C. (2004). Working models of attachment shape perceptions of social support: Evidence from experimental and observational studies. Journal of Personality and Social Psychology, 87, 363-383. https://doi.org/10.1037/00223514.87.3.363

Dagan, M., \& Hagedoorn, M. (2014). Response rates in studies of couples coping with cancer: A systematic review. Health Psychology, 33, 845-852. https://doi.org/10.1037/hea0000013

Doherty, N. A., \& Feeney, J. A. (2004). The composition of attachment networks throughout the adult years. Personal Relationships, 11, 469-488. https://doi.org/10.1111/j.14756811.2004.00093.x

Feldman, B. N., \& Broussard, C. A. (2005). The influence of relational factors on men's adjustment to their partners' newly-diagnosed breast cancer. Journal of Psychosocial Oncology, 23, 23-43. https://doi.org/10.1300/J077v23n02_03

Feldman, B. N., \& Broussard, C. A. (2006). Men's adjustment to their partners' breast cancer: A dyadic coping perspective. Health \& Social Work, 31, 117-127. https://doi.org/10.1093/hsw/31.2.117 
Foster, C., Myall, M., Scott, I., Sayers, M., Brindle, L., Cotterell, P., ... Robinson, J. (2015). "You can't say, “what about me?" I'm not the one with cancer': Information and support needs of relatives. Psycho - Oncology, 24, 705. https://doi.org/10.1002/pon.3716

Fraley, R. C., Waller, N. G., \& Brennan, K. A. (2000). An item response theory analysis of selfreport measures of adult attachment. Journal of Personality and Social Psychology, 78, $350-365$.

Frost, M. H., Johnson, M. E., Atherton, P. J., Petersen, W. O., Dose, A. M., Kasner, M. J., ... Pipe, T. B. (2012). Spiritual well-being and quality of life of women with ovarian cancer and their spouses. The Journal of Supportive Oncology, 10, 72-80. https://doi.org/10.1016/j.suponc.2011.09.001

Fuenfhausen, K. K., \& Cashwell, C. S. (2013). Attachment, stress, dyadic coping, and marital satisfaction of counseling graduate students. Family Journal, 21, 364.

Garrison, A. M., Kahn, J. H., Miller, S. A., \& Sauer, E. M. (2014). Emotional avoidance and rumination as mediators of the relation between adult attachment and emotional disclosure. Personality and Individual Differences, 70, 239-245. https://doi.org/10.1016/j.paid.2014.07.006

Gillath, O., Karantzas, G. C., \& Fraley, R. C. (2016). Adult attachment: A concise introduction to theory and research. Academic Press.

Givertz, M., Woszidlo, A., Segrin, C., \& Knutson, K. (2013). Direct and indirect effects of attachment orientation on relationship quality and loneliness in married couples. Journal of Social and Personal Relationships, 30, 1096-1120. https://doi.org/10.1177/0265407513482445 
Gonçalves, V. (2010). Long-term quality of life in gynecological cancer survivors. Current Opinion in Obstetrics \& Gynecology, 22, 30-35. https://doi.org/10.1097/GCO.0b013e328332e626

Hagedoorn, M., Buunk, B. P., Kuijer, R. G., Wobbes, T., \& Sanderman, R. (2000). Couples dealing with cancer: Role and gender differences regarding psychological distress and quality of life. Psycho-Oncology, 9, 232-242. https://doi.org/10.1002/1099-

1611(200005/06)9:3<232::AID-PON458>3.0.CO;2-J

Häusler, A., Sánchez, A., Gellert, P., Deeken, F., Rapp, M. A., \& Nordheim, J. (2016). Perceived stress and quality of life in dementia patients and their caregiving spouses: Does dyadic coping matter? International Psychogeriatrics.

https://doi.org/10.1017/S1041610216001046

Hill, E. M. (2016). Quality of life and mental health among women with ovarian cancer: Examining the role of emotional and instrumental social support seeking. Psychology, Health \& Medicine, 21, 551-561. https://doi.org/10.1080/13548506.2015.1109674

Hinnen, C., Ranchor, A. V., Sanderman, R., Snijders, T. A. B., Hagedoorn, M., \& Coyne, J. C. (2008). Course of distress in breast cancer patients, their partners, and matched control couples. Annals of Behavioral Medicine., 36, 141-148. https://doi.org/10.1007/s12160008-9061-8

Hsiao, F.-H., Jow, G.-M., Kuo, W.-H., Huang, C.-S., Lai, Y.-M., Liu, Y.-F., \& Chang, K.-J. (2014). The partner's insecure attachment, depression and psychological well-being as predictors of diurnal cortisol patterns for breast cancer survivors and their spouses. Stress: The International Journal on the Biology of Stress, 17, 169-175. https://doi.org/10.3109/10253890.2014.880833 
Hsieh, C.-C., Chen, C.-A., Hsiao, F.-H., \& Shun, S.-C. (2014). The correlations of sexual activity, sleep problems, emotional distress, attachment styles with quality of life: Comparison between gynaecological cancer survivors and noncancer women. Journal of Clinical Nursing, 23, 985-994. https://doi.org/10.1111/jocn.12232

Hunter, J. J., \& Maunder, R. G. (2001). Using attachment theory to understand illness behavior. General Hospital Psychiatry, 23, 177-182. https://doi.org/10.1016/S01638343(01)00141-4

Johnson, M. D., Anderson, J. R., Walker, A., Wilcox, A., Lewis, V. L., \& Robbins, D. C. (2013). Common dyadic coping is indirectly related to dietary and exercise adherence via patient and partner diabetes efficacy. Journal of Family Psychology, 27, 722-730. https://doi.org/10.1037/a0034006

Kafetsios, K., \& Sideridis, G. D. (2006). Attachment, social support and well-being in young and older adults. Journal of Health Psychology, 11, 863-875. https://doi.org/10.1177/1359105306069084

Kardatzke, K. N. (2010). Perceived stress, adult attachment, dyadic coping, and marital satisfaction of counseling graduate students. Retrieved from ProQuest Information \& Learning

Kayser, K., \& Sormanti, M. (2002). A follow-up study of women with cancer: Their psychosocial well-being and close relationships. Social Work in Health Care, 35, 391406.

Kayser, K., Watson, L. E., \& Andrade, J. T. (2007). Cancer as a "we-disease": Examining the process of coping from a relational perspective. Families, Systems, \& Health, 25, 404418. https://doi.org/10.1037/1091-7527.25.4.404 
Kenny, D. A., Kashy, D. A., \& Cook, W. L. (2006). Dyadic data analysis. New York, NY: Guilford Press.

Kershaw, T., Ellis, K. R., Yoon, H., Schafenacker, A., Katapodi, M., \& Northouse, L. (2015). The interdependence of advanced cancer patients' and their family caregivers' mental health, physical health, and self-efficacy over time. Annals of Behavioral Medicine, 49, 901-911. https://doi.org/10.1007/s12160-015-9743-y

Kershaw, T., Mood, D. W., Newth, G., Ronis, D. L., Sanda, M. G., Vaishampayan, U., \& Northouse, L. L. (2008). Longitudinal analysis of a model to predict quality of life in prostate cancer patients and their spouses. Annals of Behavioral Medicine, 36, 117-128. https://doi.org/10.1007/s12160-008-9058-3

Kershaw, T., Northouse, L., Kritpracha, C., Schafenacker, A., \& Mood, D. (2004). Coping strategies and quality of life in women with advanced breast cancer and their family caregivers. Psychology \& Health, 19, 139-155. https://doi.org/10.1080/08870440310001652687

Kim, J., Han, J. Y., Shaw, B., McTavish, F., \& Gustafson, D. (2010). The roles of social support and coping strategies in predicting breast cancer patients' emotional well-being: Testing mediation and moderation models. Journal of Health Psychology, 15, 543-552. https://doi.org/10.1177/1359105309355338

Kim, Y., \& Carver, C. S. (2007). Frequency and difficulty in caregiving among spouses of individuals with cancer: Effects of adult attachment and gender. Psycho-Oncology, 16, 714-723. https://doi.org/10.1002/pon.1110

Kim, Y., Kashy, D. A., Wellisch, D. K., Spillers, R. L., Kaw, C. K., \& Smith, T. G. (2008). Quality of life in couples dealing with cancer: Dyadic and individual adjustment among 
breast and prostate cancer survivors and their spousal caregivers. Annals of Behavioral Medicine, 35, 230-238. https://doi.org/10.1007/s12160-008-9026-y

Kiser, D. W., Greer, T. B., Wilmoth, M. C., Dmochowski, J., \& Naumann, R. W. (2010). Peripheral neuropathy in patients with gynecologic cancer receiving chemotherapy: Patient reports and provider assessments. Oncology Nursing Forum, 37, 758-764. https://doi.org/10.1188/10.ONF.758-764

Lafaye, A., Petit, S., Richaud, P., Houédé, N., Baguet, F., \& Cousson-Gélie, F. (2014). Dyadic effects of coping strategies on emotional state and quality of life in prostate cancer patients and their spouses. Psycho-Oncology, 23, 797-803. https://doi.org/10.1002/pon.3483

Ledermann, T., Bodenmann, G., Gagliardi, S., Charvoz, L., Verardi, S., Rossier, J., ... Iafrate, R. (2010). Psychometrics of the Dyadic Coping Inventory in three language groups. Swiss Journal of Psychology/Schweizerische Zeitschrift Für Psychologie/Revue Suisse de Psychologie, 69, 201-212. https://doi.org/10.1024/1421-0185/a000024

Ledermann, T., Macho, S., \& Kenny, D. A. (2011). Assessing mediation in dyadic data using the actor-partner interdependence model. Structural Equation Modeling: A Multidisciplinary Journal, 18, 595-612. https://doi.org/10.1080/10705511.2011.607099

Levey, S. B. (2003). The effects of dyadic coping on the relationship between attachment style and relationship satisfaction. Dissertation Abstracts International: Section B: The Sciences and Engineering, 64, 2393.

Li, T., \& Fung, H. H. (2014). How avoidant attachment influences subjective well-being: An investigation about the age and gender differences. Aging \& Mental Health, 18, 4-10. https://doi.org/10.1080/13607863.2013.775639 
Litzelman, K., Green, P. A., \& Yabroff, K. R. (2016). Cancer and quality of life in spousal dyads: spillover in couples with and without cancer-related health problems. Supportive Care in Cancer, 24, 763-771. https://doi.org/http://dx.doi.org/10.1007/s00520-015-28404

Lockwood-Rayermann, S. (2006). Survivorship issues in ovarian cancer: A review. Oncology Nursing Forum, 33, 553-562. https://doi.org/10.1188/06.ONF.553-562

Malik, S., Wells, A., \& Wittkowski, A. (2015). Emotion regulation as a mediator in the relationship between attachment and depressive symptomatology: A systematic review. Journal of Affective Disorders, 172, 428-444. https://doi.org/10.1016/j.jad.2014.10.007

Manne, S., \& Badr, H. (2010). Intimacy processes and psychological distress among couples coping with head and neck or lung cancers. Psycho-Oncology., 19, 941-954. https://doi.org/10.1002/pon.1645

Manne, S., Myers, S., Ozga, M., Kissane, D., Kashy, D., Rubin, S., ... Rosenblum, N. (2014). Holding back sharing concerns, dispositional emotional expressivity, perceived unsupportive responses and distress among women newly diagnosed with gynecological cancers. General Hospital Psychiatry, 36, 81-87. https://doi.org/10.1016/j.genhosppsych.2013.10.001

Manne, S., Siegel, S. D., Heckman, C. J., \& Kashy, D. A. (2016). A randomized clinical trial of a supportive versus a skill-based couple-focused group intervention for breast cancer patients. Journal of Consulting and Clinical Psychology, 84, 668-681. https://doi.org/10.1037/ccp0000110 
Martin, L. A., Vosvick, M., \& Riggs, S. A. (2012). Attachment, forgiveness, and physical health quality of life in HIV + adults. AIDS Care, 24, 1333-1340.

https://doi.org/10.1080/09540121.2011.648598

Maunder, R. G., Lancee, W. J., Nolan, R. P., Hunter, J. J., \& Tannenbaum, D. W. (2006). The relationship of attachment insecurity to subjective stress and autonomic function during standardized acute stress in healthy adults. Journal of Psychosomatic Research, 60(3), 283-290. https://doi.org/10.1016/j.jpsychores.2005.08.013

McBride, C., \& Atkinson, L. (2009). Attachment theory and cognitive-behavioral therapy. In J. H. Obegi \& E. Berant (Eds.), Attachment Theory and Research in Clinical Work With Adults (pp. 434-458). New York, NY: Guilford Press.

McCarthy, C. J., Lambert, R. G., \& Moller, N. P. (2006). Preventive resources and emotion regulation expectancies as mediators between attachment and college students'stress outcomes. International Journal of Stress Management, 13, 1-22. https://doi.org/10.1037/1072-5245.13.1.1

Meier, C., Bodenmann, G., Moergeli, H., Peter-Wight, M., Martin, M., Buechi, S., \& Jenewein, J. (2012). Dyadic coping among couples with COPD: A pilot study. Journal of Clinical Psychology in Medical Settings, 19, 243-254. https://doi.org/10.1007/s10880-011-9279-7

Meredith, P., Strong, J., \& Feeney, J. A. (2006). Adult attachment, anxiety, and pain self-efficacy as predictors of pain intensity and disability. Pain, 123, 146-154. https://doi.org/10.1016/j.pain.2006.02.025

Merz L., E., Malcarne, V. L., Ko, C. M., Sadler, M., Kwack, L., Varni, J. W., \& Sadler, G. R. (2011). Dyadic concordance among prostate cancer patients and their partners and health- 
related quality of life: Does it matter? Psychology \& Health, 26, 651-666.

https://doi.org/10.1080/08870441003721251

Millings, A., \& Carnelley, K. B. (2015). Core belief content examined in a large sample of patients using online cognitive behaviour therapy. Journal of Affective Disorders, 186, 275-283. https://doi.org/10.1016/j.jad.2015.06.044

Millings, A., \& Walsh, J. (2009). A dyadic exploration of attachment and caregiving in longterm couples. Personal Relationships, 16, 437-453. https://doi.org/10.1111/j.14756811.2009.01232.x

Monin, J. K., Zhou, L., \& Kershaw, T. (2014). Attachment and psychological health in older couples coping with pain. GeroPsych: The Journal of Gerontopsychology and Geriatric Psychiatry, 27, 115-127. https://doi.org/10.1024/1662-9647/a000110

Moreira, H., \& Canavarro, M. C. (2013). Psychosocial adjustment and marital intimacy among partners of patients with breast cancer: A comparison study with partners of healthy women. Journal of Psychosocial Oncology, 31, 282-304.

https://doi.org/10.1080/07347332.2013.778934

Moser, M. B., Johnson, S. M., Dalgleish, T. L., Lafontaine, M.-F., Wiebe, S. A., \& Tasca, G. A. (2016). Changes in relationship-specific attachment in emotionally focused couple therapy. Journal of Marital and Family Therapy, 42, 231-245. https://doi.org/10.1111/jmft.12139

Mosher, C. E., Bakas, T., \& Champion, V. L. (2013). Physical healthy mental health and life changes among family caregivers of patients with lung cancer. Oncology Nursing Forum, 40, 53-61. https://doi.org/10.1188/13.ONF.53-61 
Naaman, S., Radwan, K., \& Johnson, S. (2009). Coping with early breast cancer: Couple adjustment processes and couple-based intervention. Psychiatry: Interpersonal and Biological Processes, 72, 321-345.

Nissen, K. G. (2016). Correlates of self-rated attachment in patients with cancer and their caregivers: A systematic review and meta-analysis. Psycho-Oncology. https://doi.org/10.1002/pon.4057

Pasek, M., Suchocka, L., \& Urbański, K. (2013). Quality of life in cervical cancer patients treated with radiation therapy. Journal of Clinical Nursing, 22, 690-697.

Pielage, S. B., Luteijn, F., \& Arrindell, W. A. (2005). Adult attachment, intimacy and psychological distress in a clinical and community sample. Clinical Psychology \& Psychotherapy, 12, 455-464. https://doi.org/10.1002/cpp.472

Pietromonaco, P. R., Uchino, B., \& Dunkel Schetter, C. (2013). Close relationship processes and health: Implications of attachment theory for health and disease. Health Psychology, 32, 499-513. https://doi.org/10.1037/a0029349

Platts, H., Tyson, M., \& Mason, O. (2002). Adult attachment style and core beliefs: Are they linked? Clinical Psychology \& Psychotherapy, 9, 332-348. https://doi.org/10.1002/cpp.345

Porter, L. S., Keefe, F. J., Davis, D., Rumble, M., Scipio, C., \& Garst, J. (2012). Attachment styles in patients with lung cancer and their spouses: Associations with patient and spouse adjustment. Supportive Care in Cancer, 20, 2459-66. https://doi.org/10.1007/s00520-011-1367-6

Rannestad, T., \& Skjeldestad, F. E. (2007). Pain and quality of life among long-term gynecological cancer survivors: A population-based case-control study. Acta Obstetricia 
et Gynecologica Scandinavica, 86, 1510-1516.

https://doi.org/10.1080/00016340701743116

Ravitz, P., Maunder, R., Hunter, J., Sthankiya, B., \& Lancee, W. (2010). Adult attachment measures: A 25-year review. Journal of Psychosomatic Research, 69, 419-432. https://doi.org/10.1016/j.jpsychores.2009.08.006

Regan, T. W., Lambert, S. D., Kelly, B., McElduff, P., Girgis, A., Kayser, K., \& Turner, J. (2014). Cross-sectional relationships between dyadic coping and anxiety, depression, and relationship satisfaction for patients with prostate cancer and their spouses. Patient Education and Counseling, 96, 120-127. https://doi.org/10.1016/j.pec.2014.04.010

Reisine, S., Fifield, J., \& Winkelman, D. K. (2000). Characteristics of rheumatoid arthritis patients: Who participates in long-term research and who drops out? Arthritis Care \& Research, 13, 3-10. https://doi.org/10.1002/1529-0131(200002)13:1

Robinson, K. M., Christensen, K. B., Ottesen, B., \& Krasnik, A. (2012). Diagnostic delay, quality of life and patient satisfaction among women diagnosed with endometrial or ovarian cancer: A nationwide Danish study. Quality of Life Research, 21, 1519-25. https://doi.org/10.1007/s11136-011-0077-3

Robles, T. F., Brooks, K. P., Kane, H. S., \& Schetter, C. D. (2013). Attachment, skin deep? Relationships between adult attachment and skin barrier recovery. International Journal of Psychophysiology, 88, 241-252. https://doi.org/10.1016/j.ijpsycho.2012.04.007

Rohrbaugh, M. J., Mehl, M. R., Shoham, V., Reilly, E. S., \& Ewy, G. A. (2008). Prognostic significance of spouse we talk in couples coping with heart failure. Journal of Consulting and Clinical Psychology, 76, 781-789. https://doi.org/10.1037/a0013238 
Rohrbaugh, M. J., Shoham, V., Skoyen, J. A., Jensen, M., \& Mehl, M. R. (2012). We-talk, communal coping, and cessation success in a couple-focused intervention for healthcompromised smokers. Family Process, 51, 107-121. https://doi.org/10.1111/j.1545$5300.2012 .01388 . x$

Roland, K. B., Rodriguez, J. L., Patterson, J. R., \& Trivers, K. F. (2013). A literature review of the social and psychological needs of ovarian cancer survivors. Psycho-Oncology, 22, 2408-2418. https://doi.org/10.1002/pon.3322

Rottmann, N., Hansen, D. G., Larsen, P. V., Nicolaisen, A., Flyger, H., Johansen, C., \& Hagedoorn, M. (2015). Dyadic coping within couples dealing with breast cancer: A longitudinal, population-based study. Health Psychology, 34, 486-495. https://doi.org/10.1037/hea0000218

Sammarco, A. (2009). Quality of life of breast cancer survivors: A comparative study of age cohorts. Cancer Nursing, 32, 347-356. https://doi.org/10.1097/NCC.0b013e31819e23b7

Schmidt, S., Nachtigall, C., Wuethrich-Martone, O., \& Strauss, B. (2002). Attachment and coping with chronic disease. Journal of Psychosomatic Research, 53, 763-773. https://doi.org/10.1016/S0022-3999(02)00335-5

Scott, J. L., Halford, K. W., \& Ward, B. G. (2004). United we stand? The effects of a couplecoping intervention on adjustment to early stage breast or gynecological cancer. Journal of Consulting and Clinical Psychology, 72, 1122-1135. https://doi.org/10.1037/0022006X.72.6.1122

Segrin, C., Badger, T. A., Meek, P., Lopez, A. M., Bonham, E., \& Sieger, A. (2005). Dyadic interdependence on affect and quality-of-life trajectories among women with breast 
cancer and their partners. Journal of Social and Personal Relationships, 22, 673-689. https://doi.org/10.1177/0265407505056443

Shaver, P. R., \& Mikulincer, M. (2008). Adult attachment and cognitive and affective reactions to positive and negative events. Social and Personality Psychology Compass, 2, 1844 1865. https://doi.org/10.1111/j.1751-9004.2008.00146.x

Shaver, P. R., \& Mikulincer, M. (2011). An attachment-theory framework for conceptualizing interpersonal behavior. In Handbook of interpersonal psychology: Theory, research, assessment, and therapeutic interventions. (pp. 17-35). Hoboken, NJ, USA: John Wiley \& Sons Inc.

Shields, C. G., Travis, L. A., \& Rousseau, S. L. (2000). Marital attachment and adjustment in older couples coping with cancer. Aging \& Mental Health, 4, 223-233. https://doi.org/10.1080/713649921

Shor, V., Grinstein-Cohen, O., Reinshtein, J., Liberman, O., \& Delbar, V. (2015). Health-related quality of life and sense of coherence among partners of women with breast cancer in Israel. European Journal of Oncology Nursing, 19, 18-22. https://doi.org/10.1016/j.ejon.2014.08.004

Sibley, C. G., Fischer, R., \& Liu, J. H. (2005). Reliability and validity of the revised Experiences in Close Relationships (ECR-R) self-report measure of adult romantic attachment. Personality and Social Psychology Bulletin, 31, 1524-1536. https://doi.org/10.1177/0146167205276865

Song, L., Northouse, L. L., Braun, T. M., Zhang, L., Cimprich, B., Ronis, D. L., \& Mood, D. W. (2011). Assessing longitudinal quality of life in prostate cancer patients and their 
spouses: A multilevel modeling approach. Quality of Life Research, 20, 371-381. https://doi.org/10.1007/s11136-010-9753-y

Stark, D., Nankivell, M., Pujade-Lauraine, E., Kristensen, G., Elit, L., Stockler, M., ... Perren, T. (2013). Standard chemotherapy with or without bevacizumab in advanced ovarian cancer: Quality-of-life outcomes from the International Collaboration on Ovarian Neoplasms (ICON7) phase 3 randomised trial. The Lancet, Oncology, 14, 236-243. https://doi.org/10.1016/S1470-2045(12)70567-3

Stoppa-Lyonnet, D. (2016). The biological effects and clinical implications of BRCA mutations: Where do we go from here? European Journal of Human Genetics: EJHG, 24, S3-9. https://doi.org/10.1038/ejhg.2016.93

Taylor, P., Rietzschel, J., Danquah, A., \& Berry, K. (2015). Changes in attachment representations during psychological therapy. Psychotherapy Research, 25, 222-238. https://doi.org/10.1080/10503307.2014.886791

Vacek, P. M., Winstead-Fry, P., Secker-Walker, R. H., Hooper, G. J., \& Plante, D. A. (2003). Factors influencing quality of life in breast cancer survivors. Quality of Life Research: An International Journal of Quality of Life Aspects of Treatment, Care \& Rehabilitation, 12, 527-537. https://doi.org/10.1023/A:1025098108717

Vaske, I., Thöne, M. F., Kühl, K., Keil, D. C., Schürmann, W., Rief, W., \& Stenzel, N. M. (2015). For better or for worse: A longitudinal study on dyadic coping and quality of life among couples with a partner suffering from COPD. Journal of Behavioral Medicine, 38, 851-862. https://doi.org/10.1007/s10865-015-9657-y

von Gruenigen, V. E., Huang, H. Q., Gil, K. M., Gibbons, H. E., Monk, B. J., Rose, P. G., .. Wenzel, L. (2010). A comparison of quality-of-life domains and clinical factors in 
ovarian cancer patients: A gynecologic oncology group study. Journal of Pain and Symptom Management, 39, 839-846. https://doi.org/10.1016/j.jpainsymman.2009.09.022

Wei, M., Heppner, P. P., \& Mallinckrodt, B. (2003). Perceived coping as a mediator between attachment and psychological distress: A structural equation modeling approach. Journal of Counseling Psychology, 50, 438-447. https://doi.org/10.1037/0022-0167.50.4.438

Wei, M., Mallinckrodt, B., Russell, D. W., \& Abraham, W. T. (2004). Maladaptive perfectionism as a mediator and moderator between adult attachment and depressive mood. Journal of Counseling Psychology, 51, 201-212. https://doi.org/10.1037/00220167.51.2.201

Wu, L. M., Mohamed, N. E., Winkel, G., \& Diefenbach, M. A. (2013). Patient and spouse illness beliefs and quality of life in prostate cancer patients. Psychology \& Health, 28, 355-368. https://doi.org/10.1080/08870446.2012.722219

Yawn, B. P., Barrette, B. A., \& Wollan, P. C. (2004). Ovarian cancer: The neglected diagnosis. Mayo Clinic Proceedings, 79, 1277-82.

Yost, K. J., \& Eton, D. T. (2005). Combining distribution- and anchor-based approaches to determine minimally important differences: the FACIT experience. Evaluation \& the Health Professions, 28, 172-191. https://doi.org/10.1177/0163278705275340 